Bond University

Research Repository

\title{
Efficacy of ginger (Zingiber officinale) in ameliorating chemotherapy-induced nausea and vomiting and chemotherapy-related outcomes: a systematic review update and meta-analysis
}

Crichton, Megan; Marshall, Skye; Marx, Wolfgang; McCarthy, Alexandra L.; Isenring, Elisabeth

Published in:

Journal of the Academy of Nutrition and Dietetics

DOI:

10.1016/j.jand.2019.06.009

Licence:

CC BY-NC-ND

Link to output in Bond University research repository.

Recommended citation(APA):

Crichton, M., Marshall, S., Marx, W., McCarthy, A. L., \& Isenring, E. (2019). Efficacy of ginger (Zingiber

officinale) in ameliorating chemotherapy-induced nausea and vomiting and chemotherapy-related outcomes: a systematic review update and meta-analysis. Journal of the Academy of Nutrition and Dietetics, 119(12), 20552068. https://doi.org/10.1016/j.jand.2019.06.009

\section{General rights}

Copyright and moral rights for the publications made accessible in the public portal are retained by the authors and/or other copyright owners and it is a condition of accessing publications that users recognise and abide by the legal requirements associated with these rights.

For more information, or if you believe that this document breaches copyright, please contact the Bond University research repository coordinator 
1 Efficacy of ginger (Zingiber officinale) in ameliorating chemotherapy-induced nausea and vomiting and chemotherapy-related outcomes: a systematic review update and meta-

3

4

5

$6 \quad$ Isenring ${ }^{1,6}$

$7 \quad{ }^{1}$ Faculty of Health Sciences and Medicine, Bond University, Gold Coast, Queensland 4226,

8 Australia.

9 analysis

Megan Crichton ${ }^{1}$, Skye Marshall ${ }^{1,2}$, Wolfgang Marx ${ }^{1,3}$, Alexandra L. McCarthy ${ }^{4,5}$, Elizabeth Australia.

${ }^{2}$ Corresponding author. Email: skye marshall@bond.edu.au Telephone: +61 411166230

${ }^{3}$ Deakin University, Food \& Mood Centre, IMPACT Strategic Research Centre, School of Medicine, Barwon Health, Geelong, Victoria 3320, Australia.

${ }^{4}$ School of Nursing, University of Auckland, Auckland 1010, New Zealand.

${ }^{5}$ Division of Cancer Services, Princess Alexandra Hospital, Queensland, Australia.

${ }^{6}$ Department of Nutrition \& Dietetics, Princess Alexandra Hospital, Woolloongabba, Queensland 4102, Australia.

Key words: ginger, nausea, emesis, vomiting, cancer, CINV, systematic review, metaanalysis

Abstract words: 220

Full text words: 5143 
MC led the design, implementation, data analysis and drafting of the manuscript. SM duplicated data extraction and GRADE assessment. WM duplicated risk of bias assessment and GRADE assessment. All authors contributed to study concept and revision of the manuscript. MC completed this work in partial fulfilment of her PhD candidature.

\section{Funding source}

This work was conducted as part of the SPICE trial which was supported by a Cancer Council Queensland Research Project Grant [APP1126733]. Cancer Council Queensland had no input to the study design, implementation, or analysis and reporting of results.

\section{Declaration of conflicting interests}

All authors declare no actual or potential financial or other conflicts of interest.

\section{Acknowledgements}

The authors would like to acknowledge David Honeyman, Librarian at Bond University, for his assistance with designing the search strategy. David Honeymann has given his permission to be named in the acknowledgements.

\section{Research Snapshot}

Research Question: This systematic review update with meta-analyses, in adult cancer patients receiving chemotherapy, aims to determine: what are the effects of ginger supplementation dose and duration on the incidence and severity of chemotherapy-induced nausea and vomiting (CINV) and outcomes related to CINV (e.g. quality of life, fatigue, adverse events), compared to placebo or standard anti-emetic medication? 
50 Key Findings: Eighteen papers were analysed. The likelihood of acute vomiting was reduced 51 by $60 \%$ with ginger supplementation of $\leq 1 \mathrm{~g}$ /day for $>3$-days duration, compared to control 52 groups (OR: 0.4; 95\% CI: $0.17-0.81 ; \mathrm{P}=0.01)$. The likelihood of fatigue was reduced by $80 \%$ 53 with ginger supplementation of any dose for $<3$-days duration (OR: 0.2; 95\% CI: 0.03-0.87; $54 \mathrm{P}=0.03)$. No statistically significant association was found between ginger and likelihood of 55 overall or delayed vomiting, likelihood or severity of nausea, or other outcomes related to 56 CINV. 
Abstract

Background: Ginger has been proposed as an adjuvant treatment for chemotherapy-induced nausea and vomiting (CINV).

Objective: The aim of this systematic review with meta-analyses is to evaluate, in adult cancer patients receiving chemotherapy, the effects of ginger supplementation dose and duration on the incidence, duration, and severity of CINV and outcomes related to CINV (quality of life, fatigue), compared to placebo or standard anti-emetic medication.

Method: Five electronic databases were searched from database inception to April 2018. The quality of evidence was appraised with the Cochrane Risk of Bias tool and GRADE. Data were pooled using Revman.

Results: Eighteen papers were analysed. The likelihood of acute vomiting was reduced by $60 \%$ with ginger supplementation of $\leq 1 \mathrm{~g} /$ day for $>3$-days duration, compared to control groups $(\mathrm{OR}$ : 0.4; 95\% CI: $0.17-0.81 ; \mathrm{P}=0.01 ; \mathrm{n}=3$ studies; $\mathrm{n}=3$ interventions; $\mathrm{n}=301$ participants; $\mathrm{I}^{2}=20 \%$; GRADE level: moderate). The likelihood of fatigue was reduced by $80 \%$ with ginger supplementation of any dose for $<3$-days duration (OR: $0.2 ; 95 \% \mathrm{CI}: 0.03-0.87 ; \mathrm{P}=0.03 ; \mathrm{n}=1$ studies; $n=2$ interventions; $n=219$ participants; $I^{2}=0 \%$; GRADE level: low). No statistically significant association was found between ginger and likelihood of overall or delayed vomiting, likelihood or severity of nausea, or other outcomes related to CINV.

Conclusions: Ginger supplementation might benefit chemotherapy-induced vomiting as well as fatigue. Due to clinical heterogeneity, this systematic review update found no association between ginger and chemotherapy-induced nausea and other CINV-related outcomes. The results of this systematic review and meta-analysis provide a rationale for further research with stronger study designs, adequate sample sizes, standardized ginger products, and validated outcome measures to confirm efficacy of ginger supplementation and optimal dosing regimens. 
Nausea and vomiting are among the most distressing side effects of chemotherapy (CTx). ${ }^{1,2}$

Chemotherapy is a common and effective treatment for cancer; however, chemotherapyinduced nausea and vomiting (CINV) can affect treatment completion as it is known to exacerbate fatigue, anxiety and depression, ${ }^{3}$ as well as decrease quality of life (QoL) and food intake. ${ }^{4-7}$ Consequently, CINV is attributable to $50-60 \%$ of CTx patients experiencing proteinenergy malnutrition as a result of failing to meet nutritional requirements, ${ }^{8}$ which further compromises treatment outcomes. ${ }^{9}$ Good management of $\mathrm{CINV}$ is therefore a priority to optimize treatment efficacy, QoL, and subsequent survival. ${ }^{4-6}$

Anti-emetics to prevent and manage CINV are often prescribed in combination, with the precise regimen tailored to the emetogenicity of the CTx and patient characteristics. ${ }^{10}$ Consensus between the three main clinical guidelines for anti-emetic prescription suggests the administration of a serotonin $\left(5-\mathrm{HT}_{3}\right)$ receptor antagonist and dexamethasone for moderately emetogenic CTx regimens (those with $30-90 \%$ risk of emesis), with the addition of a neurokinin $1\left(\mathrm{NK}_{1}\right)$ receptor antagonist for patients receiving highly emetogenic CTx $(>90 \%$ risk of emesis). ${ }^{11,12}$ Despite pharmacological developments with combination anti-emetics, CINV remains a problematic side effect of CTx. ${ }^{13}$

Ginger (Zingiber officinale) is a traditional remedy for nausea and vomiting in many cultures and has been investigated for use in motion sickness, morning sickness, and post-operative nausea. ${ }^{14}$ More novel is the role of ginger for the prevention and management of CINV. ${ }^{15,16}$ The exact mechanism remains unclear; however, beneficial effects are thought to be due to the effects of gingerol and shogaol compounds on multiple components of CINV pathways. ${ }^{17}$ The most well understood pathway is ginger's antagonistic effect on 5- $\mathrm{HT}_{3}$ receptors. Ginger noncompetitively inhibits $5-\mathrm{HT}_{3}$ receptor activation in humans via binding at a site that is different from other types of 5- $\mathrm{HT}_{3}$ receptor antagonists. ${ }^{18}$ Therefore, synergistic inhibition of $5-\mathrm{HT}_{3}$ 
signalling might occur when ginger is combined with other $5-\mathrm{HT}_{3}$ antagonists, for example ondansetron, a common anti-emetic administered during CTx, suggesting there are additional beneficial effects when ginger is included in anti-emetic regimens. ${ }^{17,19}$ Ginger is also thought to render beneficial effects through its antagonistic effect on muscarinic and histaminergic receptors; its ability to regulate gastric emptying and gastrointestinal motility; and its role in reducing oxidative stress and inflammation. ${ }^{17}$ These multiple pathways may also be linked with other CINV-related symptoms such as fatigue, anxiety, and depression, thereby impacting overall health-related quality of life..$^{3,17,20-22}$

Two systematic reviews, both published in $2013,{ }^{15,16}$ explored the effect of ginger on CINV. The first review identified seven clinical studies that investigated supplemental ginger to treat CINV. ${ }^{15}$ Qualitative examination of included studies was used by Marx et al. ${ }^{15}$ to conclude that the available literature provided mixed support for the use of ginger, not warranting standard recommendations for its use in the clinical setting. The second review identified five doubleblind placebo-controlled randomized trials for inclusion, and meta-analysis determined no significant association between ginger and the control of CINV. ${ }^{16}$ However, both reviews highlighted major limitations in existing research in terms of study design with nonstandardized CTx and anti-emetic regimens, inconsistent and/or inadequate ginger intervention dose and duration, as well as failure to identify or control potentially confounding variables. ${ }^{15,16}$

Due to the inconclusive nature of the previous systematic reviews ${ }^{15,16}$ and more recent publication of clinical trials that examine the effect of ginger on CINV, an updated review of the literature was warranted. The aim of this systematic review update with meta-analyses was to evaluate, in adult cancer patients receiving CTx, the effects of ginger supplementation dose and duration on the incidence and severity of CINV and outcomes related to CINV (quality of life, fatigue), compared to placebo or standard anti-emetic medication. 
Method

132 This systematic review was reported according to the Preferred Reporting Items for Systematic

133 Reviews and Meta-analyses (PRISMA) statement. ${ }^{23}$ The study protocol was registered with the

134 International Prospective Register of Systematic Reviews (PROSPERO number:

135 CRD42017077022).

\section{Search Strategy}

137 PubMed, the Cochrane Library, and CINAHL databases were previously searched by the authors from database inception up until April 2012 to locate both parallel and crossover intervention trials. The findings of that search were reported by Marx et al. ${ }^{15}$ For this update, these databases were searched to locate studies published from April 2012 to April 2018 with the addition of two databases: Embase and Web of Science, which were searched from database inception to 19 September 2017. The search strategy used for this updated review was similarly based upon the following terms: (ginger* OR zingiber officinale) AND (cancer* OR chemotherap*) AND (nausea OR vomit*) AND (randomized controlled trial OR intervention). The search strategy was designed in PubMed using a combination of keywords and controlled vocabulary and translated to other databases with Polyglot ${ }^{24}$ (full search strategy is shown in

147 Online Supplementary Material 1). A snowballing search was also used, whereby reference lists of included studies and previous systematic and narrative reviews were considered to identify additional studies not found in the systematic search strategy up until 4 April 2018. Initial screening of titles and abstracts, as well as full-text screening, were completed by two 151 investigators independently (MC and SM). Disagreement between reviewers was resolved by 152 discussion with a third researcher (WM).

\section{$153 \quad$ Selection of studies}

154 Studies published in any language were included in this review if they provided an intervention 
of ginger supplementation in any form to adult (mean sample age $\geq 18$ years) human participants undergoing CTx for cancer and had used a control of either placebo or anti-emetic medication. Studies were excluded if the population of interest was receiving concurrent radiation therapy, which is a known predictive factor for nausea and vomiting. Studies were included if participants were undergoing other therapies such as surgery, biologics, immunotherapy, and bone marrow transplant therapy. Non-English language studies were also excluded if they could not be translated to English with Google Translate software. ${ }^{25}$ The primary outcomes of interest were CINV incidence (number of participants who reported nausea and/or vomiting of any severity) and nausea severity (measured using any tool). Secondary outcomes were patient QoL, fatigue, anxiety or depression, adverse events (any reported side effects relatable to the intervention), adherence to the intervention, stomach dysrhythmia, tachygastria or bradygastria, nutritional status, dietary intake, health service use, health care costs, and mortality.

Data extraction and review of study quality

Data extraction and individual study quality assessment using the Cochrane Risk of Bias Tool ${ }^{15}$ were carried out independently on all studies, including eligible studies identified in the review by Marx et al., by two authors (MC and [WM or SM]), with disagreements managed by consensus.

The certainty in the body of evidence for each outcome of interest was classified by the Grading of Recommendations, Assessment, Development and Evaluation (GRADE) approach ${ }^{26}$ with the software GRADEpro GDT [GRADEpro Guideline Development Tool, McMaster University, 2015 (developed by Evidence Prime, Inc)]. Four levels of certainty for the estimated effect were utilized by the GRADE assessment: very low (very little confidence), low (limited confidence), moderate (moderately confident), and high (very confident). The 
179 GRADE level of evidence was determined by review and discussion amongst three authors 180 (MC, SM, and WM).

181

182

\section{$\underline{\text { Meta-analysis }}$}

Where two or more interventions reported the same outcome with sufficient incidence or mean and variance data, outcomes for the intervention and control group were pooled by metaanalysis using Revman. ${ }^{27}$ The overall effect size was calculated by combining all the individual studies' outcome data and associated $95 \%$ confidence interval (CI) to calculate an odds ratio or mean difference using the standard random effects method in Revman. The pooled categorical outcomes were reported as odds ratios (OR; ratio of the odds of the outcome in the two groups of interest) with 95\% confidence intervals, using the Mantel-Haenszel test. The inverse variance test pooled continuous outcomes, which were reported as mean differences (MD) where the same tool and scale was used, or the standardized mean difference (SMD) where different measurement scales or tools for the same construct were used. To support clinical interpretation, SMDs were re-expressed into a scale of one of the included measurement tools by multiplying the SMD effect size by the standard deviation of the tool's scale in the total sample. ${ }^{28}$ Heterogeneity was evaluated with the $\mathrm{I}^{2}$ statistic, with $>50 \%$ representative of substantial heterogeneity. ${ }^{29} \mathrm{~A} \mathrm{P}$ value of less than 0.05 was considered the cut off for statistical significance. When meta-analyses included 10 or more studies, a funnel plot was generated by Revman to assess publication bias. If there was a non-significant trend or substantial heterogeneity, sensitivity analyses were undertaken with study factors such as participant characteristics, study design, study quality, and intervention/confounding variables. Additionally, to identify the effect of various dosing regimens, subgroup analyses were performed. Due to conflicting optimal dosing regimens and duration of intervention in the literature, interventions were divided as evenly as possible which generated the daily dosing categories of $\leq 1 \mathrm{~g} />1 \mathrm{~g}$ and duration categories of $\leq 3 />3$ days. Therefore, subgroup analyses 
were undertaken using four groups: 1$)$ short intervention duration ( $\leq 3$ days per CTx cycle) with low dose $(\leq 1 \mathrm{~g} /$ day $) ; 2)$ short intervention duration ( $\leq 3$ days per CTx cycle) with high dose of ginger ( $>1 \mathrm{~g} /$ day); 3) long intervention duration ( $>3$ days per CTx cycle) and low dose of ginger ( $\leq 1 \mathrm{~g} /$ day); 4) long intervention duration ( $>3$ days per CTx cycle) and high dose of ginger ( $>1 \mathrm{~g} /$ day). Additionally, it was hypothesized that ginger could have varying levels of efficacy for anticipatory (prior to CTx), acute ( $\leq 24$ hours post CTx), and delayed ( $>24$ hours post CTx) CINV; therefore, separate analyses were performed for each.

\section{Results}

212 Search results and study quality

The search strategy and study selection process that led to the 18 studies included in this review are displayed in Figure 1. A total of 13 papers were analysed in nine meta-analyses. Figure 2 indicates that risk of bias across all papers was mostly low, with all but four papers included having low risk of bias for at least four of the seven domains (justifications are shown in Online Supplementary Material 2). However, the majority of papers had an unclear or high risk of bias for allocation concealment, mainly due to papers failing to provide a description of concealment procedures. Two papers, ${ }^{30,31}$ which reported nausea outcomes only, had poor study quality due to $>70 \%$ of Cochrane Risk of Bias domains rated as unclear or high risk of bias. Due to the small number of studies in each meta-analysis, publication bias could not be assessed.

Intervention characteristics

Characteristics of the 18 included studies are summarized in Table 1 and shown in detail in

Online Supplementary Material 3. Konmun et al. ${ }^{32}$ and Danwilai et al. ${ }^{33}$ reported different outcomes in each paper; however, they refer to the same study and intervention. One study had two intervention $\operatorname{arms}^{34}$ and another had three intervention arms, ${ }^{35}$ therefore, a total of 20 interventions are referred to in this review. 
229 The active constituents in the ginger interventions were specified in ten interventions.

230 Combined gingerols, zingerone, and shogoals of an unspecified dose were used in three

231 interventions, ${ }^{35}$ gingerols (ranging from 11-16 mg) and shogoal (ranging from 0.92-1.12 mg)

232 were used in four interventions $;^{34,36-38}$ and gingerols (ranging from $0.5-150 \mathrm{mg}$ ) alone were

233 administered in three interventions. ${ }^{32,33,38,39}$ Adherence to the prescribed supplements ranged

234 from $71 \%{ }^{39}$ to $97 \%{ }^{40}$ with no significant difference between intervention and control groups.

\section{$235 \quad$ Study samples}

236 Sample sizes ranged from $n=20^{41}$ to $n=375^{35}$ ( $n=1,652$ total participants; $64 \%$ female) and are

237 reported in detail in Online Supplementary Material 3. Participants in eight studies were 238 administered platinum-based CTx with or without other CTx agents, ${ }^{32,33,36,38,42-45}$ six were 239 administered anthracycline-based $\mathrm{CTx},{ }^{31-33,40,45,46}$ and six studies did not specify the CTx 240 agents administered to participants..$^{30,34,35,37,39,47}$ Six studies reported patients received single241 day CTx treatments, ${ }^{34,36,37,39,47}$ while the remaining studies did not specify days of CTx 242 treatment. Participants in 13 studies were administered a corticosteroid in combination with a 5-HT 3 receptor antagonist; ${ }^{31-33,35-37,40-44,46,47}$ and seven were administered additional antiemetics. $^{31,32,36,40,43,44,47}$

\section{Efficacy of ginger supplementation for nausea and vomiting outcomes}

246

247

Table 2 contains all meta-analyses conducted for primary and secondary outcomes. Forest plots for all non-significant meta-analyses are shown in Online Supplementary Material 4 and justifications for the all GRADE levels are shown in Online Supplementary Material 5. In terms of primary outcomes, the only significant finding was for duration of $>3$ days of supplementation with doses $\leq 1 \mathrm{~g}$ /day which reduced the likelihood of acute vomiting by $60 \%$ (Figure 3). Results regarding anticipatory CINV were unable to be pooled due to lack of studies that reported this outcome. Only one study reported results related to anticipatory nausea, 
finding no significant difference in likelihood of anticipatory nausea between control and intervention groups. ${ }^{36}$ However, it is unclear whether participants in this study were consuming the intervention at the time of anticipatory nausea being measured (at day 21 or 28 prior to commencing subsequent CTx cycles). Only one study reported results related to anticipatory vomiting, which found significantly higher incidence among the control group compared to those who received ginger supplementation ( $\mathrm{IG} n=0.5 / 40$ (SD 0.3); $C G \mathrm{n}=1.5 / 40$ (SD 5.9); $\mathrm{P}=0.04) .{ }^{37}$

In terms of secondary outcomes, three of the four studies ${ }^{32,38,39}$ that reported data on QoL found a statistically significant difference in QoL scores between control and intervention groups at the end of the study period, favouring ginger intervention over control, however, no significant association was found with meta-analysis (Table 2). The only significant findings in regards to fatigue were identified with sensitivity analysis, which found ginger supplementation of any dose for $\leq 3$-days reduced likelihood of fatigue by $80 \%$ (Figure 4 ). No studies reported outcomes on anxiety and depression; stomach dysrhythmia, tachygastria or bradygastria; nutrition status; dietary intake; health service use; or health care costs.

Seven studies reported on events believed to be directly attributable to the intervention. ${ }^{33,35-}$ ${ }^{39,43}$ Of these, three provided adequate data for meta-analysis, finding ginger supplementation of any dose for any duration significantly increased likelihood of any gastrointestinal, flushing, rash-related, or unspecified adverse event (Figure 5). Reported gastrointestinal symptoms potentially relatable to the study intervention included dry mouth, heartburn, reflux, constipation and diarrhoea; however, due to insufficient data for meta-analysis, only the results on heartburn were able to be pooled, finding no association between ginger supplementation of any dose for any duration and likelihood of heartburn. Studies reported no significant differences between adverse events not directly attributable to the intervention, which included neutropenia and other unspecified biochemical markers, restlessness, headache, and heart 
palpitations. Two studies reported incidence of mortality, with no statistically significant difference reported between intervention and control groups. ${ }^{44,45}$

\section{Discussion}

This systematic review update with meta-analyses provides the most current and comprehensive meta-analysis to date exploring the use of ginger for CTx patients. Consistent with conclusions made in the previous two systematic reviews on the topic, ${ }^{48,49}$ ginger supplementation in conjunction with standard anti-emetic care could be beneficial for CTxinduced vomiting and CINV-related outcomes. Although there is low to very low certainty in the estimated effect size, ginger supplementation is suggested to have large beneficial effects on likelihood of acute vomiting, as well as small but significant improvements on fatigue among individuals receiving CTx. Findings remain inconclusive as to whether ginger benefits delayed CTx-induced vomiting, CTx-induced nausea, and QoL.

Despite most papers that reported on nausea incidence favouring ginger supplementation over comparator interventions, ${ }^{30,38,42,45,46,50}$ the pooled estimate found no association with no statistical heterogeneity $\left(\mathrm{I}^{2}=0 \%\right)$, therefore there is some confidence in this finding. However, meta-analyses results in regards to likelihood of nausea appear to be skewed by one larger study. ${ }^{36}$ Therefore, ginger supplementation for improvements in nausea incidence cannot yet be disregarded as a treatment option due to the substantially large clinical heterogeneity between studies.

The non-significant finding which favoured control for nausea severity, particularly for delayed nausea severity when administered for $\leq 3$-days, was driven by only two studies; ${ }^{34,42}$ which were the only studies included in the model after sensitivity analysis. Although there was no statistical heterogeneity, there was substantial clinical, as well as only a small number of studies included which used a range of un-validated outcome evaluation questionnaires. 
This review found low confidence that supplementation of $\leq 1 \mathrm{~g} /$ day of ginger for $>3$-days, starting on Day 1 of CTx reduces likelihood of vomiting by $20-70 \%$; where other dosing regimens were found to have no significant association. Uncertainty in regards to the ideal ginger dosage is likely due to the clinical heterogeneity. Ginger supplementation of $\leq 1 \mathrm{~g} /$ day for $>3$-days potentially reduces likelihood of overall vomiting by $50 \%$ (OR: 0.5 ; $95 \%$ CI: 0.21 1.13 ) and delayed vomiting by $70 \%$ (OR: $0.3 ; 95 \%$ CI: $0.10-1.13$ ); however, the results were not significant at $\mathrm{p}<0.05$, likely attributable to significant clinical heterogeneity among included studies. The only statistically significant finding that ginger administration for $>3$ days benefited acute vomiting is conflicting, as only ginger consumed prior to and within the first 24-hours is applicable to acute symptoms (i.e. symptoms within the first 24 hours). This unlikely finding is further evidence of the impact of clinical heterogeneity leading to decreased confidence in the results, suggesting that until further well-designed studies with homogenous or well-controlled samples are available, all results should be considered with caution, including those with no statistical heterogeneity.

In terms of dosage frequency, research suggests ginger should be distributed at least four times across the day rather than once or twice daily due to ginger's short elimination half-life of $\leq 2$ hours. ${ }^{51}$ However, most studies in this review administered ginger only once or twice per day, which suggests the effects of ginger may be underestimated. Furthermore, the delivery method of ginger administered may also affect the half-life. Although the half-life of ginger is short, production of ginger-induced intestinal microbiota metabolites may be a suggested mechanism for reducing activation of CINV pathways. ${ }^{17,52,53}$

This was the first systematic review to explore additional CINV-related outcomes, finding that ginger resulted in beneficial effects outside of the direct CINV pathways. A decrease in fatigue was seen with ginger administration, a common and problematic symptom otherwise difficult to treat and manage. ${ }^{54}$ The decrease may be due to ginger's proposed anti-inflammatory effects, 
as inflammation is a pathway for fatigue, ${ }^{17,20-22}$ however, none of the included studies examined inflammatory markers. Further studies are needed to build confidence in the body of evidence for the effect of ginger on other CINV-related outcomes.

Adherence to the intervention was found to be high overall and no different to placebo groups, implying that ginger supplementation as an additional medication is feasible and does not impose any additional factors which might prevent adherence. Although this review found ginger did not cause serious adverse events and therefore could be considered safe, it should be noted that there was poor reporting of adverse events in most studies as well as a failure to distinguish events reasonably relatable to the intervention. Although statistically significant results were obtained to suggest likelihood of adverse events is higher among individuals administered ginger, the adverse events were mild. Meta-analysis suggested that the likelihood of heartburn, a commonly reported side effect of ginger, did not differ between the study groups. Mortality, arguably the most serious adverse event, also did not appear to differ between study groups in the limited number of studies that reported this event.

\section{Limitations in the literature}

Although a substantial number of new studies were identified in this systematic review update, the included studies are clinically heterogeneous, which decreases confidence in the results and contributes to the large number analyses which had insufficient evidence to reject the null hypothesis. Sources of clinical heterogeneity were varying CTx and anti-emetic regimens as well as cancer types and participant samples. In addition, substantial clinical heterogeneity was introduced through lack of reporting of, or large variations across studies in ginger interventions; specifically, the composition of active constituents, dosing frequencies and delivery methods. Sensitivity analysis was able to be explored for study quality and overall ginger dosing regimens, but other sources of clinical heterogeneity could not be accounted for 
and publication bias was not able to be assessed. Furthermore, this review was limited by the small number of available studies that reported the outcomes of interest.

\section{$354 \quad$ Directions for future research}

The results of this systematic review and meta-analysis provide a rationale for further research such as that which is currently underway. ${ }^{55}$ In line with recommendations from the previous systematic review, ${ }^{48}$ additional randomized controlled trials with adequate sample sizes, standardized ginger products, use of validated outcome measures, and full reporting of data would better inform the evidence regarding the type of ginger supplement that should be recommended and the most effective dosing schedule. Study designs should also measure adverse events potentially relatable to the ginger intervention to determine the safety of the intervention. Other CTx-related outcomes that are associated with CINV need to be assessed, including anxiety and depression, stomach dysrhythmia, and tachygastria or bradygastria. Other factors that need evaluation related to overall health include nutrition status, dietary intake, health service use, and health care costs. Patients receiving multi-day CTx regimens should be included in studies before recommending ginger products to all patients receiving CTx. The potential mechanisms of action of ginger having beneficial effects on CINV pathways also need investigation.

\section{Conclusion}

Ginger supplementation can potentially benefit CTx-induced vomiting as well as fatigue. Due to clinical heterogeneity, this systematic review update with meta-analyses found no association between ginger and CTx-induced nausea or other CINV-related outcomes. The results of this systematic review and meta-analysis provide a rationale for further research using stronger study designs, adequate sample sizes, standardized ginger products, and validated outcome measures to confirm efficacy of ginger supplementation and optimal dosing regimens. 


\section{References}

378 1. Ferlay J, Soerjomataram I, Dikshit R, et al. Cancer incidence and mortality worldwide: 379 Sources, methods and major patterns in GLOBOCAN 2012. Int $J$ Cancer. 2015;136:E359-E386.

2. World Health Organisation. Diet, Nutrition and the Prevention of Chronic Diseases. Geneva: World Health Organisation;2003.

3. Breen SJ, Baravelli CM, Schofield PE, Jefford M, Yates PM, Aranda SK. Is symptom burden a predictor of anxiety and depression in patients with cancer about to commence chemotherapy? Med J Aust. 2009;190(7):S99-S104.

4. Isenring E, Cross G, Kellett E, Koczwara B, Daniels L. Nutritional status and information needs of medical oncology patients receiving treatment at an Australian public hospital. Nutr Cancer. 2010;62(2):220-228.

5. Segura A, Pardo J, Jara C, et al. An epidemiological evaluation of the prevalence of malnutrition in Spanish patients with locally advanced or metastatic cancer. Clin Nutr. 2005;24(5):801-814.

6. Read JA, Crockett N, Volker DH, et al. Nutritional assessment in cancer: comparing the Mini-Nutritional Assessment (MNA) with the scored Patient-Generated Subjective Global Assessment (PGSGA). Nutr Cancer. 2005;53(1):51-56.

7. Marx W, Kiss N, McCarthy A, McKavanagh D, Isenring E. Chemotherapy-Induced Nausea and Vomiting: A Narrative Review to Inform Dietetics Practice. JAND. 2016;116(5):819-827.

8. Segura A, Pardo J, Jara C, et al. An epidemiological evaluation of the prevalence of malnutrition in Spanish patients with locally advanced or metastatic cancer. Clin Nutr. 2005;24(5):801-814.

9. Marshall S. Why is the skeleton still in the hospital closet? A look at the complex aetiology of malnutrition and its implications for the nutrition care team. J Nutr Health Aging. 2018;22:26-29.

10. Hesketh PJ, Kris MG, Basch E, et al. Antiemetics: American Society of Clinical Oncology Clinical Practice Guideline Update. J Clin Oncol 2017;35(28):3240-3261.

11. Roila F, Molassiotis A, Herrstedt J, et al. 2016 MASCC and ESMO guideline update for the prevention of chemotherapy- and radiotherapy-induced nausea and vomiting and of nausea and vomiting in advanced cancer patients. Ann Oncol. 2016;27(5):v119-v133.

12. National Comprehensive Cancer Network. National Comprehensive Cancer Network (NCCN) Clinical Practice Guidelines in Oncology. 2018. 
13. Bloechl-Daum B, Deuson RR, Mavros P, Hansen M, Herrstedt J. Delayed nausea and vomiting continue to reduce patients' quality of life after highly and moderately emetogenic chemotherapy despite antiemetic treatment. $J$ Clin Oncol 2006;24(27):4472-4478.

14. Ali BH, Blunden G, Tanira MO, Nemmar A. Some phytochemical, pharmacological and toxicological properties of ginger (Zingiber officinale Roscoe): A review of recent research. Food Cheml Toxicol. 2008;46:409-420.

15. Marx WM, Teleni L, McCarthy AL, et al. Ginger (Zingiber officinale) and chemotherapy-induced nausea and vomiting: a systematic literature review. Nutr Rev. 2013;71(4):245-254.

16. Lee J, Heeyoung O. Ginger as an Antiemetic Modality for Chemotherapy-Induced Nausea and Vomiting: A Systematic Review and Meta-Analysis. Oncol Nurs Forum. 2013;40(2):163-170.

17. Marx W, Ried K, McCarthy AL, et al. GingerMechanism of action in chemotherapyinduced nausea and vomiting: A review. Crit Rev Food Sci Nutr. 2017;57(1):141-146.

18. Walstab J, Kruger D, Stark T, et al. Ginger and its pungent constituents noncompetitively inhibit activation of human recombinant and native 5-HT3 receptors of enteric neurons. Neurogastroenterol Motil. 2013;25(5):439-447, e302.

19. Walstab J, Kruger D, Stark T, et al. Ginger and its pungent constituents noncompetitively inhibit activation of human recombinant and native 5-HT3 receptors of enteric neurons. Neurogastroenterol Motil. 2013;25(5):439-447.

20. Mashhadi NS, Ghiasvand R, Askari G, Hariri M, Darvishi L, Mofid MR. Anti-oxidative and anti-inflammatory effects of ginger in health and physical activity: Review of current evidence. Int J Prev Med. 2013;4(1):S36-S42.

21. Louati K, Berenbaum F. Fatigue in chronic inflammation - a link to pain pathways. Arthritis Res Ther. 2015;17(254).

22. Meyers CA, Albitar M, Estey E. Cognitive impairment, fatigue, and cytokine levels in patients with acute myelogenous leukemia or myelodysplastic syndrome. Cancer. 2005; 104:788-793.

23. Moher D, Liberati A, Tetzlaff J, Altman DG, Group P. Preferred reporting items for systematic reviews and meta-analyses: the PRISMA statement. Int $J$ Surg. 2010;8(5):336-341.

24. Center for Research in Evidence-Based Practice Systematic Review Accelerator (CREBP-SRA). Polyglot Search Translator. http://crebp-sra.com/ - / Published 2017. Accessed July 14, 2018.

25. Google. Google Translate. https://translate.google.com.au/. Published 2017. Accessed July 29, 2018. 
26. Guyatt GH, Oxman AD, Schunemann HJ, Tugwell P, Knottnerus A. GRADE guidelines: a new series of articles in the Journal of Clinical Epidemiology. J Clin Epidemiol. 2011;64(4):380-382.

27. Review Manager 5 [computer program]. Version 5.3: Cochrane Informatics \& Knowledge Management Department; Published 2014.

28. Higgins J, Green S. Cochrane Handbook for Systematic Reviews of Interventions: 17.8.2: Study summaries using more than one patient-reported outcome. 2011.

29. Ryan, R. Heterogeneity and subgroup analyses in Cochrane Consumers and Communication Group reviews: planning the analysis at protocol stage. Cochrane Consumers and Communication Review Group; 2016.

30. Alparslan GB, Ozkarman A, Eskin N, et al. Effect of Ginger on Chemotherapy-Induced Nausea and/or Vomiting in Cancer Patients. J Aust Tradit-Med Soc. 2012;18(1):15-18.

31. Arslan M, Ozdemir L. Oral intake of ginger for chemotherapy-induced nausea and vomiting among women with breast cancer. Clin J Oncol Nurs. 2015;19(5):E92-97.

32. Konmun J, Danwilai K, Ngamphaiboon N, Sripanidkulchai B, Sookprasert A, Subongkot S. A phase II randomized double-blind placebo-controlled study of 6gingerol as an anti-emetic in solid tumor patients receiving moderately to highly emetogenic chemotherapy. Med Oncol. 2017;34(4):69.

33. Danwilai K, Konmun J, Sripanidkulchai BO, Subongkot S. Antioxidant activity of ginger extract as a daily supplement in cancer patients receiving adjuvant chemotherapy: A pilot study. Cancer Manag Res. 2017;9:11-18.

34. Zick SM, Ruffin MT, Lee J, et al. Phase II trial of encapsulated ginger as a treatment for chemotherapy-induced nausea and vomiting. Support Care Cancer. 2009;17(5):563-572.

35. Ryan JL, Heckler CE, Roscoe JA, et al. Ginger (Zingiber officinale) reduces acute chemotherapy-induced nausea: a URCC CCOP study of 576 patients. Support Care Cancer. 2012;20(7):1479-1489.

36. Bossi P, Cortinovis D, Fatigoni S, et al. A randomized, double-blind, placebocontrolled, multicenter study of a ginger extract in the management of chemotherapyinduced nausea and vomiting (CINV) in patients receiving high dose cisplatin. Ann Oncol. 2017;28(10):2547-2551.

37. Yekta ZP, Ebrahimi SM, Hosseini M, et al. Ginger as a miracle against chemotherapyinduced vomiting. Iran J Nurs Midwifery Res. 2012;17(5):325-329.

38. Li X, Qin Y, Liu W, Zhou X, Li Y, Wang L. Efficacy of Ginger in Ameliorating Acute and Delayed Chemotherapy-Induced Nausea and Vomiting Among Patients With Lung Cancer Receiving Cisplatin-Based Regimens: A Randomized Controlled Trial. Integr Cancer Ther. 2018;17(3):747-754. 
39. Marx W, McCarthy AL, Ried K, et al. The Effect of a Standardized Ginger Extract on Chemotherapy-Induced Nausea-Related Quality of Life in Patients Undergoing Moderately or Highly Emetogenic Chemotherapy: A Double Blind, Randomized, Placebo Controlled Trial. Nutrients. 2017;9(8).

40. Thamlikitkul L, Srimuninnimit V, Akewanlop C, et al. Efficacy of ginger for prophylaxis of chemotherapy-induced nausea and vomiting in breast cancer patients receiving adriamycin-cyclophosphamide regimen: a randomized, double-blind, placebo-controlled, crossover study. Support Care Cancer. 2017;25(2):459-464.

41. Muthia R, Wahyu W, Dachriyanus. Effect Of Ginger Infusion On Chemotherapy Induced Nausea And Vomiting In Breast Cancer Patients. J Biol Agr Healthcare. 2013;3(13):42-46.

42. Fahimi F, Khodadad K, Amini S, et al. Evaluating the Effect of Zingiber Officinalis on Nausea and Vomiting in Patients Receiving Cisplatin Based Regimens. Iran J Pharm Res. 2011;10(2):379-384.

43. Manusirivithaya S, Sripramote M, Tangjitgamol S, et al. Antiemetic effect of ginger in gynecologic oncology patients receiving cisplatin. Int $J$ Gynecol Cancer. 2004;14(6):1063-1069.

44. Montazeri AS, Raei M, Ghanbari A, Dadgari A, Montazeri AS, Hamidzadeh A. Effect of herbal therapy to intensity chemotherapy-induced nausea and vomiting in cancer patients. Iran Red Crescent Med J. 2013;15(2):101-106.

45. Shokri F, Gharebaghi P, Esfahani A, et al. Comparison of the complications of platinum-based adjuvant chemotherapy with and without ginger in a pilot study on ovarian cancer patients. Int J Womens Health Reproduction Sci. 2017;5(4):324-331.

46. Panahi Y, Saadat A, Sahebkar A, Hashemian F, Taghikhani M, Abolhasani E. Effect of ginger on acute and delayed chemotherapy-induced nausea and vomiting: a pilot, randomized, open-label clinical trial. Integr Cancer Ther. 2012;11(3):204-211.

47. Sanaati F, Najafi S, Kashaninia Z, Sadeghi M. Effect of Ginger and Chamomile on Nausea and Vomiting Caused by Chemotherapy in Iranian Women with Breast Cancer. Asian Pac J Cancer Prev. 2016;17(8):4125-4129.

48. Marx WM, Teleni L, McCarthy AL, et al. Ginger (Zingiber officinale) and chemotherapy-induced nausea and vomiting: a systematic literature review. Nutrition Rev. 2013;71(4):245-254.

49. Lee J, Oh H. Ginger as an antiemetic modality for chemotherapy-induced nausea and vomiting: a systematic review and meta-analysis. Oncol Nurs Forum. 2013;40(2):163170.

50. Konmun J, Danwilai K, Ngamphaiboon N, Sookprasert A, Sirachainan E, Subongkot S. A phase II randomized, double-blind placebo-controlled trial of an antiemetic, 6gingerol in solid tumor patients receiving moderately to highly emetogenic adjuvant chemotherapy. J Clin Oncol. 2014;32(15):1. 
51. Zick SM, Djuric Z, Ruffin MT, et al. Pharmacokinetics of 6-, 8-, 10-Gingerols and 6Shogaol and conjugate metabolites in healthy human subjects. Cancer Epidemiol Biomarkers Prev. 2008;17(8):1930-1936.

52. Reigstad CS, Salmonson CE, Rainey JF. Gut microbes promote colonic serotonin production through an effect of short-chain fatty acids on enterochromaffin cells. FASEB. 2015;29(4):1395-1403.

53. Yano JM, Yu K, Donaldson GP. Indigenous bacteria from the gut microbiota regulate host serotonin biosynthesis. Cell. 2015;161(2):264-276.

54. Marx W, Teleni L, Opie RS, et al. Efficacy and effectiveness of carnitine supplementation for cancer-related fatigue: A systematic literature review and metaanalysis. Nutrients. 2017;9:1224.

55. Marx W, McCarthy A, Marshall S, et al. Supplemental prophylactic intervention for chemotherapy-induced nausea and emesis (SPICE) trial: Protocol for a multicentre double-blind placebo-controlled randomised trial. Nutr Diet. 2018. 
590 Table 1. Summary of intervention characteristics and study samples of the 18 included studies in this systematic review with meta-analyses aiming 591 to evaluate, in adult cancer patients receiving chemotherapy, the effects of ginger supplementation dose and duration on the incidence and severity 592 of chemotherapy-induced nausea and vomiting and outcomes related to chemotherapy-induced nausea and vomiting (quality of life, fatigue).

\begin{tabular}{|c|c|c|c|c|c|c|c|c|c|}
\hline Citation & Country & Study Design & $\begin{array}{l}\text { Sample } \\
\text { Size }\end{array}$ & $\begin{array}{l}\text { CTX } \\
\text { emetogenicity }\end{array}$ & $\begin{array}{l}\text { Anti-emetic } \\
\text { Use (Yes/No) }\end{array}$ & Intervention & Intervention dose & Intervention duration & Comparator \\
\hline $\begin{array}{l}\text { Alparslan } \\
2012^{30}\end{array}$ & Turkey & Non-RCT & $\mathrm{N}=45$ & Not specified & Not specified & $\begin{array}{l}\text { Tablet } \\
\text { (ginger type unspecified) }\end{array}$ & $\begin{array}{l}1.6 \mathrm{~g} / \mathrm{d} \\
(2 \times 0.4 \mathrm{~g} \mathrm{BD})\end{array}$ & Entire treatment duration & IV antiemetic \\
\hline $\begin{array}{l}\text { Arslan } \\
2015^{31}\end{array}$ & Turkey & RCT & $\mathrm{N}=60$ & Not specified & Yes & $\begin{array}{l}\text { Sachet } \\
\text { (powdered ginger root on } \\
\text { yoghurt) }\end{array}$ & $\begin{array}{l}1 \mathrm{~g} / \mathrm{d} \\
(0.5 \mathrm{~g} \mathrm{BD})\end{array}$ & $\begin{array}{l}3 \text { days; from } 30 \text { mins } \\
\text { before Cycle } 1 \text { Day } 1\end{array}$ & Standard care \\
\hline $\begin{array}{l}\text { Bossi } \\
2017^{36}\end{array}$ & Italy & $\begin{array}{l}\text { Double blind placebo } \\
\text { RCT }\end{array}$ & $\mathrm{N}=251$ & High & Yes & $\begin{array}{l}\text { Capsule } \\
\text { (liquid ginger root extract) }\end{array}$ & $\begin{array}{l}0.16 \mathrm{~g} / \mathrm{d} \\
(2 \times 0.04 \mathrm{~g} \mathrm{BD})\end{array}$ & $\begin{array}{l}42-56 \text { days; from Day } 2 \text { of } \\
\text { each Cycle }\end{array}$ & Placebo \\
\hline $\begin{array}{l}\text { Danwilai } \\
2017^{33}\end{array}$ & Thailand & $\begin{array}{l}\text { Pilot double blind } \\
\text { placebo RCT }\end{array}$ & $\mathrm{N}=50$ & $\begin{array}{l}\text { Moderate or } \\
\text { high }\end{array}$ & Yes & $\begin{array}{l}\text { Capsule } \\
\text { (powdered ginger root } \\
\text { extract) }\end{array}$ & $\begin{array}{l}0.02 \mathrm{~g} / \mathrm{d} \\
(2 \times 0.005 \mathrm{~g} \mathrm{BD})\end{array}$ & $\begin{array}{l}\text { From } 3 \text { days prior to Cycle } \\
1 \text { Day } 1 \text { through to Cycle } 4\end{array}$ & Placebo \\
\hline $\begin{array}{l}\text { Fahimi } \\
2011^{42}\end{array}$ & Iran & $\begin{array}{l}\text { Double blind } \\
\text { crossover placebo } \\
\text { RCT }\end{array}$ & $\mathrm{N}=50$ & Not specified & Yes & $\begin{array}{l}\text { Capsule } \\
\text { (powdered ginger root } \\
\text { extract) }\end{array}$ & $\begin{array}{l}1 \mathrm{~g} \\
(2 \times 0.25 \mathrm{~g} \mathrm{BD})\end{array}$ & $\begin{array}{l}3 \text { days; from Cycle } 1 \text { Day } \\
1 \text {, then a 3-week washout } \\
\text { period }\end{array}$ & Placebo \\
\hline $\begin{array}{l}\text { Konmun } \\
2017^{32}\end{array}$ & Thaliand & $\begin{array}{l}\text { Double blind placebo } \\
\text { RCT }\end{array}$ & $\mathrm{N}=88$ & $\begin{array}{l}\text { Moderate or } \\
\text { high }\end{array}$ & Yes & $\begin{array}{l}\text { Capsule } \\
\text { (type unspecified) }\end{array}$ & $\begin{array}{l}0.02 \mathrm{~g} / \mathrm{d} \\
(2 \times 0.005 \mathrm{~g} \mathrm{BD})\end{array}$ & $\begin{array}{l}\text { From } 3 \text { days prior to CTx } \\
\text { Day } 1 \text { for at least } 3 \text { Cycles }\end{array}$ & Placebo \\
\hline $\begin{array}{l}\mathrm{Li} \\
2018^{38}\end{array}$ & China & $\begin{array}{l}\text { Double blind placebo } \\
\text { RCT }\end{array}$ & $\mathrm{N}=146$ & $\begin{array}{l}\text { Moderate or } \\
\text { high }\end{array}$ & Yes & $\begin{array}{l}\text { Capsule } \\
\text { (powdered ginger root } \\
\text { extract) }\end{array}$ & $\begin{array}{l}0.5 \mathrm{~g} / \mathrm{d} \\
(2 \times 0.25 \mathrm{~g} \mathrm{BD})\end{array}$ & 5 days; from CTx Day 1 & Placebo \\
\hline $\begin{array}{l}\text { Manusirivithaya } \\
2004^{43}\end{array}$ & Thaliand & $\begin{array}{l}\text { Double blind } \\
\text { crossover RCT }\end{array}$ & $\mathrm{N}=48$ & High & Yes & $\begin{array}{l}\text { Capsule } \\
\text { (powdered ginger root } \\
\text { extract) }\end{array}$ & $\begin{array}{l}\mathrm{g} \\
(0.25 \mathrm{~g} \text { QID) }\end{array}$ & $\begin{array}{l}5 \text { days; then a 3-4 week } \\
\text { washout period }\end{array}$ & Placebo \\
\hline Marx & Australia & $\begin{array}{l}\text { Double blind placebo } \\
\text { RCT }\end{array}$ & $\mathrm{N}=51$ & Not specified & Yes & Capsule & $1.2 \mathrm{~g}$ & 5 days; from CTx Day 1 & Placebo \\
\hline
\end{tabular}




\begin{tabular}{|c|c|c|c|c|c|c|c|c|c|}
\hline $2017^{39}$ & & & & & & $\begin{array}{l}\text { (powdered ginger root } \\
\text { extract) }\end{array}$ & (0.3g QID) & & \\
\hline $\begin{array}{l}\text { Montazeri } \\
2013^{44}\end{array}$ & Iran & $\begin{array}{l}\text { Crossover double } \\
\text { blind placebo RCT }\end{array}$ & $\mathrm{N}=44$ & Not specified & Yes & $\begin{array}{l}\text { Capsule } \\
\text { (powdered ginger root } \\
\text { extract) }\end{array}$ & $\begin{array}{l}1 \mathrm{~g} / \mathrm{d} \\
(2 \times 0.25 \mathrm{~g} \mathrm{BD})\end{array}$ & $\begin{array}{l}\text { For one Cycle (at least } 28 \\
\text { days) before crossing over }\end{array}$ & Placebo \\
\hline $\begin{array}{l}\text { Muthia } \\
2013^{41}\end{array}$ & Indonesia & Control time series & $\mathrm{N}=20$ & Not specified & Yes & $\begin{array}{l}\text { Drink } \\
\text { (type unspecified) }\end{array}$ & $\begin{array}{l}\text { 1 serve } \\
\text { (QID) }\end{array}$ & Not specified & Standard care \\
\hline $\begin{array}{l}\text { Panahi } \\
201246\end{array}$ & Iran & Open pilot RCT & $\mathrm{N}=100$ & $\begin{array}{l}\text { Moderate or } \\
\text { high }\end{array}$ & Yes & $\begin{array}{l}\text { Capsule } \\
\text { (powdered ginger root } \\
\text { extract) }\end{array}$ & $\begin{array}{l}1.5 \mathrm{~g} \\
(0.5 \mathrm{~g} \text { TID) }\end{array}$ & 5 days; from CTx Day 1 & Placebo \\
\hline \multirow[t]{3}{*}{$\begin{array}{l}\text { Ryan } \\
2012^{35}\end{array}$} & USA & $\begin{array}{l}\text { Double blind placebo } \\
\text { RCT }\end{array}$ & $\mathrm{N}=371$ & Any & Yes & $\begin{array}{l}\text { Capsule } \\
\text { (liquid ginger root extract) }\end{array}$ & $\begin{array}{l}0.5 \mathrm{~g} / \mathrm{d} \\
(2 \times 0.25 \mathrm{~g})\end{array}$ & $\begin{array}{l}6 \text { days; from } 3 \text { days before } \\
\text { CTx Day } 1\end{array}$ & Placebo \\
\hline & & & $\mathrm{N}=375$ & Any & Yes & $\begin{array}{l}\text { Capsule } \\
\text { (liquid ginger root extract) }\end{array}$ & $\begin{array}{l}1 \mathrm{~g} / \mathrm{d} \\
(4 \times 0.25 \mathrm{~g})\end{array}$ & $\begin{array}{l}6 \text { days; from } 3 \text { days before } \\
\text { CTx Day } 1\end{array}$ & Placebo \\
\hline & & & $\mathrm{N}=375$ & Any & Yes & $\begin{array}{l}\text { Capsule } \\
\text { (liquid ginger root extract) }\end{array}$ & $\begin{array}{l}1.5 \mathrm{~g} / \mathrm{d} \\
(6 \mathrm{x} 0.25 \mathrm{~g})\end{array}$ & $\begin{array}{l}6 \text { days; from } 3 \text { days before } \\
\text { CTx Day } 1\end{array}$ & Placebo \\
\hline $\begin{array}{l}\text { Sanaati } \\
2016^{47}\end{array}$ & Iran & Double blind RCT & $\mathrm{N}=43$ & Not specified & Yes & $\begin{array}{l}\text { Capsule } \\
\text { (powdered ginger root } \\
\text { extract) }\end{array}$ & $\begin{array}{l}1 \mathrm{~g} / \mathrm{d} \\
(0.5 \mathrm{~g} \mathrm{BD})\end{array}$ & $\begin{array}{l}10 \text { days; from } 5 \text { days } \\
\text { before CTx Day } 1\end{array}$ & Standard care \\
\hline $\begin{array}{l}\text { Shokri } \\
2017^{45}\end{array}$ & Iran & $\begin{array}{l}\text { Double blind placebo } \\
\text { RCT }\end{array}$ & $\mathrm{N}=49$ & Not specified & Not specified & $\begin{array}{l}\text { Capsule } \\
\text { (type unspecified) }\end{array}$ & $\begin{array}{l}2 \mathrm{~g} / \mathrm{d} \\
(1 \mathrm{~g} \mathrm{BD})\end{array}$ & For 6 Cycles & Placebo \\
\hline $\begin{array}{l}\text { Thamlikitkul } \\
2017^{40}\end{array}$ & Thailand & $\begin{array}{l}\text { Double blind } \\
\text { crossover placebo } \\
\text { RCT }\end{array}$ & $\mathrm{N}=34$ & High & Yes & $\begin{array}{l}\text { Capsule } \\
\text { (powdered ginger root } \\
\text { extract) }\end{array}$ & $\begin{array}{l}1 \mathrm{~g} \\
(0.5 \mathrm{~g} \mathrm{BD})\end{array}$ & 5 days; from CTx Day 1 & Placebo \\
\hline $\begin{array}{l}\text { Yekta } \\
2012^{37}\end{array}$ & Iran & $\begin{array}{l}\text { Double blind placebo } \\
\text { RCT }\end{array}$ & $\mathrm{N}=98$ & Any & Yes & $\begin{array}{l}\text { Capsule } \\
\text { (powdered ginger root } \\
\text { extract) }\end{array}$ & $\begin{array}{l}1 \mathrm{~g} \\
(0.25 \mathrm{~g} \text { QID) }\end{array}$ & $\begin{array}{l}6 \text { days; from } 3 \text { days before } \\
\text { CTx Day } 1\end{array}$ & Placebo \\
\hline
\end{tabular}




\begin{tabular}{|c|c|c|c|c|c|c|c|c|c|}
\hline \multirow[t]{2}{*}{$\begin{array}{l}\text { Zick } \\
2009^{34}\end{array}$} & \multirow[t]{2}{*}{ USA } & \multirow[t]{2}{*}{$\begin{array}{l}\text { Double blind placebo } \\
\text { RCT }\end{array}$} & $\mathrm{N}=110$ & Any & Yes & $\begin{array}{l}\text { Capsule } \\
\text { (powdered ginger root } \\
\text { extract) }\end{array}$ & $\begin{array}{l}\mathrm{g} / \mathrm{d} \\
(4 \times 0.25 \mathrm{~g})\end{array}$ & 3 days; from CTx Day 1 & Placebo \\
\hline & & & $\mathrm{N}=109$ & Any & Yes & $\begin{array}{l}\text { Capsule } \\
\text { (powdered ginger root } \\
\text { extract) }\end{array}$ & $\begin{array}{l}2 \mathrm{~g} / \mathrm{d} \\
(8 \mathrm{x} 0.25 \mathrm{~g})\end{array}$ & 3 days; from CTx Day 1 & Placebo \\
\hline
\end{tabular}


Table 2. Results from meta-analyses conducted to evaluate, in adult cancer patients receiving chemotherapy, the effects of ginger supplementation dose and duration on the incidence and severity of chemotherapy-induced nausea and vomiting and outcomes related to chemotherapy-induced nausea and vomiting (quality of life, fatigue).

\begin{tabular}{|c|c|c|c|c|c|c|}
\hline Outcome & $\begin{array}{l}\text { Pooled } \\
\text { estimate }\end{array}$ & $\begin{array}{l}\text { Significance } \\
\text { of pooled } \\
\text { estimate }\end{array}$ & $\begin{array}{l}\text { Heterogeneity } \\
\left(I^{2}\right)\end{array}$ & $\begin{array}{l}\text { Number } \\
\text { of } \\
\text { interven- } \\
\text { tions }\end{array}$ & $\begin{array}{l}\text { Sample } \\
\text { Size }\end{array}$ & $\begin{array}{l}\text { GRADE } \\
\text { level }\end{array}$ \\
\hline \multicolumn{7}{|l|}{ Nausea incidence } \\
\hline $\begin{array}{l}\text { Overall nausea incidence: } \\
\text { any dose, any duration }\end{array}$ & $\begin{array}{l}\text { OR: } 1.0, \\
95 \% \mathrm{CI}: \\
0.74-1.28\end{array}$ & $\mathrm{P}=0.82^{\mathrm{b}}$ & $0 \%{ }^{\mathrm{c}}$ & 8 & 883 & moderate \\
\hline $\begin{array}{l}\text { Acute nausea incidence: } \\
\text { any dose, any duration }\end{array}$ & $\begin{array}{l}\text { OR: } 0.8 \text {, } \\
95 \% \mathrm{CI}: \\
0.47-1.42\end{array}$ & $\mathrm{P}=0.47^{\mathrm{b}}$ & $63 \%$ & 6 & 590 & very low \\
\hline $\begin{array}{l}\text { Delayed nausea incidence: } \\
\text { any dose, any duration }\end{array}$ & $\begin{array}{l}\text { OR: } 0.9 \text {, } \\
95 \% \mathrm{CI}: \\
0.65-1.30\end{array}$ & $\mathrm{P}=0.64^{\mathrm{b}}$ & $28 \%$ & 7 & 834 & moderate \\
\hline \multicolumn{7}{|l|}{ Nausea Severity } \\
\hline $\begin{array}{l}\text { Overall nausea severity: } \\
\text { any dose, any duration }\end{array}$ & $\begin{array}{l}\text { SMD: } 0.2 \text {, } \\
95 \% \text { CI: } \\
-0.02-0.38\end{array}$ & $\mathrm{P}=0.08$ & $0 \%^{\mathrm{c}}$ & 3 & 378 & low \\
\hline $\begin{array}{l}\text { Acute nausea severity: } \\
\text { any dose, any duration }\end{array}$ & $\begin{array}{l}\text { SMD: } 0 \text {, } \\
95 \% \text { CI: } \\
-0.17-0.23\end{array}$ & $\mathrm{P}=0.76^{\mathrm{b}}$ & $0 \%$ & 5 & 438 & low \\
\hline $\begin{array}{l}\text { Delayed nausea severity: } \\
\text { any dose, any duration }\end{array}$ & $\begin{array}{l}\text { SMD: } 0.4 \text {, } \\
95 \% \text { CI: } \\
-0.07-0.77\end{array}$ & $\mathrm{P}=0.10^{\mathrm{b}}$ & $75 \%$ & 4 & 378 & very low \\
\hline \multicolumn{7}{|l|}{ Vomiting Incidence } \\
\hline $\begin{array}{l}\text { Overall vomiting incidence: }^{a} \\
\text { any dose, any duration }\end{array}$ & $\begin{array}{l}\text { OR: } 0.8, \\
95 \% \mathrm{CI}: \\
0.44-1.36\end{array}$ & $\mathrm{P}=0.38$ & $66 \%$ & 9 & 825 & very low \\
\hline $\begin{array}{l}\text { Acute vomiting incidence: } \\
\text { any dose, any duration }\end{array}$ & $\begin{array}{l}\text { OR: } 0.7 \text {, } \\
95 \% \mathrm{CI}: \\
0.38-1.24\end{array}$ & $\mathrm{P}=0.22$ & $57 \%$ & 7 & 671 & Very low \\
\hline $\begin{array}{l}\text { Acute vomiting incidence: } \\
\leq 1 \text { g/day dose, }>3 \text { days duration }\end{array}$ & $\begin{array}{l}\text { OR: } 0.4 ; \\
95 \% \text { CI: } \\
0.17-0.81\end{array}$ & $\mathrm{P}=0.01$ & $20 \%$ & 3 & 301 & moderate \\
\hline
\end{tabular}




\begin{tabular}{|c|c|c|c|c|c|c|}
\hline $\begin{array}{l}\text { Delayed vomiting incidence: } \\
\text { any dose, any duration }\end{array}$ & $\begin{array}{l}\text { OR: } 0.8, \\
95 \% \text { CI: } \\
0.39-1.79\end{array}$ & $\mathrm{P}=0.63$ & $76 \%$ & 7 & 671 & very low \\
\hline \multicolumn{7}{|l|}{ Secondary Outcomes } \\
\hline $\begin{array}{l}\text { Quality of Life: } \\
\text { any dose, any duration }\end{array}$ & $\begin{array}{l}\text { SMD: } 0.5 \text {, } \\
95 \% \text { CI: } \\
-0.07-1.01\end{array}$ & $\mathrm{P}=0.09$ & $78 \%$ & 3 & 279 & low \\
\hline $\begin{array}{l}\text { Fatigue Incidence: } \\
\text { any dose, any duration }\end{array}$ & $\begin{array}{l}\text { OR: } 0.5 \text {, } \\
95 \% \mathrm{CI}: \\
0.13-1.61\end{array}$ & $\mathrm{P}=0.22$ & $60 \%$ & 4 & 375 & very low \\
\hline $\begin{array}{l}\text { Fatigue Incidence: } \\
\text { any dose, } \leq 3 \text {-days duration }\end{array}$ & $\begin{array}{l}\text { OR: } 0.2, \\
95 \% \mathrm{CI}: \\
0.03-0.87\end{array}$ & $P=0.03$ & $0 \%{ }^{\mathrm{c}}$ & 2 & 219 & Low \\
\hline \multicolumn{7}{|l|}{ Adverse Events } \\
\hline $\begin{array}{l}\text { Heartburn incidence: } \\
\text { any dose, any duration }\end{array}$ & $\begin{array}{l}\text { OR: } 1.9 \text {, } \\
95 \% \mathrm{CI}: \\
0.68-5.18\end{array}$ & $\mathrm{P}=0.22$ & $0 \%$ & 3 & 312 & low \\
\hline $\begin{array}{l}\text { Any gastrointestinal, flushing, rash- } \\
\text { related, or unspecified adverse } \\
\text { event: } \\
\text { any dose, any duration }\end{array}$ & $\begin{array}{l}\text { OR: } 2.0, \\
95 \% \mathrm{CI}: \\
1.39-2.99\end{array}$ & $P=0.0003$ & $0 \%$ & 5 & 1458 & moderate \\
\hline
\end{tabular}

a. Subgroup analysis based on the four a priori groups of varied duration and dosage were not significantly different from each other.

b. Sensitivity analysis did not identify a significant effect estimate

c. Improved with sensitivity analysis 


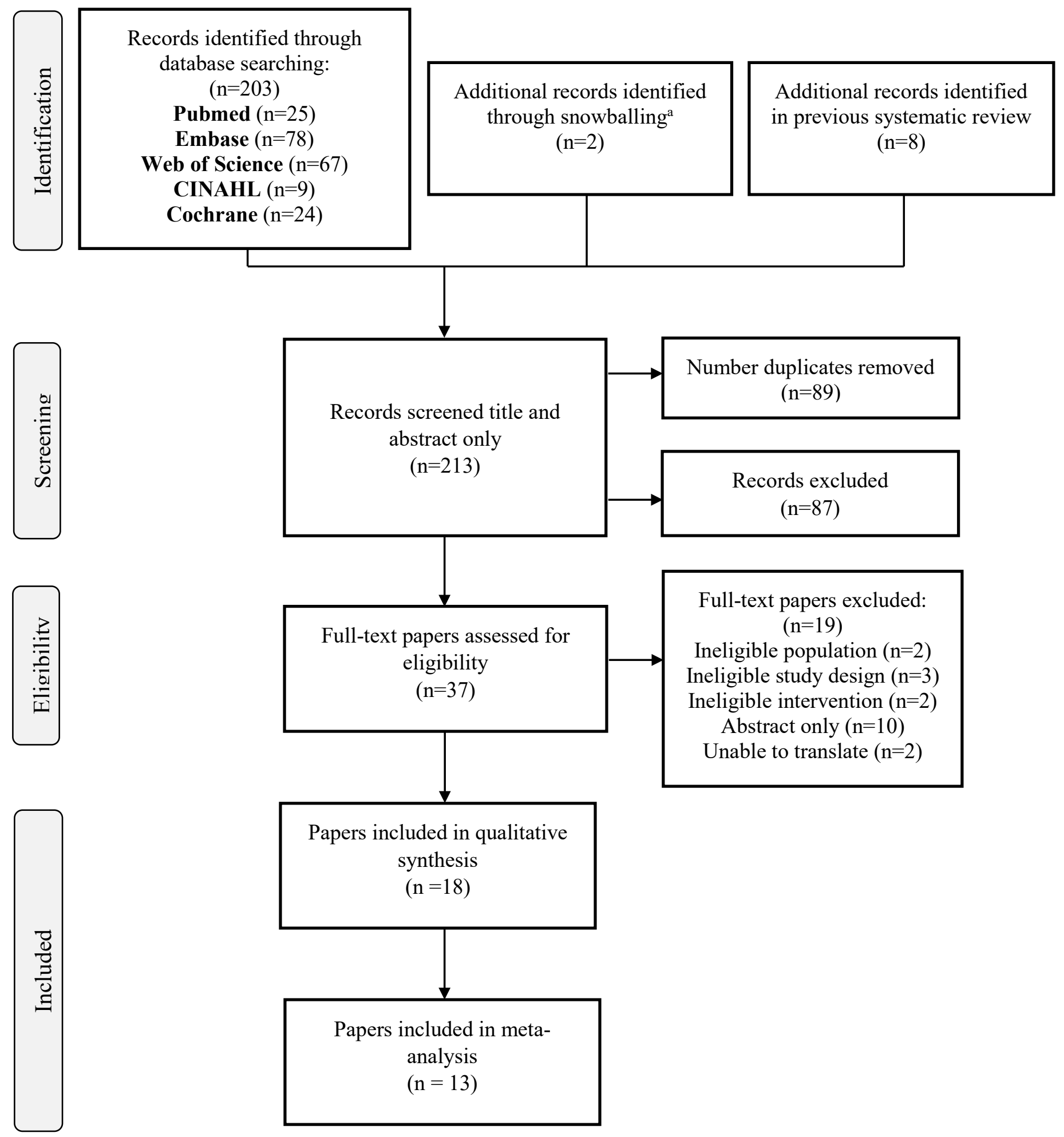

Figure 1. PRISMA flowchart of the search results and included papers in this systematic review with meta-analyses aiming to evaluate, in adult cancer patients receiving chemotherapy, the effects of ginger supplementation dose and duration on the incidence and severity of chemotherapy-induced nausea and vomiting and outcomes related to chemotherapy-induced nausea and vomiting (quality of life, fatigue). ${ }^{a}$ Reference lists of included studies and previous systematic and narrative reviews were examined to identify additional studies not found in the systematic search strategy. 


\begin{tabular}{|c|c|c|c|c|c|c|c|c|c|c|c|c|c|c|c|c|c|c|}
\hline $\begin{array}{l}\frac{1}{\bar{Z}} \\
\stackrel{\bar{x}}{0} \\
\stackrel{8}{0} \\
\end{array}$ & 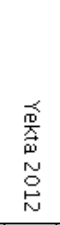 & 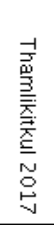 & $\begin{array}{l}\text { 訔 } \\
\text { 至 } \\
\stackrel{\circ}{\circ} \\
\stackrel{v}{v}\end{array}$ & 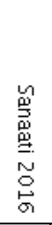 & 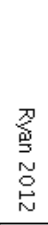 & 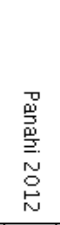 & 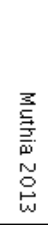 & 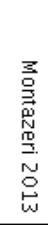 & 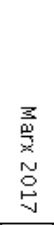 & 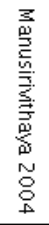 & $\begin{array}{l}\underset{\sim}{c} \\
\stackrel{\circ}{\circ}\end{array}$ & 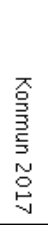 & 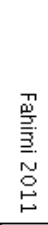 & 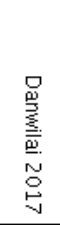 & 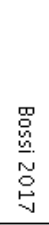 & 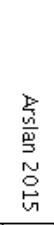 & 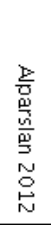 & \\
\hline$\odot$ & $\odot$ & $\odot$ & $\sim$ & $\odot$ & $\odot$ & (1) & $\sim$ & $\odot$ & + & $\odot$ & $\sim$ & $\odot$ & $\sim$ & $\odot$ & $\odot$ & $\curvearrowright$ & $\sim$ & Random sequence generation (selection bias) \\
\hline$\odot$ & $\sim$ & $\sim$ & $\sim$ & $\odot$ & $\curvearrowright$ & (1) & $\curvearrowright$ & $\sim$ & + & $\sim$ & $\sim$ & $\sim$ & $\sim$ & $\sim$ & $\sim$ & (1) & $\sim$ & Allocation concealment (selection bias) \\
\hline$\odot$ & + & + & $\odot$ & (1) & $\odot$ & (1) & (1) & + & + & $\odot$ & + & + & $\odot$ & + & + & (1) & (1) & Blinding of participants and personnel (performance bias) \\
\hline$\odot$ & $\odot$ & $\odot$ & $\odot$ & (1) & $\odot$ & (1) & (1) & $\odot$ & + & $\odot$ & $\odot$ & $\odot$ & $\odot$ & $\odot$ & $\odot$ & (1) & (1) & Blinding of outcome assessment (detection bias) \\
\hline$\odot$ & $\odot$ & $\odot$ & $\sim$ & (1) & $\odot$ & $\odot$ & $\sim$ & (1) & + & $\odot$ & $\odot$ & $\odot$ & $\sim$ & $\odot$ & $\sim$ & $\odot$ & $\sim$ & Incomplete outcome data (attrition bias) \\
\hline$\odot$ & + & $\odot$ & + & $\odot$ & 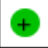 & + & + & $\sim$ & $\sim$ & + & + & + & + & + & $\odot$ & 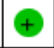 & + & Selective reporting (reporting bias) \\
\hline+ & + & + & $\odot$ & + & 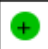 & + & $\odot$ & + & + & $\odot$ & + & $\odot$ & $\odot$ & $\odot$ & (1) & $\oplus$ & + & Other bias \\
\hline
\end{tabular}

Figure 2. Risk of bias summary: Judgements from the review authors about bias in each paper included in this systematic review with meta-analyses aiming to evaluate, in adult cancer patients receiving chemotherapy, the effects of ginger supplementation dose and duration on the incidence and severity of chemotherapy-induced nausea and vomiting and outcomes related to chemotherapy-induced nausea and vomiting (quality of life, fatigue). 
Ginger Comparator Odds Ratio Odds Ratio

Study or Subgroup Events Total Events Total Weight $\mathrm{M}-\mathrm{H}, \mathrm{Random}, 95 \% \mathrm{CI}$ 1.18.1 Short Duration Low Dose

$\begin{array}{llllll}21 & 36 & 27 & 36 & 14.8 \% & 0.47[0.17,1.27]\end{array}$

Zick 2009

Subtotal $(95 \% \mathrm{CI})$

Total events

$\begin{array}{lllll}53 & 14 & 57 & 17.1 \% & 1.72[0.75,3.91] \\ \mathbf{8 9} & & \mathbf{9 3} & \mathbf{3 1 . 9} \% & \mathbf{0 . 9 2}[0.26,3.31]\end{array}$

Heterogeneity. $\mathrm{Tau}^{2}=0.63 ; \mathrm{Chi}^{2}=3.86, \mathrm{df}=1(\mathrm{P}=0.05) ; \mathrm{I}^{2}=74 \%$

Test for overall effect: $Z=0.12(P=0.90)$

\subsubsection{Short Duration High Dose}

$\begin{array}{lllllll}\text { Zick 2009b } & 17 & 53 & 14 & 57 & 17.0 \% & 1.45[0.63,3.34] \\ \text { Subtotal }(\mathbf{9 5} \% \mathbf{C I}) & & \mathbf{5 3} & & \mathbf{5 7} & \mathbf{1 7 . 0 \%} & \mathbf{1 . 4 5}[\mathbf{0 . 6 3}, \mathbf{3 . 3 4}] \\ \text { Total events } & 17 & & 14 & & & \end{array}$

Total events

Heterogeneity. Not applicable

Test for overall effect: $Z=0.87(P=0.38$ )

1.18.3 Long Duration Low Dose

Konmun 2017

Li 2018

Yekta 2012

Subtotal $(95 \% \mathrm{CI})$

Total events

540

40
71

$\begin{array}{lll}18 & 41 & 13.4 \%\end{array}$

$11-69 \quad 14.1 \%$

14

Heterogeneity. $\mathrm{Tau}^{2}=0.10 ; \mathrm{Chi}^{2}=2.51, \mathrm{df}=2(\mathrm{P}=0.29) ; \mathrm{I}^{2}=20 \%$

Test for overall effect: $Z=2.50(P=0.01)$

1.18.4 Long Duration High Dose

Panahi 2012

Subtotal $(95 \% \mathrm{CI})$

$8 \quad 37$
$\quad 37$

$37 \quad 12$

$241 \quad 14.4 \%$

$0.67[0.24,1.87]$

Total events

$8 \quad 12$

Heterogeneity. Not applicable

Test for overall effect: $Z=0.77(P=0.44)$

Total $(95 \% \mathrm{Cl})$

330

Total events

79

100

$341 \quad 100.0 \%$

$0.69[0.38,1.24]$

$\mathrm{au}^{2}=0.35$

est for overall effect: $z=1.24(P=0.22)$

Test for subgroup differences: $C h \mathrm{I}^{2}=5.70, \mathrm{df}=3\left(\mathrm{P}=0.13 \mathrm{)}, \mathrm{I}^{2}=47.4 \%\right.$

$0.18[0.06,0.56]$

$0.49[0.17,1.40]$

$0.73[0.15,3.49]$

$0.37[0.17,0.81]$

Figure 3. The likelihood of acute vomiting was reduced in adults undergoing chemotherapy by $60 \%$ with ginger supplementation of $\leq 1 \mathrm{~g} /$ day for $>3$-days compared to control groups (OR: $0.4,95 \%$ CI: $0.17-0.81 ; \mathrm{P}=0.01 ; \mathrm{n}=3$ studies; $\mathrm{n}=3$ interventions; $\mathrm{n}=301$ participants; $\mathrm{I}^{2}=20 \%$; GRADE level: moderate). Short Duration $=\leq 3$-days. Long Duration $=>3$-days. Low Dose $=\leq 1 \mathrm{~g} /$ day. High dose $=>1 \mathrm{~g} /$ day. $\mathrm{OR}=$ Odds ratio. $\mathrm{CI}=$ Confidence Interval. 


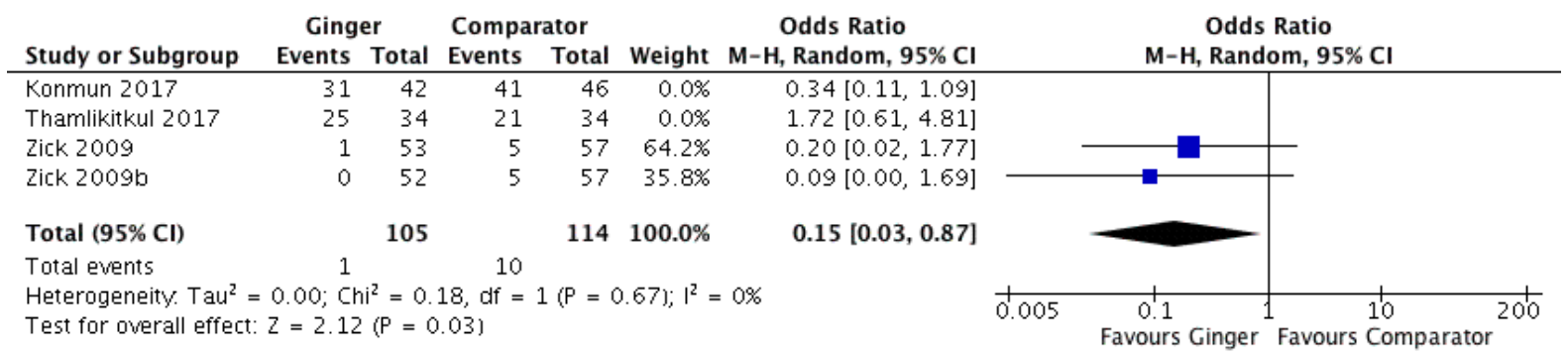

Figure 4. The likelihood of fatigue was reduced by $80 \%$ with ginger supplementation of any dose for $<3$-days duration compared to control groups (OR: $0.2,95 \%$ CI: $0.03-0.87$; $\mathrm{P}=0.03$; $n=1$ studies; $n=2$ interventions; $n=219$ participants; $I^{2}=0 \%$; GRADE level: low). Sensitivity analysis: studies which administered intervention for $>3$ days were excluded. $O R=O d d s$ ratio. $\mathrm{CI}=$ Confidence Interval. 


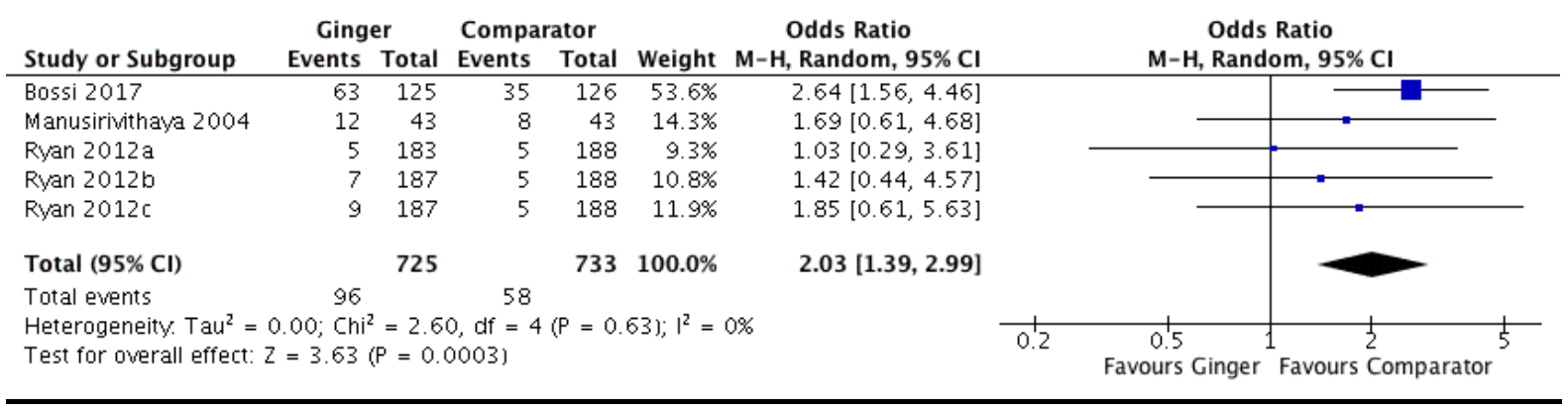

Figure 5. The likelihood of any gastrointestinal, flushing, rash-related or unspecified adverse event reasonably relatable to the intervention was increased in adults undergoing chemotherapy with ginger supplementation of any dose for any duration (OR: 2.0, 95\% CI: 1.39-2.99; $\mathrm{P}=0.0003 ; \mathrm{n}=3$ studies; $\mathrm{n}=5$ interventions; 1458 participants; $\mathrm{I}^{2}=0 \%$; GRADE level: moderate). $\mathrm{OR}=$ Odds ratio. $\mathrm{CI}=$ Confidence Interval. 


\section{Online Supplementary Material 1: Database Search Strategies}

\section{Pubmed Database:}

(ginger[MeSH] OR ginger*[tiab] OR zingiber officinale*[tiab] OR "officinales, zingiber"[tiab]) AND (neoplasms[MeSH] OR "chemotherapy, adjuvant" [MeSH] OR "consolidation chemotherapy" [MeSH] OR "induction chemotherapy" $[\mathrm{MeSH}]$ OR "photochemotherapy" $[\mathrm{MeSH}]$ OR "maintenance chemotherapy" [MeSH] OR "chemotherapy, cancer, regional perfusion" [MeSH] OR "antineoplastic combined chemotherapy protocols" [MeSH] OR "electrochemotherapy" [MeSH] OR neoplas*[tiab] OR tumor*[tiab] OR tumour*[tiab] OR malignanc*[tiab] OR cancer*[tiab] OR chemotherap*[tiab] OR "antineoplastic combined chemotherapy regimens" [tiab] OR antineoplastic chemotherapy protocol*[tiab] OR "chemotherapy protocol, antineoplastic" [tiab] OR "protocol, antineoplastic chemotherapy" [tiab] OR cancer chemotherapy protocol*[tiab] OR "protocol, cancer chemotherapy" [tiab] OR "adjuvant chemotherapy" [tiab] OR "consolidation chemotherapies" [tiab] OR "chemotherapy, consolidation" [tiab] OR "regional perfusion antineoplastic chemotherapy" [tiab] OR "isolation perfusion cancer chemotherapy" [tiab] OR "cancer chemotherapy, regional perfusion" [tiab] OR "perfusion cancer chemotherapy, regional" [tiab] OR "regional perfusion cancer chemotherapy" [tiab] OR electrochemotherapies OR "chemotherapy, induction" [tiab] OR "maintenance chemotherapies"[tiab] OR photochemotherapies[tiab] AND (nausea[MeSH] OR vomiting [MeSH] OR emetics[MeSH] OR Antiemetics[MeSH] OR emesis[tiab] OR emetogenic[tiab] OR emetogenicity[tiab] OR nausea[tiab] OR nauseous[tiab] OR vomit*[tiab] OR emetic*[tiab] OR regurgit*[tiab]) OR "chemotherapy induced nausea and vomiting" [tiab] OR "chemotherapy-induced nausea and vomiting" [tiab] OR CINV[tiab] AND (Randomized controlled trial[pt] OR controlled clinical trial[pt] OR clinical study[pt] OR clinical trial[pt] OR comparative study[pt] OR randomized[tiab] OR randomised[tiab] OR placebo[tiab] OR OR randomly[tiab] OR trial[tiab] OR groups[tiab] OR "Single blind"[tiab] OR “Double blind"[tiab] OR intervention[tiab])

\section{Web of Science Database:}

((ginger OR ginger* OR "zingiber officinale*" OR "officinales, zingiber") AND (neoplasms OR "chemotherapy, adjuvant" OR "consolidation chemotherapy" OR "induction chemotherapy" OR photochemotherapy OR "maintenance chemotherapy" OR "chemotherapy, cancer, regional perfusion" OR "antineoplastic combined chemotherapy protocols" OR electrochemotherapy OR neoplas* OR tumor* OR tumour* OR malignanc* OR cancer* OR chemotherap* OR "antineoplastic combined chemotherapy regimens" OR "antineoplastic chemotherapy protocol*" OR "chemotherapy protocol, antineoplastic" OR "protocol, antineoplastic chemotherapy" OR "cancer chemotherapy protocol*" OR "protocol, cancer chemotherapy" OR "adjuvant chemotherapy" OR "consolidation chemotherapies" OR "chemotherapy, consolidation" OR "regional perfusion antineoplastic chemotherapy" OR "isolation perfusion cancer chemotherapy" OR "cancer chemotherapy, regional perfusion" OR "perfusion cancer chemotherapy, regional" OR "regional perfusion cancer chemotherapy" OR electrochemotherapies OR "chemotherapy, induction" OR "maintenance chemotherapies" OR photochemotherapies OR "chemotherapy induced nausea and vomiting" OR "chemotherapy-induced nausea and vomiting" OR CINV) AND (nausea OR vomiting OR emetics OR Antiemetics OR emesis OR emetogenic OR emetogenicity OR nausea OR nauseous OR vomit* OR 
emetic* OR regurgit*) AND ("Randomized controlled trial" OR "controlled clinical trial" OR "clinical study" OR "clinical trial" OR "comparative study" OR randomized OR randomised OR placebo OR randomly OR trial OR groups OR "Single blind" OR "Double blind" OR intervention))

\section{Embase Database:}

('ginger'/exp OR ginger*:ti,ab OR 'zingiber officinale*':ti,ab OR 'officinales, zingiber':ti,ab) AND ('neoplasms'/exp OR 'chemotherapy, adjuvant'/exp OR 'consolidation chemotherapy'/exp OR 'induction chemotherapy'/exp OR 'photochemotherapy'/exp OR 'maintenance chemotherapy'/exp OR 'chemotherapy, cancer, regional perfusion'/exp OR 'antineoplastic combined chemotherapy protocols'/exp OR 'electrochemotherapy'/exp OR neoplas*:ti,ab OR tumor*:ti,ab OR tumour*:ti,ab OR malignanc*:ti,ab OR cancer*:ti,ab OR chemotherap*:ti,ab OR 'antineoplastic combined chemotherapy regimens':ti,ab OR 'antineoplastic chemotherapy protocol*':ti,ab OR 'chemotherapy protocol, antineoplastic':ti,ab OR 'protocol, antineoplastic chemotherapy':ti,ab OR 'cancer chemotherapy protocol*':ti,ab OR 'protocol, cancer chemotherapy':ti,ab OR 'adjuvant chemotherapy':ti,ab OR 'consolidation chemotherapies':ti,ab OR 'chemotherapy, consolidation':ti,ab $O R$ 'regional perfusion antineoplastic chemotherapy':ti,ab $O R$ 'isolation perfusion cancer chemotherapy':ti,ab OR 'cancer chemotherapy, regional perfusion':ti,ab OR 'perfusion cancer chemotherapy, regional':ti,ab OR 'regional perfusion cancer chemotherapy':ti,ab OR electrochemotherapies OR 'chemotherapy, induction':ti,ab OR 'maintenance chemotherapies':ti,ab OR photochemotherapies:ti,ab OR 'chemotherapy induced nausea and vomiting':ti,ab OR 'chemotherapy-induced nausea and vomiting':ti,ab OR cinv:ti,ab) AND ('nausea'/exp OR 'vomiting'/exp OR 'emetics'/exp OR 'antiemetics'/exp OR emesis:ti,ab OR emetogenic:ti,ab OR emetogenicity:ti,ab OR nausea:ti,ab OR nauseous:ti,ab OR vomit*:ti,ab OR emetic*:ti,ab OR regurgit*:ti,ab) AND ('randomized controlled trial':it OR 'controlled clinical trial':it OR 'clinical study':it OR 'clinical trial':it OR 'comparative study':it OR randomized:ti,ab OR randomised:ti,ab OR placebo:ti,ab OR randomly:ti,ab OR trial:ti,ab OR groups:ti,ab OR 'single blind':ti,ab OR 'double blind':ti,ab OR intervention:ti,ab)

\section{CINAHL Database:}

$\begin{array}{lllllllll}(((\mathrm{MH} \quad \text { "ginger+") } & \mathrm{OR} & \mathrm{TI} & \text { ginger* } & \mathrm{OR} & \mathrm{AB} & \text { ginger* } & \mathrm{OR} & \mathrm{TI}\end{array}$ "zingiber officinale*" $O R \quad A B$ "zingiber officinale*" $O R \quad T I$ "officinales, zingiber" $\mathrm{OR} \quad \mathrm{AB}$ "officinales, zingiber") AND ((MH "neoplasms+") OR (MH "chemotherapy, adjuvant+") $\mathrm{OR}$ ( $\mathrm{MH}$ "consolidation chemotherapy+") OR (MH "induction chemotherapy+") OR ( $\mathrm{MH}$ "photochemotherapy+") OR (MH "maintenance chemotherapy+") OR ( $\mathrm{MH}$ "chemotherapy, cancer, regional perfusion+") OR (MH "antineoplastic $\begin{array}{llll}\text { combined chemotherapy } & \text { protocolst") } & O R & \text { (MH }\end{array}$ "electrochemotherapy+") $O R \quad T I$ neoplas* $O R \quad A B$ neoplas* $O R$ TI tumor* $O R \quad A B$ tumor* OR TI tumour* OR AB tumour* OR TI malignanc* OR AB malignanc* OR TI cancer* OR AB cancer* $\mathrm{OR} \quad \mathrm{TI} \quad$ chemotherap* $\mathrm{OR} \quad \mathrm{AB} \quad$ chemotherap* $^{*} \mathrm{OR} \quad \mathrm{TI}$ "antineoplastic combined chemotherapy regimens" $O R \quad A B$ "antineoplastic combined chemotherapy regimens" OR TI "antineoplastic chemotherapy protocol*" OR AB "antineoplastic chemotherapy protocol*" OR TI "chemotherapy protocol, 
antineoplastic" $O R \quad A B \quad$ "chemotherapy protocol, antineoplastic" $O R \quad$ TI "protocol, antineoplastic chemotherapy" OR AB "protocol, antineoplastic chemotherapy" OR TI "cancer chemotherapy protocol*" OR AB "cancer chemotherapy protocol*" $O R \quad T I$ "protocol, cancer chemotherapy" $O R A B$ "protocol, cancer chemotherapy" OR TI "adjuvant chemotherapy" $O R$ AB "adjuvant chemotherapy" OR TI "consolidation chemotherapies" $O R \quad A B$ "consolidation chemotherapies" OR TI "chemotherapy, consolidation" $O R \quad A B$ "chemotherapy, consolidation" OR TI "regional perfusion antineoplastic chemotherapy" OR AB "regional perfusion antineoplastic chemotherapy" OR TI "isolation perfusion cancer chemotherapy" $O R \quad A B$ "isolation perfusion cancer chemotherapy" $O R \quad T I$ "cancer chemotherapy, regional perfusion" $O R \quad A B$ "cancer chemotherapy, regional perfusion" $O R \mathrm{TI}$ "perfusion cancer chemotherapy, regional" $O R A B$ "perfusion cancer chemotherapy, regional" $O R \quad \mathrm{TI}$ "regional perfusion cancer chemotherapy" $O R \quad A B$ "regional perfusion cancer chemotherapy" $O R$ electrochemotherapies $O R \quad T I$ "chemotherapy, induction" $O R A B$ "chemotherapy, induction" $O R \quad \mathrm{TI}$ "maintenance chemotherapies" $O R \quad A B$ "maintenance chemotherapies" $O R \quad T I$ photochemotherapies $O R \quad A B$ photochemotherapies $O R \quad T I$ "chemotherapy induced nausea and vomiting" $O R A B$ "chemotherapy induced nausea and vomiting" OR TI "chemotherapy-induced nausea and vomiting" $O R A B$ "chemotherapy-induced nausea and vomiting" OR TI CINV OR AB CINV AND ((MH "nausea+") OR (MH "vomiting+") OR (MH "emetics+") OR (MH "Antiemetics+") OR TI emesis OR AB emesis OR TI emetogenic $O R A B$ emetogenic $O R$ TI emetogenicity OR AB emetogenicity OR TI nausea OR AB nausea OR TI nauseous OR AB nauseous OR TI vomit* OR AB vomit* OR TI emetic* OR AB emetic* OR TI regurgit* OR AB regurgit*)))

\section{Cochrane Library Database:}

\#1 MeSH descriptor: [Ginger] explode all trees

\#2 MeSH descriptor: [Neoplasms] explode all trees

\#3 (ginger*:ti,ab or "zingiber officinale*":ti,ab or "officinales, zingiber":ti,ab)

\#4 \#1 or \#3

\#5 (neoplas*:ti,ab or tumor*:ti,ab or tumour*:ti,ab or malignanc*:ti,ab or cancer*:ti,ab or chemotherap*:ti,ab or "antineoplastic combined chemotherapy regimens":ti,ab or "antineoplastic chemotherapy protocol*":ti,ab or "chemotherapy protocol, antineoplastic":ti,ab or "protocol, antineoplastic chemotherapy":ti,ab or "cancer chemotherapy protocol*":ti,ab or "protocol, cancer chemotherapy":ti,ab or "adjuvant chemotherapy":ti,ab or "consolidation chemotherapies":ti,ab or "chemotherapy, consolidation":ti,ab or "regional perfusion antineoplastic chemotherapy":ti,ab or "isolation perfusion cancer chemotherapy":ti,ab or "cancer chemotherapy, regional perfusion":ti,ab or "perfusion cancer chemotherapy, regional":ti,ab or "regional perfusion cancer chemotherapy":ti,ab or electrochemotherapies or "chemotherapy, induction":ti,ab or "maintenance chemotherapies":ti,ab or photochemotherapies:ti,ab or "chemotherapy 
induced nausea and vomiting":ti,ab or "chemotherapy-induced nausea and vomiting":ti,ab or CINV:ti,ab)

\#6 MeSH descriptor: [Chemotherapy, Adjuvant] explode all trees

\#7 MeSH descriptor: [Consolidation Chemotherapy] explode all trees

\#8 MeSH descriptor: [Induction Chemotherapy] explode all trees

\#99 MeSH descriptor: [Photochemotherapy] explode all trees

\#10 MeSH descriptor: [Maintenance Chemotherapy] explode all trees

\#11 MeSH descriptor: [Chemotherapy, Cancer, Regional Perfusion] explode all trees

\#12 MeSH descriptor: [Antineoplastic Combined Chemotherapy Protocols] explode all trees

\#13 MeSH descriptor: [Electrochemotherapy] explode all trees

$\# 14 \# 2$ or \#5 or \#6 or \#7 or \#8 or \#9 or \#10 or \#11 or \#12 or \#13

\#15 MeSH descriptor: [Nausea] explode all trees

\#16 MeSH descriptor: [Vomiting] explode all trees

\#17 MeSH descriptor: [Emetics] explode all trees

\#18 MeSH descriptor: [Antiemetics] explode all trees

\#19 (emesis:ti,ab or emetogenic:ti,ab or emetogenicity:ti,ab or nausea:ti,ab or nauseous:ti,ab or vomit*:ti,ab or emetic*:ti,ab or regurgit*:ti,ab)

\#20 \#15 or \#16 or \#17 or \#18 or \#19

\#21 ("Randomized controlled trial":pt or "controlled clinical trial":pt or "clinical study":pt or "clinical trial":pt or "comparative study":pt or randomized:ti,ab or randomised:ti,ab or placebo:ti,ab or randomly:ti,ab or trial:ti,ab or groups:ti,ab or "Single blind":ti,ab or "Double blind":ti,ab or intervention:ti,ab)

$\# 22 \quad \# 4$ and \#14 and \#20 and \#21 
Online Supplementary Material 2: Cochrane Risk of Bias Assessment

Table 1. Cochrane Risk of Bias assessment with justifications for included studies located in the updated search ( $\mathrm{n}=13$ ), examining the effect of ginger supplementation on chemotherapy-induced nausea and vomiting incidence and related outcomes.

\begin{tabular}{|c|c|c|c|c|c|c|c|c|}
\hline & Study & $\begin{array}{l}\text { Random sequence } \\
\text { generation (selection } \\
\text { bias) }\end{array}$ & $\begin{array}{l}\text { Allocation } \\
\text { concealment } \\
\text { (selection bias) }\end{array}$ & $\begin{array}{l}\text { Blinding of } \\
\text { participants and } \\
\text { personnel } \\
\text { (performance bias) }\end{array}$ & $\begin{array}{l}\text { Blinding of outcome } \\
\text { assessment (detection } \\
\text { bias) }\end{array}$ & $\begin{array}{l}\text { Incomplete } \\
\text { outcome data } \\
\text { (attrition bias) }\end{array}$ & $\begin{array}{l}\text { Selective } \\
\text { reporting } \\
\text { (reporting bias) }\end{array}$ & Other bias \\
\hline Rating & & Risk of bias: Unclear & Risk of bias: Unclear & Risk of bias: High & Risk of bias: High & $\begin{array}{l}\text { Risk of bias: } \\
\text { Unclear }\end{array}$ & Low & Low \\
\hline Evidence & Alparslan 2012 & $\begin{array}{l}\text { No mention of } \\
\text { description of } \\
\text { randomisation or how } \\
\text { participants were } \\
\text { allocated to each group. } \\
\text { However, no difference } \\
\text { between baseline } \\
\text { characteristics ( }>0.05) \text {. }\end{array}$ & $\begin{array}{l}\text { No description of } \\
\text { how allocation was } \\
\text { conducted. }\end{array}$ & $\begin{array}{l}\text { No attempt at blinding } \\
\text { made (Intervention } \\
\text { group received ginger } \\
\text { tablet and no anti- } \\
\text { emetics, control group } \\
\text { received anti-emetics } \\
\text { and no ginger) } \\
\text { therefore outcomes } \\
\text { likely to be influenced } \\
\text { (more so nausea than } \\
\text { vomiting incidence) } \\
\text { and effect of } \\
\text { intervention may have } \\
\text { been overestimated. }\end{array}$ & $\begin{array}{l}\text { Vomiting incidence } \\
\text { objective, however, } \\
\text { nausea subjective and } \\
\text { assessment completed by } \\
\text { participants who were not } \\
\text { blinded to the } \\
\text { intervention or outcome, } \\
\text { therefore likely to effect } \\
\text { outcome and effect of } \\
\text { intervention may have } \\
\text { been overestimated. }\end{array}$ & $\begin{array}{l}\text { No raw data } \\
\text { provided (tables } \\
\text { missing from paper) } \\
\text { therefore no } \\
\text { indication of } \\
\text { attrition. }\end{array}$ & $\begin{array}{l}\text { No study protocol } \\
\text { identified, } \\
\text { however, all pre- } \\
\text { specified } \\
\text { expected } \\
\text { outcomes } \\
\text { included in } \\
\text { publication as } \\
\text { intended. }\end{array}$ & $\begin{array}{l}\text { The study appears } \\
\text { to be free of other } \\
\text { sources of bias. }\end{array}$ \\
\hline Rating & & Risk of bias: High & Risk of bias: High & Risk of bias: High & Risk of bias: High & $\begin{array}{l}\text { Risk of bias: } \\
\text { Unclear }\end{array}$ & $\begin{array}{l}\text { Risk of bias: } \\
\text { Low }\end{array}$ & Risk of bias: Low \\
\hline Evidence & Arslan 2015 & $\begin{array}{l}\text { "No significant } \\
\text { differences were seen } \\
\text { between the intervention } \\
\text { and control group } \\
\text { (p>0.05)" however, the } \\
\text { raw data was not } \\
\text { reported and the patients } \\
\text { were "randomized } \\
\text { sequentially to the two } \\
\text { groups (control and } \\
\text { intervention); that is, the } \\
\text { first patient was } \\
\text { assigned to the control } \\
\text { group and the next one } \\
\text { to the intervention } \\
\text { group." I.e. alternate } \\
\text { allocation / quasi- } \\
\text { randomisation used } \\
\end{array}$ & $\begin{array}{l}\text { The patients were } \\
\text { randomised } \\
\text { sequentially to the } \\
\text { two groups (control } \\
\text { and intervention); } \\
\text { that is, the first } \\
\text { patient was assigned } \\
\text { to the control group } \\
\text { and the next one to } \\
\text { the intervention } \\
\text { group, therefore } \\
\text { allocation } \\
\text { concealment } \\
\text { inadequate. }\end{array}$ & $\begin{array}{l}\text { No attempt at blinding } \\
\text { made (Intervention } \\
\text { group received ginger } \\
\text { powder, control group } \\
\text { received nothing) } \\
\text { therefore outcomes } \\
\text { likely to be influenced } \\
\text { (more so nausea than } \\
\text { vomiting incidence) } \\
\text { and effect of } \\
\text { intervention may have } \\
\text { been overestimated. }\end{array}$ & $\begin{array}{l}\text { "The patient diary was } \\
\text { given to the patients in } \\
\text { the intervention and } \\
\text { control groups, and they } \\
\text { were asked to complete } \\
\text { the diary four times a day } \\
\text { at home". Vomitting } \\
\text { incidence objective, } \\
\text { however, nausea } \\
\text { subjective and } \\
\text { assessment completed by } \\
\text { participants who were not } \\
\text { blinded to the } \\
\text { intervention, therefore } \\
\text { likely to effect outcome } \\
\text { and effect of intervention } \\
\text { may have been } \\
\text { overestimated. }\end{array}$ & $\begin{array}{l}\text { No mention of } \\
\text { attrition or sample } \\
\text { size per group. }\end{array}$ & $\begin{array}{l}\text { No study protocol } \\
\text { identified, } \\
\text { however, all pre- } \\
\text { specified } \\
\text { expected } \\
\text { outcomes } \\
\text { included in } \\
\text { publication as } \\
\text { intended. }\end{array}$ & $\begin{array}{l}\text { The study appears } \\
\text { to be free of other } \\
\text { sources of bias. }\end{array}$ \\
\hline
\end{tabular}




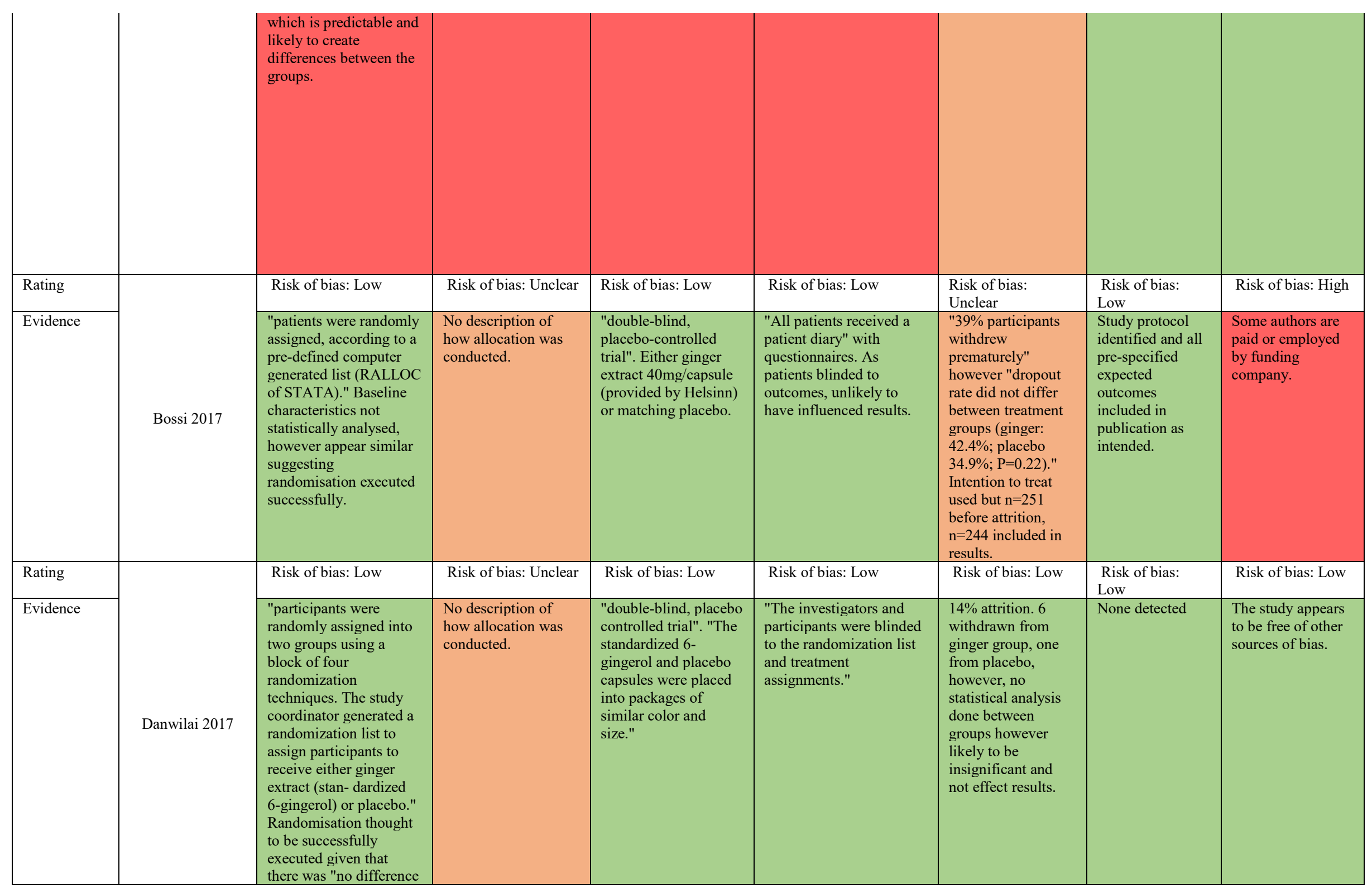




\begin{tabular}{|c|c|c|c|c|c|c|c|c|}
\hline & & $\begin{array}{l}\text { between baseline } \\
\text { characteristics } \\
(\mathrm{P}>0.05) . "\end{array}$ & & & & & & \\
\hline Rating & & Risk of bias: Low & Risk of bias: Unclear & Risk of bias: Low & Risk of bias: Low & Risk of bias: Low & $\begin{array}{l}\text { Risk of bias: } \\
\text { Low }\end{array}$ & Risk of bias: Low \\
\hline Evidence & Konmun 2017 & $\begin{array}{l}\text { Particpants were } \\
\text { "randomly assigned... } \\
\text { utilizing a block four } \\
\text { method for } \\
\text { randomization." } \\
\text { Randomisation thought } \\
\text { to be successfully } \\
\text { executed given that } \\
\text { there were no } \\
\text { differences between } \\
\text { baseline characteristics } \\
\text { reported. }\end{array}$ & $\begin{array}{l}\text { No description of } \\
\text { how allocation was } \\
\text { conducted. }\end{array}$ & $\begin{array}{l}\text { "double-blind, } \\
\text { placebo-controlled } \\
\text { trial" "placebo } \\
\text { capsules ... match the } \\
\text { weight of the 6- } \\
\text { gingerol capsules" }\end{array}$ & $\begin{array}{l}\text { "patients were required to } \\
\text { complete a daily diary ... } \\
\text { The diary included } \\
\text { number of vomiting } \\
\text { episodes, nausea score, } \\
\text { appetite score, quality of } \\
\text { life, use of rescue anti- } \\
\text { emetic, and } \\
\text { hospitalization." As } \\
\text { patients blinded to } \\
\text { outcomes well, unlikely } \\
\text { to have influenced results. }\end{array}$ & $\begin{array}{l}14 \% \text { attrition. } \\
\text { Withdrawal } \\
\text { numbers and } \\
\text { reasons similar } \\
\text { between groups. } \\
\text { Atrrition well } \\
\text { documented. }\end{array}$ & $\begin{array}{l}\text { No study protocol } \\
\text { identified, } \\
\text { however, all pre- } \\
\text { specified } \\
\text { expected } \\
\text { outcomes } \\
\text { included in } \\
\text { publication as } \\
\text { intended. }\end{array}$ & $\begin{array}{l}\text { The study appears } \\
\text { to be free of other } \\
\text { sources of bias. }\end{array}$ \\
\hline Rating & & Risk of bias: Unclear & Risk of bias: Unclear & Risk of bias: Low & Risk of bias: Low & Risk of bias: Low & Risk of bias: Low & Risk of bias: Low \\
\hline Evidence & Li 2018 & $\begin{array}{l}\text { No mention of } \\
\text { description of } \\
\text { randomisation or how } \\
\text { participants were } \\
\text { allocated to each group. } \\
\text { However, no difference } \\
\text { between baseline } \\
\text { characteristics ( } P>0.05 \text { ). }\end{array}$ & $\begin{array}{l}\text { No description of } \\
\text { how allocation was } \\
\text { conducted. }\end{array}$ & $\begin{array}{l}\text { "double-blind, } \\
\text { placebo-controlled } \\
\text { clini- cal trial" "The } \\
\text { placebo capsules were } \\
\text { physically identical to } \\
\text { the ginger capsules" }\end{array}$ & $\begin{array}{l}\text { Both participants and } \\
\text { researchers blinded to } \\
\text { outcomes. }\end{array}$ & $\begin{array}{l}4 \% \text { attrition. } \\
\text { Reasons for this and } \\
\text { numbers per group } \\
\text { given. No intention } \\
\text { to treat used } \\
\text { however, } 6 \text { drop } \\
\text { outs unlikely to } \\
\text { influence results. }\end{array}$ & $\begin{array}{l}\text { No study protocol } \\
\text { identified, } \\
\text { however, all pre- } \\
\text { specified } \\
\text { expected } \\
\text { outcomes } \\
\text { included in } \\
\text { publication as } \\
\text { intended. }\end{array}$ & $\begin{array}{l}\text { The study appears } \\
\text { to be free of other } \\
\text { sources of bias. }\end{array}$ \\
\hline Rating & Marx 2017 & Risk of bias: Low & Risk of bias: Low & Risk of bias: Low & Risk of bias: Low & Risk of bias: Low & $\begin{array}{l}\text { Risk of bias: } \\
\text { Unclear }\end{array}$ & Risk of bias: Low \\
\hline
\end{tabular}




\begin{tabular}{|c|c|c|c|c|c|c|c|c|}
\hline Evidence & & $\begin{array}{l}\text { "randomly allocated... } \\
\text { by an independent } \\
\text { company using a } \\
\text { computer-generated } \\
\text { sequence." Baseline } \\
\text { characteristics not } \\
\text { statistically analysed, } \\
\text { however appear similar } \\
\text { suggesting } \\
\text { randomisation executed } \\
\text { successfully. }\end{array}$ & $\begin{array}{l}\text { "Eligible patients } \\
\text { were randomly } \\
\text { allocated to ginger or } \\
\text { placebo capsules by } \\
\text { an independent } \\
\text { company using a } \\
\text { computer-generated } \\
\text { sequence." }\end{array}$ & $\begin{array}{l}\text { "All staff members } \\
\text { involved in } \\
\text { recruitment and } \\
\text { outcome assessment } \\
\text { were blinded to the } \\
\text { results of } \\
\text { randomization." } \\
\text { Placebo capsules same } \\
\text { appearance and } \\
\text { weight. Double- } \\
\text { enapsulated. }\end{array}$ & $\begin{array}{l}\text { Participants self- } \\
\text { completed questionnaires } \\
\text { and blinded to outcomes. }\end{array}$ & $\begin{array}{l}\text { Intention to treat } \\
\text { used ( } 34 / 51 \\
\text { completed all } \\
\text { cycles, however, } 51 \\
\text { included in } \\
\text { analysis). Attrition } \\
\text { well documented. }\end{array}$ & $\begin{array}{l}\text { Results reported } \\
\text { differ from those } \\
\text { pre-specified in } \\
\text { study protocol, } \\
\text { however, } \\
\text { explanations } \\
\text { provided in } \\
\text { publication. }\end{array}$ & $\begin{array}{l}\text { The study appears } \\
\text { to be free of other } \\
\text { sources of bias. }\end{array}$ \\
\hline Rating & & Risk of bias: Low & Risk of bias: Unclear & Risk of bias: Low & Risk of bias: Low & Risk of bias: High & $\begin{array}{l}\text { Risk of bias: } \\
\text { Unclear }\end{array}$ & Risk of bias: Low \\
\hline Evidence & Montazeri 2013 & $\begin{array}{l}\text { "This study is a } \\
\text { randomized, } \\
\text { prospective, cross-over } \\
\text { double - blinded clinical } \\
\text { trial," "This study was } \\
\text { doing on the basis of the } \\
\text { block randomization } \\
\text { with the four block } \\
\text { method." }\end{array}$ & $\begin{array}{l}\text { No description of } \\
\text { how allocation was } \\
\text { conducted. }\end{array}$ & $\begin{array}{l}\text { "Double-blind, } \\
\text { placebo-controlled } \\
\text { trial" "The shape, } \\
\text { colour and fragrance } \\
\text { of this powder were } \\
\text { similar to ginger and } \\
\text { both of them were } \\
\text { provided by the same } \\
\text { company." }\end{array}$ & $\begin{array}{l}\text { Both participants and } \\
\text { researchers blinded to } \\
\text { outcomes. }\end{array}$ & $\begin{array}{l}32 \% \text { attrition. } 13 / 44 \\
\text { unable to complete } \\
\text { second cycle of } \\
\text { study. Reasons for } \\
\text { this given but } \\
\text { dropouts per group } \\
\text { not given. Unclear } \\
\text { whether intention to } \\
\text { treat used. }\end{array}$ & $\begin{array}{l}\text { Unclear whether } \\
\text { outcome data } \\
\text { missing from } \\
\text { analysis and } \\
\text { whether this } \\
\text { could have effect } \\
\text { on results. }\end{array}$ & $\begin{array}{l}\text { The study appears } \\
\text { to be free of other } \\
\text { sources of bias. }\end{array}$ \\
\hline Rating & & Risk of bias: Unclear & Risk of bias: Unclear & Risk of bias: High & Risk of bias: High & $\begin{array}{l}\text { Risk of bias: } \\
\text { Unclear }\end{array}$ & $\begin{array}{l}\text { Risk of bias: } \\
\text { Low }\end{array}$ & Risk of bias: Low \\
\hline Evidence & Muthia 2013 & $\begin{array}{l}\text { No mention or } \\
\text { description of } \\
\text { randomisation or how } \\
\text { participants were } \\
\text { allocated to each group. } \\
\text { No statistical analysis of } \\
\text { baseline characteristics } \\
\text { to determine whether } \\
\text { sequence generation free } \\
\text { from bias. }\end{array}$ & $\begin{array}{l}\text { No description of } \\
\text { how allocation was } \\
\text { conducted. }\end{array}$ & $\begin{array}{l}\text { No attempt at blinding } \\
\text { made (Intervention } \\
\text { group received ginger } \\
\text { drink, control group } \\
\text { did not) therefore } \\
\text { outcome of vomiting } \\
\text { and mainly nausea } \\
\text { likely to be influenced } \\
\text { and effect of } \\
\text { intervention may have } \\
\text { been overestimated. } \\
\end{array}$ & $\begin{array}{l}\text { Rhodes Index for Nausea, } \\
\text { Vomiting and Retching } \\
\text { subjective and seeing as } \\
\text { participants and } \\
\text { researchers not blinded to } \\
\text { treatment and outcomes, } \\
\text { likely to influence results } \\
\text { and effect of intervention } \\
\text { may have been } \\
\text { overestimated. }\end{array}$ & $\begin{array}{l}\text { No mention of } \\
\text { attrition or sample } \\
\text { size per group. }\end{array}$ & $\begin{array}{l}\text { No study protocol } \\
\text { identified, } \\
\text { however, all pre- } \\
\text { specified } \\
\text { expected } \\
\text { outcomes } \\
\text { included in } \\
\text { publication as } \\
\text { intended. }\end{array}$ & $\begin{array}{l}\text { The study appears } \\
\text { to be free of other } \\
\text { sources of bias. }\end{array}$ \\
\hline Rating & Sanaati 2016 & Risk of bias: Low & Risk of bias: Low & Risk of bias: High & Risk of bias: High & Risk of bias: High & $\begin{array}{l}\text { Risk of bias: } \\
\text { Low }\end{array}$ & Risk of bias: Low \\
\hline
\end{tabular}




\begin{tabular}{|c|c|c|c|c|c|c|c|c|}
\hline Evidence & & $\begin{array}{l}\text { "randomly } \\
\text { allocated ....using the } 20 \\
\text { block random tables." } \\
\text { Randomisation thought } \\
\text { to be successfully } \\
\text { executed given that } \\
\text { there was no difference } \\
\text { between baseline } \\
\text { characteristics reported } \\
(\mathrm{P}>0.05) \text {. }\end{array}$ & $\begin{array}{l}\text { Coding and blinding } \\
\text { of the two groups } \\
\text { were performed } \\
\text { privately by the } \\
\text { pharmacologist } \\
\text { consultant. }\end{array}$ & $\begin{array}{l}\text { Control group given } \\
\text { no intervention, } \\
\text { treatment groups } \\
\text { aware receiving } \\
\text { intervention and only } \\
\text { blinded to whether } \\
\text { receiving ginger or } \\
\text { chamomile capsules. } \\
\text { Outcome likely to be } \\
\text { influenced and effect } \\
\text { of intervention may } \\
\text { have been } \\
\text { overestimated. }\end{array}$ & $\begin{array}{l}\text { "A self-made, two-part } \\
\text { self- reporting instrument } \\
\text { was used to measure the } \\
\text { frequency and severity of } \\
\text { nausea and vomiting". } \\
\text { Frequency of vomitting } \\
\text { objective, however, } \\
\text { nausea frequency/severity } \\
\text { and vomitting severity } \\
\text { subjective outcomes } \\
\text { likely to be influenced by } \\
\text { the fact that participants } \\
\text { were not blinded to } \\
\text { receiving treatment/not } \\
\text { and effect of intervention } \\
\text { may have been } \\
\text { overestimated. }\end{array}$ & $\begin{array}{l}30 \% \text { attrition. } 13 \\
\text { out of } 43 \\
\text { interrupted their } \\
\text { participation } \\
\text { (excluding } \\
\text { chamomile group). } \\
\text { Reasons given, } \\
\text { however, no } \\
\text { differentiation } \\
\text { between groups and } \\
\text { number of drop outs } \\
\text { per group not given. } \\
\text { No intention to treat } \\
\text { used. }\end{array}$ & $\begin{array}{l}\text { Study Protocol } \\
\text { pre-registered and } \\
\text { all pre-specified } \\
\text { expected } \\
\text { outcomes } \\
\text { included in } \\
\text { publication as } \\
\text { intended. }\end{array}$ & $\begin{array}{l}\text { The study appears } \\
\text { to be free of other } \\
\text { sources of bias. }\end{array}$ \\
\hline Rating & & Risk of bias: Unclear & Risk of bias: Unclear & Risk of bias: Low & Risk of bias: Low & $\begin{array}{l}\text { Risk of bias: } \\
\text { Unclear }\end{array}$ & $\begin{array}{l}\text { Risk of bias: } \\
\text { Low }\end{array}$ & Risk of bias: Low \\
\hline Evidence & Shokri 2017 & $\begin{array}{l}\text { "The patients were } \\
\text { divided into } 2 \text { random } \\
\text { groups. They were then } \\
\text { divided into } 2 \\
\text { homogeneous groups } \\
\text { (using Randlist) based } \\
\text { on their satisfaction to } \\
\text { receive ginger in terms } \\
\text { of the history of lack of } \\
\text { neoplastic diseases in } \\
\text { women, history of lack } \\
\text { of chemotherapy and } \\
\text { stage of cancer." } \\
\text { Unclear whether those } \\
\text { that were happy to } \\
\text { receive and comply with } \\
\text { ginger intervention more } \\
\text { likely to be allocated } \\
\text { that intervention. } \\
\text { However, no statistically } \\
\text { signficant differences } \\
\text { between other baseline } \\
\text { characteristcs. }\end{array}$ & $\begin{array}{l}\text { No description of } \\
\text { how allocation was } \\
\text { conducted. }\end{array}$ & $\begin{array}{l}\text { "Examiners and the } \\
\text { patients were unaware } \\
\text { of the coding and the } \\
\text { real grouping was only } \\
\text { specified after } \\
\text { statistical analysis." } \\
\text { Placebo used. }\end{array}$ & $\begin{array}{l}\text { No indication how nausea } \\
\text { and vomitting outcome } \\
\text { assessed. As participants } \\
\text { and researchers blinded to } \\
\text { intervention, unlikely to } \\
\text { effect outcomes. }\end{array}$ & $\begin{array}{l}\text { No mention of } \\
\text { attrition or sample } \\
\text { size per group. }\end{array}$ & $\begin{array}{l}\text { Study Protocol } \\
\text { pre-registered and } \\
\text { all pre-specified } \\
\text { expected } \\
\text { outcomes } \\
\text { included in } \\
\text { publication as } \\
\text { intended. }\end{array}$ & $\begin{array}{l}\text { The study appears } \\
\text { to be free of other } \\
\text { sources of bias. }\end{array}$ \\
\hline Rating & Thamlikitkul 2017 & Risk of bias: Low & Risk of bias: Unclear & Risk of bias: Low & Risk of bias: Low & Risk of bias: Low & $\begin{array}{l}\text { Risk of bias: } \\
\text { Low }\end{array}$ & Risk of bias: Low \\
\hline
\end{tabular}




\begin{tabular}{|c|c|c|c|c|c|c|c|c|}
\hline Evidence & & $\begin{array}{l}\text { "subjects were randomly } \\
\text { assigned in a } 1: 1 \text { ratio } \\
\text { from the stratified } \\
\text { randomization table." } \\
\text { Baseline characteristics } \\
\text { not reported per group } \\
\text { due to cross over design. }\end{array}$ & $\begin{array}{l}\text { No description of } \\
\text { how allocation was } \\
\text { conducted. }\end{array}$ & $\begin{array}{l}\text { "The placebo and } \\
\text { ginger capsules and } \\
\text { their packaging were } \\
\text { physically identical. } \\
\text { The investigators and } \\
\text { subjects were blinded } \\
\text { to the randomization } \\
\text { list and treatment } \\
\text { assignments." }\end{array}$ & $\begin{array}{l}\text { Participants filled out } \\
\text { questionnaires and were } \\
\text { blinded to outcome } \\
\text { measures and } \\
\text { intervention, therefore } \\
\text { unlikely to effect } \\
\text { outcomes. }\end{array}$ & $\begin{array}{l}\text { No attrition, all } \\
\text { participants } \\
\text { completed the } \\
\text { study. }\end{array}$ & $\begin{array}{l}\text { Study Protocol } \\
\text { pre-registered and } \\
\text { all pre-specified } \\
\text { expected } \\
\text { outcomes } \\
\text { included in } \\
\text { publication as } \\
\text { intended. }\end{array}$ & $\begin{array}{l}\text { The study appears } \\
\text { to be free of other } \\
\text { sources of bias. }\end{array}$ \\
\hline Rating & & Risk of bias: Low & Risk of bias: Unclear & Risk of bias: Low & Risk of bias: Low & Risk of bias: Low & $\begin{array}{l}\text { Risk of bias: } \\
\text { Low }\end{array}$ & Risk of bias: Low \\
\hline Evidence & Yekta 2012 & $\begin{array}{l}\text { "The patients were } \\
\text { randomly } \\
\text { allocated...using the } 20 \\
\text { block random tables." } \\
\text { No statistically } \\
\text { signficant differences } \\
\text { between baseline } \\
\text { characteristcs suggesting } \\
\text { randomisation executed } \\
\text { successfully. }\end{array}$ & $\begin{array}{l}\text { No description of } \\
\text { how allocation was } \\
\text { conducted. }\end{array}$ & $\begin{array}{l}\text { "placebo capsules ... } \\
\text { exactly the same size, } \\
\text { shape, color, taste, and } \\
\text { dosage as Zintoma } \\
\text { capsules." "Coding } \\
\text { and blinding of the } 2 \\
\text { groups were } \\
\text { performed privately } \\
\text { by the pharmacologist } \\
\text { consultant, and all of } \\
\text { the samples, data } \\
\text { analyzers, and all } \\
\text { participants too, were } \\
\text { unaware of the real } \\
\text { content of the capsule" }\end{array}$ & $\begin{array}{l}\text { Participants used self- } \\
\text { reporting instruments to } \\
\text { document outcomes and } \\
\text { seeing as participants } \\
\text { were blinded to } \\
\text { intervention, unlikely to } \\
\text { have effected results. } \\
\text { Outcome of vomiting } \\
\text { incidence objective rather } \\
\text { than subjective. }\end{array}$ & $\begin{array}{l}18 \% \text { attrition } \\
\text { relatively low, } \\
\text { reasons given for } \\
\text { this along with } \\
\text { numbers per group. } \\
\text { No intention to treat } \\
\text { used however, } 18 \\
\text { drop outs unlikely } \\
\text { to influence results. }\end{array}$ & $\begin{array}{l}\text { Study Protocol } \\
\text { pre-registered and } \\
\text { all pre-specified } \\
\text { expected } \\
\text { outcomes } \\
\text { included in } \\
\text { publication as } \\
\text { intended. }\end{array}$ & $\begin{array}{l}\text { The study appears } \\
\text { to be free of other } \\
\text { sources of bias. }\end{array}$ \\
\hline
\end{tabular}


Table 2. Cochrane Risk of Bias assessment with justifications for included studies located in the researchers' previous systematic review (n=5), examining the effect of ginger supplementation on chemotherapy-induced nausea and vomiting incidence and related outcomes.

\begin{tabular}{|c|c|c|c|c|c|c|c|c|}
\hline Rating & & Risk of bias: Unclear & Risk of bias: Unclear & Risk of bias: Low & Risk of bias: Low & $\begin{array}{l}\text { Risk of bias: } \\
\text { Unclear }\end{array}$ & Risk of bias: Low & $\begin{array}{l}\text { Risk of bias: } \\
\text { Low }\end{array}$ \\
\hline Evidence & Fahimi 2011 & $\begin{array}{l}\text { Participants randomised, } \\
\text { however, specific } \\
\text { information on how this } \\
\text { was carried out not given. } \\
\text { Baseline characteristics } \\
\text { not reported per group } \\
\text { due to cross over design. }\end{array}$ & $\begin{array}{l}\text { "The monitoring } \\
\text { investigator dispensed } \\
\text { either active drug or } \\
\text { placebo according to } \\
\text { the randomization } \\
\text { table." No information } \\
\text { given as to whether or } \\
\text { not this was concealed. }\end{array}$ & $\begin{array}{l}\text { Placebo capsules were } \\
\text { "identical". Participants } \\
\text { were blinded to the } \\
\text { intervention received also. }\end{array}$ & $\begin{array}{l}\text { Questionnaires given to } \\
\text { particpant to complete, } \\
\text { and seeing as patients } \\
\text { blinded to intervention, } \\
\text { unlikely to have effected } \\
\text { results. }\end{array}$ & $\begin{array}{l}28 \% \text { attrrition. } \\
36 / 50 \text { completed the } \\
\text { study. Reasons } \\
\text { given, however, did } \\
\text { not specify which } \\
\text { intervention they } \\
\text { were undergoing } \\
\text { when they } \\
\text { discontinued } \\
\text { therefore unsure } \\
\text { whether relatable to } \\
\text { the ginger } \\
\text { intervention. } \\
\text { Unclear whether } \\
\text { intention to treat } \\
\text { used. }\end{array}$ & $\begin{array}{l}\text { No study protocol } \\
\text { identified, } \\
\text { however, all pre- } \\
\text { specified expected } \\
\text { outcomes } \\
\text { included in } \\
\text { publication as } \\
\text { intended. }\end{array}$ & $\begin{array}{l}\text { The study appears } \\
\text { to be free of other } \\
\text { sources of bias. }\end{array}$ \\
\hline Rating & & Risk of bias: Low & Risk of bias: Unclear & Risk of bias: Low & Risk of bias: Low & Risk of bias: Low & Risk of bias: Low & $\begin{array}{l}\text { Risk of bias: } \\
\text { Low }\end{array}$ \\
\hline Evidence & $\begin{array}{c}\text { Manusirivithaya } \\
2004\end{array}$ & $\begin{array}{l}\text { Participants were } \\
\text { "randomly assigned by } \\
\text { block of four." Baseline } \\
\text { characteristics not } \\
\text { reported per group due to } \\
\text { cross over design. }\end{array}$ & $\begin{array}{l}\text { No description of how } \\
\text { allocation was } \\
\text { conducted. }\end{array}$ & $\begin{array}{l}\text { Participants and research } \\
\text { team blinded to } \\
\text { intervention. "All of these } \\
\text { capsules were identical in } \\
\text { appearance, contour, size, } \\
\text { and color." }\end{array}$ & $\begin{array}{l}\text { Scale of nausea severity } \\
\text { self-reported. Both } \\
\text { participants and } \\
\text { researchers blinded to } \\
\text { outcomes. }\end{array}$ & $\begin{array}{l}10 \% \text { attrition. } \\
\text { Reasons not given, } \\
\text { however numbers } \\
\text { per regimen given } \\
\text { and same for each } \\
\text { group. Intention to } \\
\text { treat not used ( } 48 \\
\text { recruited, } 43 \text { only } \\
\text { included in data } \\
\text { analysis), however, } \\
\text { not likely to effect } \\
\text { results. }\end{array}$ & $\begin{array}{l}\text { No study protocol } \\
\text { identified, } \\
\text { however, all pre- } \\
\text { specified expected } \\
\text { outcomes } \\
\text { included in } \\
\text { publication as } \\
\text { intended. }\end{array}$ & $\begin{array}{l}\text { The study appears } \\
\text { to be free of other } \\
\text { sources of bias. }\end{array}$ \\
\hline Rating & Panahi 2012 & Risk of bias: High & Risk of bias: High & Risk of bias: High & Risk of bias: High & Risk of bias: Low & Risk of bias: Low & $\begin{array}{l}\text { Risk of bias: } \\
\text { Low }\end{array}$ \\
\hline
\end{tabular}




\begin{tabular}{|c|c|c|c|c|c|c|c|c|}
\hline Evidence & & $\begin{array}{l}\text { "The participants of this } \\
\text { open-label trial were } \\
\text { individually and } \\
\text { alternatively allocated to } \\
\text { ginger or control group } \\
\text { with the first allocation } \\
\text { being chosen randomly. } \\
\text { At the beginning of study } \\
\text { and before patient } \\
\text { recruitment, blank } \\
\text { questionnaires were } \\
\text { numbered and } \\
\text { alternatively coded as } \\
\text { ginger or control. The } \\
\text { first code was chosen } \\
\text { randomly (by lottery)." }\end{array}$ & $\begin{array}{l}\text { The patients were } \\
\text { randomised } \\
\text { sequentially to the two } \\
\text { groups (control and } \\
\text { intervention); that is, } \\
\text { the first patient was } \\
\text { assigned to the control } \\
\text { group and the next one } \\
\text { to the intervention } \\
\text { group, therefore } \\
\text { allocation concealment } \\
\text { inadequate. }\end{array}$ & $\begin{array}{l}\text { No placebo used therefore } \\
\text { participants and } \\
\text { researchers not blinded to } \\
\text { the intervention. Therefore } \\
\text { outcomes likely to be } \\
\text { influenced and effect of } \\
\text { intervention may have } \\
\text { been overestimated. }\end{array}$ & $\begin{array}{l}\text { Questionnaires were self } \\
\text { reported. However, } \\
\text { participants knew which } \\
\text { intervention they } \\
\text { received therefore likely } \\
\text { to influence results and } \\
\text { overestimate effect of } \\
\text { intervention. }\end{array}$ & $\begin{array}{l}22 \% \text { attrition } \\
\text { ( } 22 / 100 \text { didn't } \\
\text { complete the study). } \\
\text { Reasons given and } \\
\text { numbers per group } \\
\text { and dropout rate not } \\
\text { statistically } \\
\text { significantly } \\
\text { different between } \\
\text { the groups. Intention } \\
\text { to treat appears not } \\
\text { to have been, } \\
\text { however, } 22 \text { drop } \\
\text { outs unlikely to } \\
\text { affect results. }\end{array}$ & $\begin{array}{l}\text { No study protocol } \\
\text { identified, } \\
\text { however, all pre- } \\
\text { specified expected } \\
\text { outcomes } \\
\text { included in } \\
\text { publication as } \\
\text { intended. }\end{array}$ & $\begin{array}{l}\text { The study appears } \\
\text { to be free of other } \\
\text { sources of bias. }\end{array}$ \\
\hline Rating & & Risk of bias: Low & Risk of bias: Unclear & Risk of bias: Low & Risk of bias: & Risk of bias: Low & Risk of bias: Low & $\begin{array}{l}\text { Risk of bias: } \\
\text { Low }\end{array}$ \\
\hline Evidence & Ryan 2012 & $\begin{array}{l}\text { "Randomization was } \\
\text { stratified by CCOP site. } \\
\text { Within each site, a } \\
\text { computer-generated } \\
\text { random number table } \\
\text { with block size eight was } \\
\text { used to randomly assign } \\
\text { patients to one of four } \\
\text { treatment arms." No } \\
\text { statistically signficant } \\
\text { differences between } \\
\text { baseline characteristcs } \\
\text { suggesting randomisation } \\
\text { executed successfully. }\end{array}$ & $\begin{array}{l}\text { No description of how } \\
\text { allocation was } \\
\text { conducted. }\end{array}$ & $\begin{array}{l}\text { Placebo capsules made to } \\
\text { be identical in weight and } \\
\text { appearance, and double } \\
\text { encapsulated. }\end{array}$ & $\begin{array}{l}\text { Questionnaires given to } \\
\text { participant to complete, } \\
\text { and seeing as patients } \\
\text { blinded to intervention, } \\
\text { unlikely to have effected } \\
\text { results. }\end{array}$ & $\begin{array}{l}23 \% \text { attrition. Some } \\
\text { intention to treat } \\
\text { used where possible. } \\
\text { Numbers per group } \\
\text { and reasons given. }\end{array}$ & $\begin{array}{l}\text { Study Protocol } \\
\text { pre-registered and } \\
\text { all pre-specified } \\
\text { expected } \\
\text { outcomes } \\
\text { included in } \\
\text { publication as } \\
\text { intended. }\end{array}$ & $\begin{array}{l}\text { The study appears } \\
\text { to be free of other } \\
\text { sources of bias. }\end{array}$ \\
\hline Rating & & Risk of bias: Low & Risk of bias: Low & Risk of bias: Low & Risk of bias: Low & Risk of bias: Low & Risk of bias: Low & $\begin{array}{l}\text { Risk of bias: } \\
\text { Low }\end{array}$ \\
\hline Evidence & Zick 2009 & $\begin{array}{l}\text { "The randomization code } \\
\text { blocked by research site } \\
\text { was computer-generated } \\
\text { by the study } \\
\text { biostatistician." Baseline } \\
\text { characteristics not } \\
\text { statistically analysed, } \\
\text { however appear similar } \\
\text { suggesting randomisation } \\
\text { executed successfully. }\end{array}$ & $\begin{array}{l}\text { " All study participants } \\
\text { as well as all study } \\
\text { personnel who } \\
\text { assessed outcomes, } \\
\text { worked with study } \\
\text { data, or administered } \\
\text { tests or questionnaires } \\
\text { were unaware of the } \\
\text { randomization list or } \\
\text { treatment assignment. } \\
\text { " }\end{array}$ & $\begin{array}{l}\text { "All study participants as } \\
\text { well as all study personnel } \\
\text { who assessed outcomes, } \\
\text { worked with study data, or } \\
\text { adminis- tered tests or } \\
\text { questionnaires were } \\
\text { unaware of the } \\
\text { randomization list or } \\
\text { treatment assignment." } \\
\text { Placebo capsules made to } \\
\text { mimic ginger. }\end{array}$ & $\begin{array}{l}\text { As participants and } \\
\text { researchers blinded to } \\
\text { intervention, unlikely to } \\
\text { effect outcomes. }\end{array}$ & $\begin{array}{l}20 \% \text { attrition } \\
(33 / 162) \text {. Intention } \\
\text { to treat used. } \\
\text { Reasons per group } \\
\text { for drop outs given, } \\
\text { numbers and } \\
\text { reasons don't appear } \\
\text { drastically different } \\
\text { between groups. }\end{array}$ & $\begin{array}{l}\text { No study protocol } \\
\text { identified, } \\
\text { however, all pre- } \\
\text { specified expected } \\
\text { outcomes } \\
\text { included in } \\
\text { publication as } \\
\text { intended. }\end{array}$ & $\begin{array}{l}\text { The study appears } \\
\text { to be free of other } \\
\text { sources of bias. }\end{array}$ \\
\hline
\end{tabular}


Online Supplementary Material 3: Characteristics of Included Studies

Table 1. Included studies located in the updated search strategy $(n=13)$, examining the effect of ginger supplementation on chemotherapy-induced nausea and vomiting incidence and chemotherapy-related outcomes.

\begin{tabular}{|c|c|c|c|c|c|c|c|}
\hline Citation & $\begin{array}{l}\text { Study Design } \\
\text { \& Setting }\end{array}$ & Population & CTx Protocol & $\begin{array}{l}\text { Adjuvant } \\
\text { Therapies }\end{array}$ & $\begin{array}{l}\text { Intervention } \\
\text { Group/s (IG) }\end{array}$ & $\begin{array}{l}\text { Comparator } \\
\text { Group/s } \\
\text { (CG) }\end{array}$ & Results \\
\hline $\begin{array}{l}\text { Alparslan } 2012 \\
(24)\end{array}$ & $\begin{array}{l}\text { Design: Non- } \\
\text { RCT } \\
\text { Country: } \\
\text { Turkey } \\
\text { Study } \\
\text { Duration } \\
\text { Dates: March } \\
2011 \text { to July } \\
2011\end{array}$ & $\begin{array}{l}\text { N: } 45 \\
\text { Attrition: } 0 \% \\
\text { Females: } \mathrm{n}=15 \\
\text { Age: } 60 \% \text { were } \\
\text { 46-80 years } \\
\text { Cancer type: } \\
\text { Hematological } \\
\text { (64\% } \\
\text { leukaemia) } \\
\text { History of } \\
\text { CINV: Not } \\
\text { specified }\end{array}$ & $\begin{array}{l}\text { None specified. } \\
51 \% \text { were on } \\
\text { their first course } \\
\text { of treatment }\end{array}$ & $\begin{array}{l}\text { None } \\
\text { specified }\end{array}$ & $\begin{array}{l}\text { n: } 15 \\
\text { Type: ginger } \\
\text { tablet } \\
\text { Dose: } 1.6 \mathrm{~g} / \mathrm{d} \\
\text { (2x0.4g BD) } \\
\text { Duration: } \\
\text { Throughout } \\
\text { course of } \\
\text { treatment } \\
\text { (length } \\
\text { unspecified) }\end{array}$ & $\begin{array}{l}\text { n: } 30 \\
\text { Type: IV anti- } \\
\text { emetic (3mg } \\
\text { setron) }\end{array}$ & $\begin{array}{l}\text { CINV }^{\text {a }} \\
\text { Incidence (time point not specified): } \\
\text { - IG } n=0 / 15 ; C G \mathrm{n}=23 / 30 ; \mathbf{P}<\mathbf{0 . 0 5} \text { between groups } \\
\text { Mortality } \\
\text { Not reported }\end{array}$ \\
\hline $\begin{array}{l}\text { Arslan } 2015 \\
(25)\end{array}$ & $\begin{array}{l}\text { Design: RCT } \\
\text { Country: } \\
\text { Turkey } \\
\text { Dates: Not } \\
\text { specified }\end{array}$ & $\begin{array}{l}\text { N: } 60 \\
\text { Attrition: } 0 \% \\
\text { Female: } \mathrm{n}=60 \\
\text { Age: mean } 48.5 \\
\text { years } \\
\text { Cancer: Breast } \\
\text { cancer (stage II } \\
\text { or III) with } \\
\text { previous } \\
\text { surgical } \\
\text { treatment } \\
\text { History of } \\
\text { CINV: Yes, } \\
\text { established in } \\
\text { previous } \\
\text { Cycles. No } \\
\text { difference } \\
\text { between groups }\end{array}$ & $\begin{array}{l}\text { Drugs: } \\
\text { Anthracycline, } \\
\text { cyclophosphami } \\
\text { de, doxorubicin } \\
\text { and 5- } \\
\text { fluorouracil } \\
\text { Emetogenicity: } \\
\text { Not specified } \\
\text { Single or multi } \\
\text { day: } \\
\text { Single } \\
\text { Commencing } \\
\text { Cycle: } \geq 2^{\text {nd }} \\
\text { Cycle } \\
\text { Cycle length: } \\
\text { Not specified }\end{array}$ & $\begin{array}{l}\text { 5-HT3 } \\
\text { receptor } \\
\text { antagonist } \\
\text { (palonosetr } \\
\text { on), } \\
\text { dexamethas } \\
\text { one, an } \\
\text { antihistami } \\
\text { ne } \\
\text { (ranitidine( } \\
\text { (Day 1); } \\
\text { PO } \\
\text { aprepitant } \\
\text { (Days 1-3) }\end{array}$ & $\begin{array}{l}\mathrm{n}: 30 \\
\text { Type: } 0.5 \mathrm{~g} \\
\text { powdered } \\
\text { ginger sachet } \\
\text { mixed with } \\
\text { yoghurt } \\
\text { Dose: } 1 \mathrm{~g} / \mathrm{d} \\
\text { (0.5g BD) } \\
\text { Duration: } 3 \\
\text { days; } 1 \text { st dose } \\
30 \text { mins } \\
\text { before CTx; } \\
\text { for } 2 \text { Cycles }\end{array}$ & $\begin{array}{l}\text { n: } 30 \\
\text { Type: } \\
\text { Standard care } \\
\text { (no ginger) }\end{array}$ &  \\
\hline
\end{tabular}




\begin{tabular}{|c|c|c|c|c|c|c|c|}
\hline $\begin{array}{l}\text { Bossi } 2017 \\
\text { (39) }\end{array}$ & $\begin{array}{l}\text { Design: } \\
\text { Double-blind } \\
\text { placebo- } \\
\text { controlled } \\
\text { randomised } \\
\text { trial } \\
\text { Country: Italy } \\
\text { Data } \\
\text { Collection } \\
\text { Dates: June } \\
2013 \text { to April } \\
2015\end{array}$ & 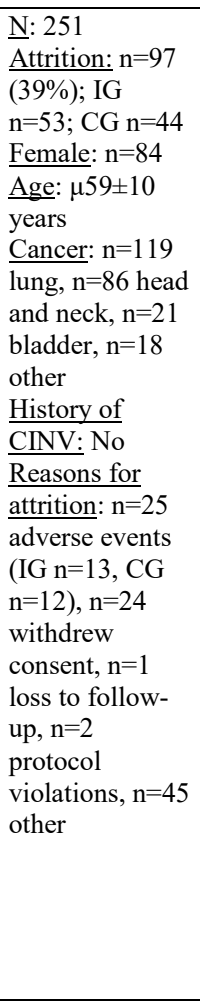 & $\begin{array}{l}\text { Drugs: } \\
\text { Cisplatin }>50 \mathrm{~m} \\
\mathrm{~g} / \mathrm{m}^{2} \text { and other } \\
\text { unspecified } \\
\text { Emetogenicity: } \\
\text { High } \\
\text { Single or multi } \\
\text { day: Single } \\
\text { Commencing } \\
\text { Cycle: Cycle } 1 \\
\text { Cycle length: } \\
\mathrm{n}=140 \text { were } 21 \\
\text { days. } \mathrm{N}=4 \text { were } \\
28 \text { days }\end{array}$ & $\begin{array}{l}\text { Aprepitant } \\
\text { and 5-HT3 } \\
\text { receptor } \\
\text { antagonist } \\
\text { (Day 1); } \\
\text { dexamethas } \\
\text { one (Days } \\
\text { 1-4) }\end{array}$ & $\begin{array}{l}\text { n: } 125 \\
\text { Type: Softel } \\
\text { capsule with } \\
\text { sunflower oil } \\
(110 \mathrm{mg}) \text { and } \\
\text { zingiber } \\
\text { officinalis } \\
\text { standardised } \\
\mathrm{CO} 2 \\
\text { supercritical } \\
\text { extract } \\
\text { (40mg); } \\
\text { minimum } \\
\text { Gingerols } \\
16 \mathrm{mg}, \text { min } \\
\text { Shogaol } \\
1.12 \mathrm{mg} \\
\text { Dose: } \\
0.16 \mathrm{~g} / \mathrm{d} \\
\text { (2x0.04g } \\
\text { BD). } \\
\text { Duration: } \\
42-56 \text { days } \\
\text { covering n=2 } \\
\text { Cycles, } \\
\text { commenced } \\
\text { on Day 2 of } \\
\text { each 21-28- } \\
\text { day Cycle }\end{array}$ & $\begin{array}{l}\text { n: } 126 \\
\text { Type: } \\
\text { Placebo of } \\
\text { softgel } \\
\text { capsule } \\
150 \mathrm{mg} \\
\text { sunflower oil }\end{array}$ & 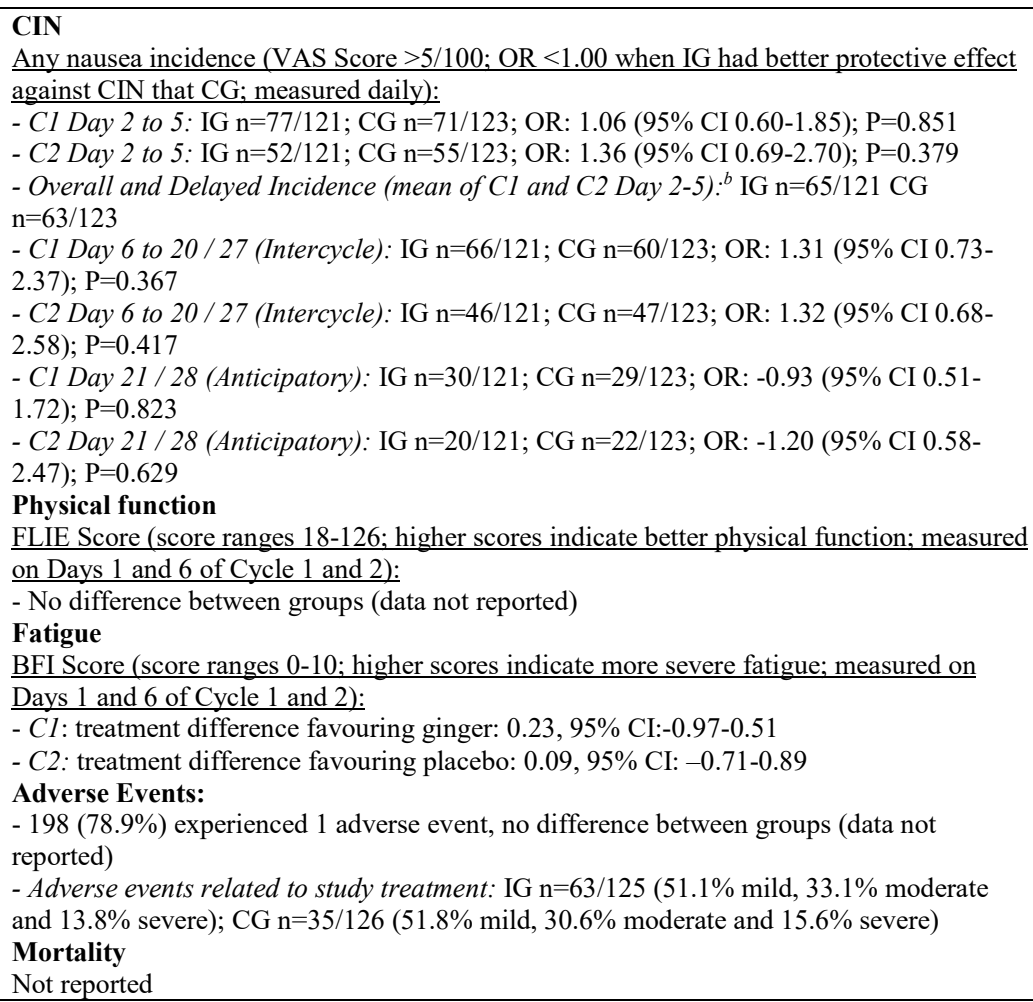 \\
\hline $\begin{array}{l}\text { Danwilai } \\
2017 \text { (35) }\end{array}$ & $\begin{array}{l}\text { Design: Pilot } \\
\text { double-blind } \\
\text { placebo-RCT } \\
\text { Country: } \\
\text { Thailand } \\
\text { Recruitment } \\
\text { Dates: } \\
\text { September } \\
2012 \text { to July } \\
2013\end{array}$ & $\begin{array}{l}\text { N: } 50 \\
\text { Attrition: } \mathrm{n}=7 \\
\text { (14\%); IG } \mathrm{n}=6 \text {; } \\
\text { CG n=1 } \\
\text { Female: } \mathrm{n}=43 \\
\text { Age: } 52.4 \pm 9.1 \\
\text { years } \\
\text { Cancer: Breast } \\
\text { (91\%), ovarian } \\
\text { and lung } \\
\text { History of } \\
\text { CINV: No } \\
\text { Reasons for } \\
\text { attrition: } \mathrm{n}=3 \\
\text { withdrew } \\
\text { consent, } \mathrm{n}=1 \\
\text { unable to } \\
\text { swallow } \\
\text { capsule, } \mathrm{n}=3 \\
\text { transferred to } \\
\text { different site }\end{array}$ & $\begin{array}{l}\text { Drugs: Mostly } \\
\text { anthracycline } \\
\text { based regimen } \\
\text { or some } \\
\text { platinum based } \\
\text { regimen. } \\
\text { Emetogenicity: } \\
\text { Moderate or } \\
\text { high } \\
\text { Single or multi } \\
\text { day: Not } \\
\text { specified } \\
\text { Commencing } \\
\text { Cycle: Cycle } 1 \\
\text { Cycle length: } \\
\text { Not specified }\end{array}$ & $\begin{array}{l}\text { Dexametha } \\
\text { sone and } \\
\text { ondansetro } \\
\text { n prior to } \\
\text { CTx } \\
\text { administrati } \\
\text { on. Rescue } \\
\text { anti- } \\
\text { emetics at } \\
\text { any time if } \\
\text { needed. } \\
\text { Those } \\
\text { given } \\
\text { aprepitants } \\
\text { not eligible } \\
\text { for } \\
\text { inclusion }\end{array}$ & $\begin{array}{l}\text { n: } 25 \\
\text { Type: } \\
\text { Capsule with } \\
0.005 \mathrm{~g} \\
\text { standardised } \\
\text { 6-gingerol } \\
\text { (1.4\% w/w of } \\
\text { ginger } \\
\text { extract); } \\
\text { binder; } \\
\text { thickening } \\
\text { agent } \\
\text { Dose: } 0.02 \mathrm{~g} / \mathrm{d} \\
\text { (2x 0.005g } \\
\text { BD). } \\
\text { Duration: } \\
\text { from } 3 \text { days } \\
\text { prior to CTx } \\
\text { through to the } \\
4^{\text {th }} \text { Cycle }\end{array}$ & $\begin{array}{l}\text { n }: 25 \\
\text { Type: placebo } \\
\text { capsule with } \\
\text { binder, } \\
\text { thickening } \\
\text { agent and } \\
\text { matched } \\
\text { weight }\end{array}$ & $\begin{array}{l}\text { Adverse Events: } \\
\text { - Withdrawals: IG 6; CG } 1 \\
\text { - Toxicity ( } \text { withdrawn due to unacceptable toxicity): IG } \mathrm{n}=0 / 25 ; \mathrm{CG} \text { not specified } \\
\text { - Adverse events reasonably related to study treatment: } \mathrm{IG} \mathrm{n}=0 / 19 ; \mathrm{CG} \text { not specified } \\
\text { Mortality } \\
\text { Not reported }\end{array}$ \\
\hline
\end{tabular}




\begin{tabular}{|c|c|c|c|c|c|c|c|}
\hline $\begin{array}{l}\text { Konmun } \\
2017(36)\end{array}$ & $\begin{array}{l}\text { Design: } \\
\text { Double-blind } \\
\text { placebo- } \\
\text { controlled } \\
\text { randomised } \\
\text { trial } \\
\text { Country: } \\
\text { Thailand } \\
\text { Recruitment } \\
\text { Dates: } \\
\text { January } 2012 \\
\text { to July 2013 }\end{array}$ & $\begin{array}{l}\text { N: } 88(\mathrm{n}=94 \\
\text { randomised but } \\
\mathrm{n}-88 \\
\text { commenced } \\
\text { study) } \\
\text { Attrition: } 7 \\
\text { (8\%) } \\
\text { Female: } \mathrm{n}=75 \\
\text { Age: median } 53 \\
\text { (range } 19-81) \\
\text { years } \\
\text { Cancer: Solid } \\
\text { tumors; breast } \\
\text { cancer (72\%); } \\
\text { with prior } \\
\text { surgical } \\
\text { removal } \\
\text { History of } \\
\text { CINV: No } \\
\text { Reasons for } \\
\text { attrition: } \mathrm{n}=1 \\
\text { protocol } \\
\text { violation, } \mathrm{n}=6 \\
\text { withdrew } \\
\text { consent }\end{array}$ & $\begin{array}{l}\text { Drugs: } 68 \% \\
\text { anthracycline- } \\
\text { based, } 21 \% \\
\text { platinum-based. } \\
\text { Emetogenicity: } \\
\text { Moderate or } \\
\text { high } \\
\text { Single or multi } \\
\text { day: Not } \\
\text { specified } \\
\text { Commencing } \\
\text { Cycle: Cycle } 1 \\
\text { Cycle length: } \\
\text { Not specified }\end{array}$ & $\begin{array}{l}\text { Ondansetro } \\
\mathrm{n} \text { and } \\
\text { dexamethas } \\
\text { one prior to } \\
\text { CTx (IV } \\
\text { Day 1, PO } \\
\text { Days 1-4); } \\
\text { PO } \\
\text { Metoclopra } \\
\text { mide (days } \\
\text { 2-4). Those } \\
\text { given } \\
\text { aprepitants } \\
\text { not eligible } \\
\text { for } \\
\text { inclusion }\end{array}$ & $\begin{array}{l}\mathrm{n}: 46 \\
\text { Type: } \\
\text { Capsule with } \\
0.005 \mathrm{~g} \\
\text { standardised } \\
6 \text {-gingerol } \\
(1.4 \% \mathrm{w} / \mathrm{w} \text { of } \\
\text { ginger } \\
\text { extract) } \\
\text { Dose: } 0.02 \mathrm{~g} / \mathrm{d} \\
\text { (2x0.005g } \\
\text { BD) } \\
\text { Duration: } \\
\text { from } \\
3 \text { days prior } \\
\text { to CTx for at } \\
\text { least } 3 \text { Cycles } \\
\text { (through to } 12 \\
\text { weeks of } \\
\text { treatment or } \\
\text { completion of } \\
\text { planned CTx) }\end{array}$ & $\begin{array}{l}\text { n: } 42 \\
\text { Type: } \\
\text { Placebo - } \\
\text { capsules with } \\
\text { diluents/binde } \\
\text { r and } \\
\text { thickening } \\
\text { agent }\end{array}$ & 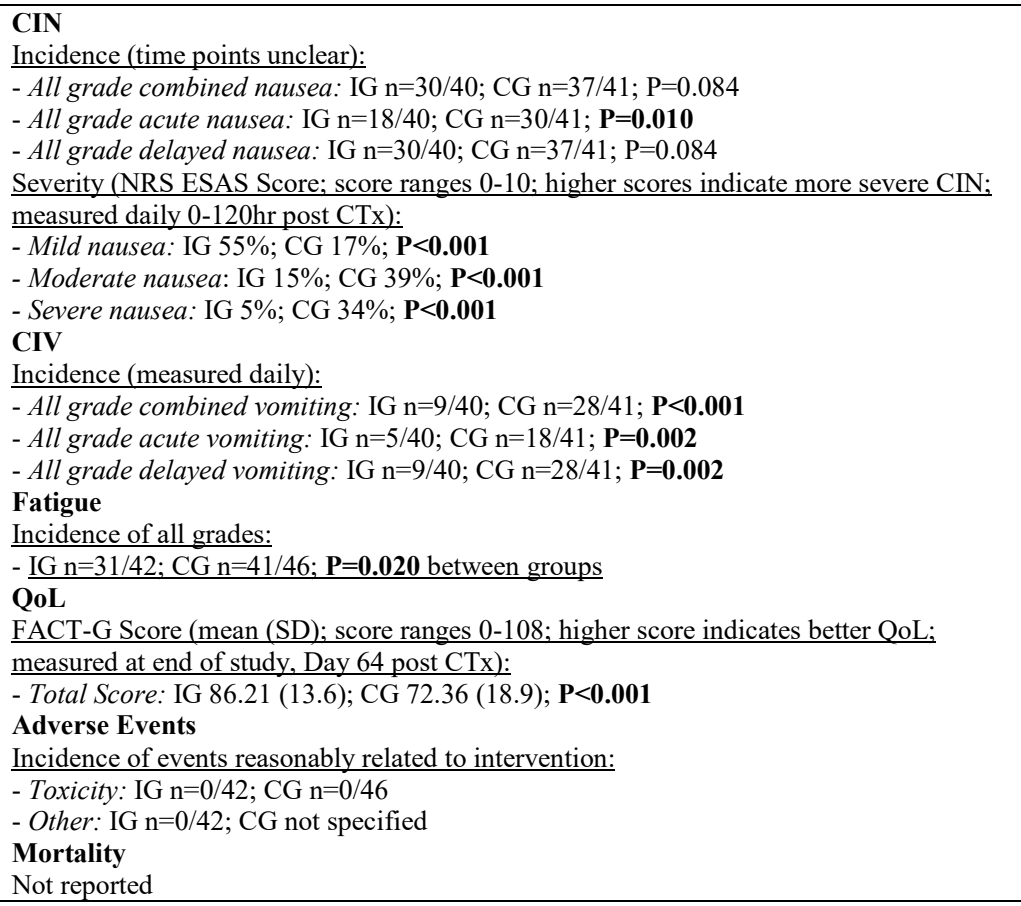 \\
\hline Li 2018 (40) & $\begin{array}{l}\text { Design: } \\
\text { Double-blind } \\
\text { placebo-RCT } \\
\text { Country: } \\
\text { China } \\
\text { Recruitment } \\
\text { Dates: June } \\
\text { 2016 to } \\
\text { March 2017 }\end{array}$ & $\begin{array}{l}\text { N: } 146 \\
\text { Attrition: } n=6 \\
(4 \%)(I G n=2, \\
\text { IG } n=4) \\
\text { Female: } n=40 \\
\text { Age: IG } 57 \pm 7- \\
8 \text { years } \\
\text { Cancer: lung } \\
\text { History of } \\
\text { CINV: IG } 59 \%, \\
\text { CG } 66 \% \\
\text { Reasons for } \\
\text { attrition: self- } \\
\text { reported lack of } \\
\text { compliance } n=6\end{array}$ & $\begin{array}{l}\text { Drugs: Cisplatin } \\
68 \%, \\
\text { carboplatin } \\
26 \% \text {, oxaliplatin } \\
6 \% \\
\text { Emetogenicity: } \\
\text { Single or multi } \\
\text { day: not } \\
\text { specified } \\
\text { Commencing } \\
\text { Cycle: any } \\
\text { Cycle length: } \\
\text { any }\end{array}$ & $\begin{array}{l}\text { Standard } \\
\text { antiemetic } \\
\text { therapy } \\
\left(5 \mathrm{HT}_{3}\right. \\
\mathrm{RA}^{\prime} \text { 's). } 66 \% \\
\text { had } \\
\text { aprepitants }\end{array}$ & $\begin{array}{l}\text { n: } 71 \\
\text { Type: } 0.25 \mathrm{~g} \\
\text { capsule with } \\
\text { standardised } \\
\text { dry ginger } \\
\text { root powder } \\
\text { with } 0.013 \mathrm{~g} \\
(5 \%) \\
\text { gingerols } \\
\text { Dose: } 0.5 \mathrm{~g} / \mathrm{d} \\
\text { (2x0.25g BD) } \\
\text { Duration: for } \\
5 \text { days } \\
\text { starting on } \\
\text { first day of } \\
\text { CTx }\end{array}$ & $\begin{array}{l}\text { n: } 69 \\
\text { Type: } 0.25 \mathrm{~g} \\
\text { placebo } \\
\text { capsule with } \\
\text { corn starch }\end{array}$ & 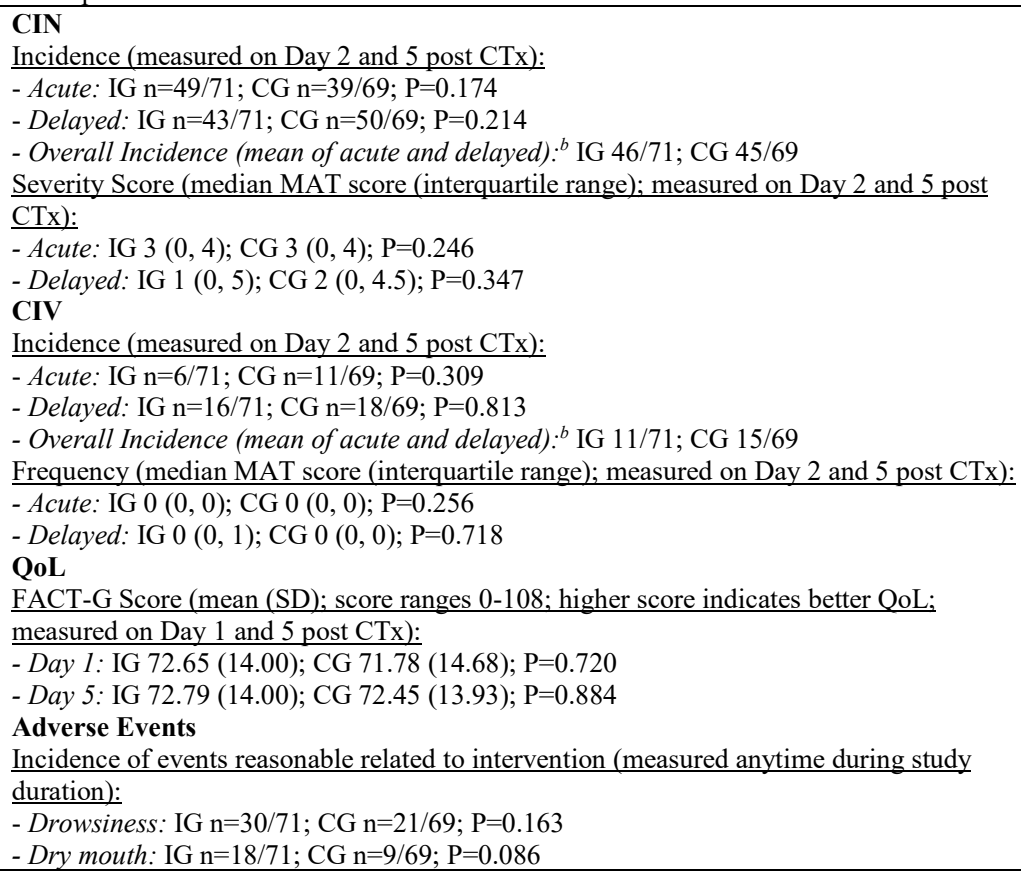 \\
\hline
\end{tabular}




\begin{tabular}{|c|c|c|c|c|c|c|c|}
\hline & & & & & & & $\begin{array}{l}\text { - Heartburn: } \mathrm{IG} \mathrm{n}=6 / 71 ; \mathrm{CG} \mathrm{n}=3 / 69 ; \mathrm{P}=0.494 \\
\text { - Flushing: } \mathrm{IG} \mathrm{n}=11 / 71 ; \mathrm{CG} \mathrm{n}=5 / 69 ; \mathrm{P}=0.184 \\
\text { Mortality } \\
\text { Not reported }\end{array}$ \\
\hline $\begin{array}{l}\text { Marx } 2017 \\
(41)\end{array}$ & $\begin{array}{l}\text { Design: } \\
\text { Double-blind } \\
\text { placebo-RCT } \\
\text { Country: } \\
\text { Australia } \\
\text { Recruitment } \\
\text { Dates: March } \\
\text { 2014 to } \\
\text { February } \\
2015\end{array}$ & $\begin{array}{l}\text { N: } 51 \\
\text { Attrition: } \mathrm{n}=17 \\
(33 \%) ; \mathrm{n}=9 \text { IG; } \\
\mathrm{n}=8 \mathrm{CG} \\
\text { Female: } \mathrm{n}=32 \\
\text { Age: } 58 \pm 12 \\
\text { years } \\
\text { Cancer: } 37 \% \\
\text { colon, } 26 \%, \\
\text { breast, } 22 \% \\
\text { lymphoma, } \\
\text { other. } \\
\text { History of } \\
\text { CINV: No } \\
\text { Reasons for } \\
\text { attrition: } \mathrm{n}=4 \\
\text { adverse event } \\
\text { (n=3 CG, } \mathrm{n}=1 \\
\text { IG), } \mathrm{n}=3 \\
\text { nausea/vomiting } \\
\text {, }=1 \text { loss to } \\
\text { follow-up, } \mathrm{n}=9 \\
\text { withdrew } \\
\text { consent }\end{array}$ & $\begin{array}{l}\text { Drugs: Not } \\
\text { specified } \\
\text { Emetogenicity: } \\
85 \% \text { moderate, } \\
16 \% \text { high } \\
\text { Single or multi } \\
\text { day: Single day } \\
\text { Commencing } \\
\text { Cycle: Cycle } 1 \\
\text { Cycle length: } \\
\text { Not specified }\end{array}$ & $\begin{array}{l}\text { Standard } \\
\text { anti- } \\
\text { emetics } \\
\text { prescribed } \\
\text { by } \\
\text { physician } \\
\text { not } \\
\text { specified }\end{array}$ & $\begin{array}{l}\text { n: } 24 \\
\text { Type: } 0.3 \mathrm{~g} \\
\text { capsule } \\
\text { standardised } \\
\text { ginger extract } \\
\text { with } 5 \% \\
(0.015 \mathrm{~g}) \\
\text { gingerols } \\
\text { Dose: } 1.2 \mathrm{~g} \\
\text { (0.3g QID) } \\
\text { Duration: } 5 \\
\text { days starting } \\
\text { on the day of } \\
\text { CTx from } \\
\text { Cycle } 1-3\end{array}$ & $\begin{array}{l}\mathrm{n}: 27 \\
\text { Type: } 0.3 \mathrm{~g} \\
\text { placebo } \\
\text { capsule with } \\
\text { inert filler }\end{array}$ & 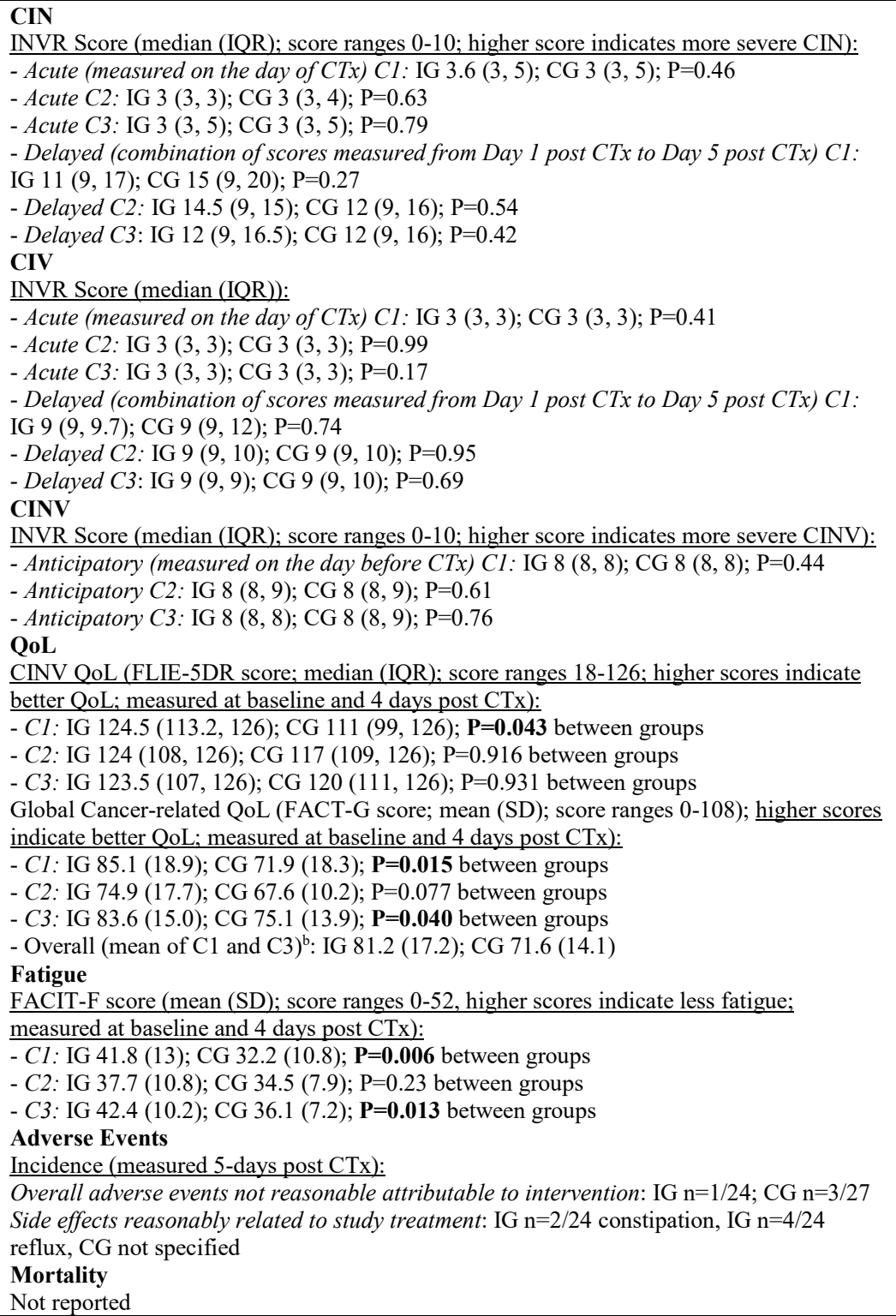 \\
\hline
\end{tabular}




\begin{tabular}{|c|c|c|c|c|c|c|c|}
\hline $\begin{array}{l}\text { Montazeri } \\
2013(30)\end{array}$ & $\begin{array}{l}\text { Design: } \\
\text { Cross-over } \\
\text { double } \\
\text { blinded, } \\
\text { placebo RCT } \\
\text { Country: } \\
\text { Unclear, } \\
\text { possibly Iran } \\
\text { Data } \\
\text { Collection } \\
\text { Dates: } \\
\text { September } \\
\text { 2006 to } \\
\text { March } 2007\end{array}$ & $\begin{array}{l}\mathrm{N}: 44 \\
\text { Attrition: } \mathrm{n}=13 \\
\text { (30\%) during } \\
\text { second } \\
\text { intervention } \\
\text { period, unclear } \\
\text { from which } \\
\text { groups. } \\
\text { Female: } \mathrm{n}=18 \\
\text { Age: mean } 50.3 \\
\pm 3.1 \text { years } \\
\text { Cancer: mainly } \\
\text { oesophagus } \\
\text { History of } \\
\text { CINV: Yes } \\
\text { Reason for } \\
\text { attrition: } \mathrm{n}=3 \\
\text { death, } \mathrm{n}=7 \\
\text { protocol } \\
\text { violation, } \mathrm{n}=2 \\
\text { vomiting, } \mathrm{n}=1 \\
\text { loss to follow- } \\
\text { up }\end{array}$ & $\begin{array}{l}\text { Drugs: Cisplatin } \\
\text { with or without } \\
\text { other } \\
\text { unspecified } \\
\text { CTx agents } \\
\text { (most } \\
\text { commonly } \\
\text { fluorouracil 5) } \\
\text { Emetogenicity: } \\
\text { Not specified } \\
\text { Single or multi } \\
\text { day: Not } \\
\text { specified } \\
\text { Commencing } \\
\text { Cycle: After } \\
\text { Cycle }>1, \text { not } \\
\text { specified }\end{array}$ & $\begin{array}{l}\text { Grainestron } \\
\text { e and } \\
\text { dexamthaso } \\
\text { ne and } \\
\text { metoclopra } \\
\text { mide if } \\
\text { requested }\end{array}$ & $\begin{array}{l}\text { n: Unclear; } \\
\text { possibly } 37 \\
\text { Type: } 0.25 \mathrm{~g} \\
\text { ginger } \\
\text { powder } \\
\text { capsule } \\
\text { Dose: } 1 \mathrm{~g} / \mathrm{d} \\
\text { (2x } 0.25 \mathrm{~g} \\
\text { BD) } \\
\text { Duration: for } \\
\text { one Cycle (at } \\
\text { least } 28 \text { days) } \\
\text { before } \\
\text { crossing over }\end{array}$ & $\begin{array}{l}\text { n: Unclear; } \\
\text { possibly } 37 \\
\text { Type: } 0.25 \mathrm{~g} \\
\text { placebo } \\
\text { capsule with } \\
\text { chickpea } \\
\text { flour }(2 \mathrm{x} \\
0.25 \mathrm{~g} \mathrm{BD})\end{array}$ & $\begin{array}{l}\text { CINV } \\
\text { Severity (Strain Tools of Severity; score ranges } 0-10 \text {; higher score indicates more severe } \\
\text { CIN; measured } 7,8,9,10 \text { and } 24 \text { hours post CTx): } \\
\text { - C1: in the } 7 \text { th, 8th, } 9 \text { th, } 10 \text { th and } 24 \text { th hours post CTx, severity reduced } 9.1 \%, 9.1 \%, 9.1 \% \\
4.6 \% \text { and } 4.7 \% \text { more in IG than CG, respectively. } \\
-C 2: \text { In the } 8 \text { th, } 9 \text { th, } 10 \text { th and } 24 \text { th hours post CTx, severity reduced } 4 \%, 6.3 \%, 6.7 \% \text { and } \\
8.3 \% \text { more in the CG than IG, respectively. There was no significant difference in the } 7 \text { th } \\
\text { hour post CTx } \\
\text { Mortality } \\
-n=3 / 37\end{array}$ \\
\hline $\begin{array}{l}\text { Muthia } 2013 \\
\text { (26) }\end{array}$ & $\begin{array}{l}\text { Design: } \\
\text { Control time } \\
\text { series } \\
\text { Country: } \\
\text { Indonesia } \\
\text { Data } \\
\text { Collection } \\
\text { Dates: April } \\
2013 \text { to May } \\
2013\end{array}$ & $\begin{array}{l}\text { N: } 20 \\
\text { Attrition: } \mathrm{n}=0 \\
\text { Female: } \mathrm{n}=20 \\
\text { Age: unclear. } \\
\text { Cancer: Breast } \\
\text { History of } \\
\text { CINV: Unclear }\end{array}$ & $\begin{array}{l}\text { Drugs: } \\
\text { Cyclophospham } \\
\text { ide, } \\
\text { Adriamycin-5- } \\
\text { fluorouracil } \\
\text { Emetogenicity: } \\
\text { Not specified } \\
\text { Single or multi } \\
\text { day: Not } \\
\text { specified } \\
\text { Commencing } \\
\text { Cycle: Not } \\
\text { specified } \\
\text { Cycle length: } \\
\text { Not specified }\end{array}$ & $\begin{array}{l}\text { Ondasentro } \\
\mathrm{n} \text { and } \\
\text { dexamethas } \\
\text { one }\end{array}$ & $\begin{array}{l}\text { n: } 10 \\
\text { Type: Self- } \\
\text { prepared } \\
\text { ginger } \\
\text { infusion }(10 \mathrm{~g} \\
\text { fresh Zingiber } \\
\text { officanale } \\
\text { varietas } \\
\text { rubrum } \\
\text { [pared and } \\
\text { grated] + } \\
100 \mathrm{~mL} \text { water } \\
\text { to make. } \\
\text { Infused for } 15 \\
\text { minutes } \\
\text { starting at } \\
90^{\prime} \mathrm{C} \text {, strained } \\
\text { and diluted to } \\
150 \mathrm{ml} \text {. } \\
\text { Dose: } 1 \text { serve } \\
\text { [unclear if } \\
15 \mathrm{ml} \text { or } \\
150 \mathrm{ml}] \mathrm{x} 3 / \mathrm{d} \\
\text { Duration: Not } \\
\text { specified, } \\
\text { from the } 2^{\text {nd }} \\
\text { day after CTx }\end{array}$ & $\begin{array}{l}\text { n: } 10 \\
\text { Type: no } \\
\text { intervention / } \\
\text { standard care }\end{array}$ & 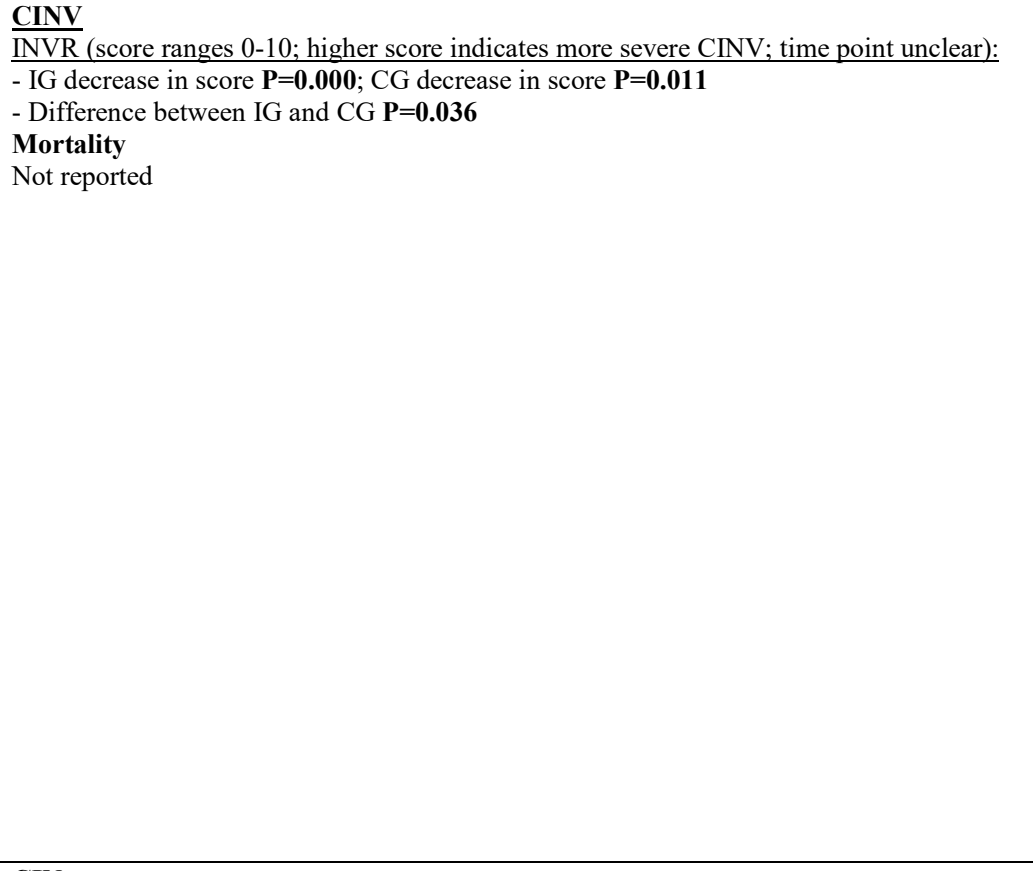 \\
\hline $\begin{array}{l}\text { Sanaati } 2016 \\
(31)\end{array}$ & $\begin{array}{l}\text { Design: } \\
\text { Double blind } \\
\text { RCT } \\
\text { Country: Iran }\end{array}$ & $\begin{array}{l}\mathrm{N}: 43 \\
\text { Attrition: } \mathrm{n}=13 \\
(30 \%) ; \mathrm{n}=7 \mathrm{IG} \\
\mathrm{n}=5 \mathrm{CG}\end{array}$ & $\begin{array}{l}\text { Drugs: Not } \\
\text { specified } \\
\text { Emetogenicity: } \\
\text { Not specified }\end{array}$ & $\begin{array}{l}\text { Dexametha } \\
\text { sone, } \\
\text { metoclopra } \\
\text { mide and } \\
\text { aprepitant }\end{array}$ & $\begin{array}{l}\text { n: } 23 \\
\text { Type: } 0.5 \mathrm{~g} \\
\text { capsule } \\
\text { powdered } \\
\text { ginger root }\end{array}$ & $\begin{array}{l}\text { n: } 20 \\
\text { Type: } \\
\text { Standard Care } \\
\text { (no ginger) }\end{array}$ & $\begin{array}{l}\text { CIN } \\
\text { Incidence (self-designed tool; measured every night from } 5 \text { days before to } 5 \text { days after } \\
\text { CTx): } \\
\text { - Mean difference between groups } 1.5 \text { (S.E. } 0.58 \text { ); } \mathbf{P}=\mathbf{0 . 0 0 6} \\
\text { CIV }\end{array}$ \\
\hline
\end{tabular}




\begin{tabular}{|c|c|c|c|c|c|c|c|}
\hline & $\begin{array}{l}\text { Recruitment } \\
\text { Dates: May } \\
2013 \text { to June } \\
2014\end{array}$ & $\begin{array}{l}\text { Female: Not } \\
\text { specified } \\
\text { Age: range 20- } \\
\text { 60 years } \\
\text { Cancer: Breast } \\
\text { History of } \\
\text { CINV: Yes } \\
\text { Reasons for } \\
\text { attrition: CTx } \\
\text { cancelled, } \\
\text { withdrew, not } \\
\text { completing data } \\
\text { collection, death } \\
\text { (numbers per } \\
\text { group unclear) }\end{array}$ & $\begin{array}{l}\frac{\text { Single or multi }}{\text { day: Single }} \\
\underline{\text { Commencing }} \\
\frac{\text { Cycle: }: \geq \text { Cycle }}{2} \\
\text { Cycle length: } \\
\geq 2 \text { weeks }\end{array}$ & $\begin{array}{l}\text { (DMA) } \\
\text { capsule }\end{array}$ & $\begin{array}{l}\text { Dose: } \\
1 \mathrm{~g} / \mathrm{d}(0.5 \mathrm{~g} \\
\text { BD) } \\
\text { Duration: } 10 \\
\text { days (from } 5 \\
\text { days before to } \\
5 \text { days after } \\
\text { CTx) }\end{array}$ & & 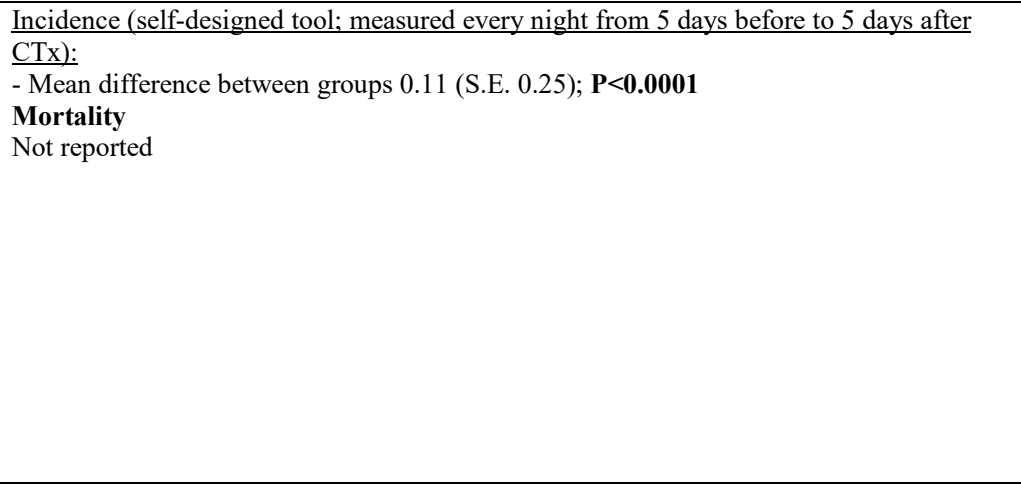 \\
\hline $\begin{array}{l}\text { Shokri } 2017 \\
\text { (32) }\end{array}$ & $\begin{array}{l}\text { Design: } \\
\text { Double blind, } \\
\text { placebo RCT } \\
\text { Country: Iran } \\
\text { Data } \\
\text { Collection } \\
\text { and Analysis } \\
\text { Dates: } \\
\begin{array}{l}\text { October } 2014 \\
\text { to February } \\
2015\end{array}\end{array}$ & $\begin{array}{l}\text { N: } 49 \\
\text { Attrition: } \mathrm{n}=0 \\
\text { Female: } \mathrm{n}=49 \\
\text { Age: mean } 53 \pm \\
11-16 \text { years. } \\
\text { Cancer: Stage I } \\
\text { to III ovarian } \\
\text { cancer who had } \\
\text { undergone } \\
\text { cytoreductive } \\
\text { surgery } \\
\text { History of } \\
\text { CINV: Not } \\
\text { specified }\end{array}$ & $\begin{array}{l}\text { Drugs: } \\
\text { Carboplatin and } \\
\text { paclitaxel } \\
\text { Emetogenicity: } \\
\text { Not specified } \\
\text { Single or multi } \\
\text { day: Not } \\
\text { specified } \\
\text { Commencing } \\
\text { Cycle: Not } \\
\text { specified } \\
\text { Cycle length: } 21 \\
\text { days }\end{array}$ & $\begin{array}{l}\text { Not } \\
\text { specified }\end{array}$ & $\begin{array}{l}\mathrm{n}: 20 \\
\text { Type: } 1 \mathrm{~g} \\
\text { capsule } \\
\text { Dose: } 2 \mathrm{~g} / \mathrm{d} \\
\text { (1g BD) } \\
\text { Duration: for } \\
\text { 6 Cycles }\end{array}$ & $\begin{array}{l}\text { n: } 29 \\
\text { Type: } 1 \mathrm{~g} \\
\text { placebo } \\
\text { capsule } \\
\text { Dose: } 2 \mathrm{~g} / \mathrm{d} \\
\text { (1g BD) } \\
\text { Duration: for } \\
\text { 6Cycles }\end{array}$ & $\begin{array}{l}\text { CINV } \\
\text { Incidence (time point not specified): } \\
\text { - IG } \mathrm{n}=8 / 20 ; \mathrm{CG} \mathrm{n}=14 / 29 ; \mathrm{P}=0.57 \text { between groups } \\
\text { Adverse Events } \\
\text { Incidence (measured at the end of treatment up to } 12 \text { months after baseline): } \\
\text { - hematologic, renal and digestive complications (unspecified): } \mathrm{IG} \mathrm{n}=10 / 20 ; \mathrm{CG} \mathrm{n}=21 / 29 ; \\
\mathrm{P}=0.11 \text { between groups } \\
\text { - weight loss: } \mathrm{IG} \mathrm{n}=1 / 20 ; \mathrm{CG} \mathrm{n}=1 / 29 ; \mathrm{P}=0.66 \text { between groups } \\
\text { - peripheral neuropathy: } \mathrm{IG} \mathrm{n}=3 / 20 ; \mathrm{CG} \mathrm{n}=5 / 29 ; \mathrm{P}=0.58 \text { between groups } \\
\text { - } \text { bone marrow depression: } \mathrm{IG} \mathrm{n}=2 / 20 ; \mathrm{CG} \mathrm{n}=2 / 29 ; \mathrm{P}=0.54 \text { between groups } \\
\text { - transient cortical blindness: } \mathrm{IG} \mathrm{n}=1 / 20 ; \mathrm{CG} \mathrm{n}=0 / 29 ; \mathrm{P}=0.41 \text { between groups } \\
\text { - peripheral neuropathy: } \mathrm{IG} \mathrm{n}=3 / 20 ; \mathrm{CG} \mathrm{n}=5 / 29 ; \mathrm{P}=0.58 \text { between groups } \\
\text { Mortality } \\
\text { Incidence (during } 12-\text { month follow up period): } \\
\text { - IG } \mathrm{n}=2 / 20 ; \mathrm{CG} \mathrm{n}=3 / 29 ; \mathrm{P}=0.68 \text { between groups }\end{array}$ \\
\hline $\begin{array}{l}\text { Thamlikitkul } \\
2017 \text { (37) }\end{array}$ & 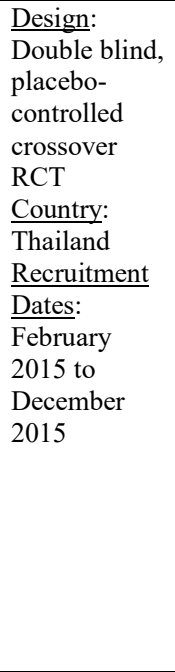 & $\begin{array}{l}\text { N: } 34 \\
\text { Attrition: } 0 \\
\text { Female: } n=34 \\
\text { Age: mean } 49 \\
\text { (range 32-68) } \\
\text { years } \\
\text { Cancer: Breast } \\
\text { History of } \\
\text { CINV: Yes }\end{array}$ & $\begin{array}{l}\text { Drugs: } \\
\text { Cyclophospham } \\
\text { ide and } \\
\text { doxorubicin } \\
\text { Emetogenicity: } \\
\text { High } \\
\text { Single or multi } \\
\text { day: Not } \\
\text { specified } \\
\text { Commencing } \\
\text { Cycle: } \\
\text { Cycle } 2 \\
\text { Cycle length: } \\
\text { Not specified }\end{array}$ & $\begin{array}{l}\text { Ondansetro } \\
\mathrm{n} \text { and } \\
\text { dexamethas } \\
\text { one and } \\
\text { rescue } \\
\text { domperidon } \\
\text { e or } \\
\text { metoclopra } \\
\text { mide } \\
\text { if needed }\end{array}$ & $\begin{array}{l}\text { n: } 34 \\
\text { Type: } \\
\text { capsuled dry } \\
\text { ginger } \\
\text { powder } \\
\text { Dose: } 1 \mathrm{~g} \\
(0.5 \mathrm{~g} B \mathrm{BD}) \\
\text { Duration: } 5 \\
\text { days starting } \\
\text { on Day } 1 \text { of } \\
\text { CTx for one } \\
\text { Cycle then } \\
\text { crossed over } \\
\text { to second } \\
\text { group for the } \\
\text { next Cycle }\end{array}$ & $\begin{array}{l}\text { n: } 34 \\
\text { Type: } \\
\text { Placebo } \\
\text { capsule } \\
\text { Dose: } 0.5 \mathrm{~g} \\
\text { BD } \\
\text { Duration: } 5 \\
\text { days starting } \\
\text { on Day } 1 \text { of } \\
\text { CTx for one } \\
\text { Cycle then } \\
\text { crossed over } \\
\text { to second } \\
\text { group for the } \\
\text { next Cycle }\end{array}$ & 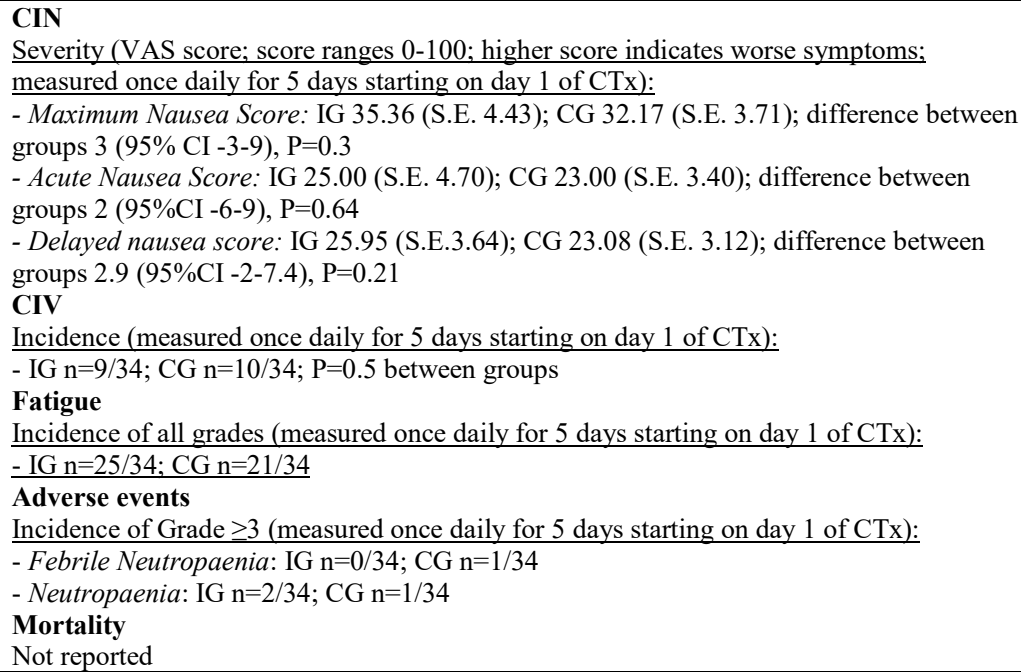 \\
\hline
\end{tabular}




\begin{tabular}{|c|c|c|c|c|c|c|c|}
\hline $\begin{array}{l}\text { Yekta } 2012 \\
\text { (33) }\end{array}$ & $\begin{array}{l}\text { Design: } \\
\text { Double blind, } \\
\text { placebo-RCT } \\
\text { Country: Iran } \\
\text { Study } \\
\text { Duration } \\
\text { Dates: July } \\
\text { 2009 to } \\
\text { December } \\
2009\end{array}$ & $\begin{array}{l}\mathrm{N}: 98 \\
\text { Attrition: } \mathrm{n}=18 \\
(18 \%) ; \text { unclear } \\
\text { from which } \\
\text { groups. } \\
\text { Female: } \mathrm{n}=80 \\
\text { Cancer: Breast } \\
\text { History of } \\
\text { CINV: Yes } \\
\text { Reasons for } \\
\text { attrition: } \\
\text { cancelled CTx, } \\
\text { withdrew, did } \\
\text { not complete } \\
\text { data collection, } \\
\text { death. Unclear } \\
\text { numbers from } \\
\text { each group. }\end{array}$ & $\begin{array}{l}\text { Drugs: Not } \\
\text { specified } \\
\text { Emetogenicity: } \\
84 \% \text { moderate } \\
\text { to high } \\
\text { Single or multi } \\
\text { day: Single } \\
\text { Commencing } \\
\text { Cycle: } \\
\geq \text { Cycle } 2 \\
\text { Cycle length: } \geq \\
2 \text { weeks }\end{array}$ & $\begin{array}{l}\text { Kytril or } \\
\text { Granisetron } \\
\text { hydrochlori } \\
\text { de tablets } \\
\text { and } \\
\text { dexamethas } \\
\text { one }\end{array}$ & $\begin{array}{l}\mathrm{n}: 40 \\
\text { Type: } 0.25 \mathrm{~g} \\
\text { ginger root } \\
\text { capsules, dry } \\
\text { ginger root } \\
\text { (5.38mg 6- } \\
\text { gingerol, } \\
1.8 \mathrm{mg} 8- \\
\text { gingerol, } \\
4.19 \mathrm{mg} 10- \\
\text { gingerol, } \\
0.92 \mathrm{mg} \text { 6- } \\
\text { shagaol) } \\
\text { Dose: } 1 \mathrm{~g} \\
\text { (0.25g QID) } \\
\text { Duration: For } \\
\text { 6 days from } 3 \\
\text { days before } \\
\text { CTx session }\end{array}$ & $\begin{array}{l}\mathrm{n}: 40 \\
\text { Type: } \\
\text { Placebo } \\
\text { capsule } \\
\text { Dose: } \\
0.25 \mathrm{~g} \text { QID } \\
\text { made with } \\
\text { starch } \\
\text { Duration: For } \\
6 \text { days from } 3 \\
\text { days before } \\
\text { CTx session }\end{array}$ & 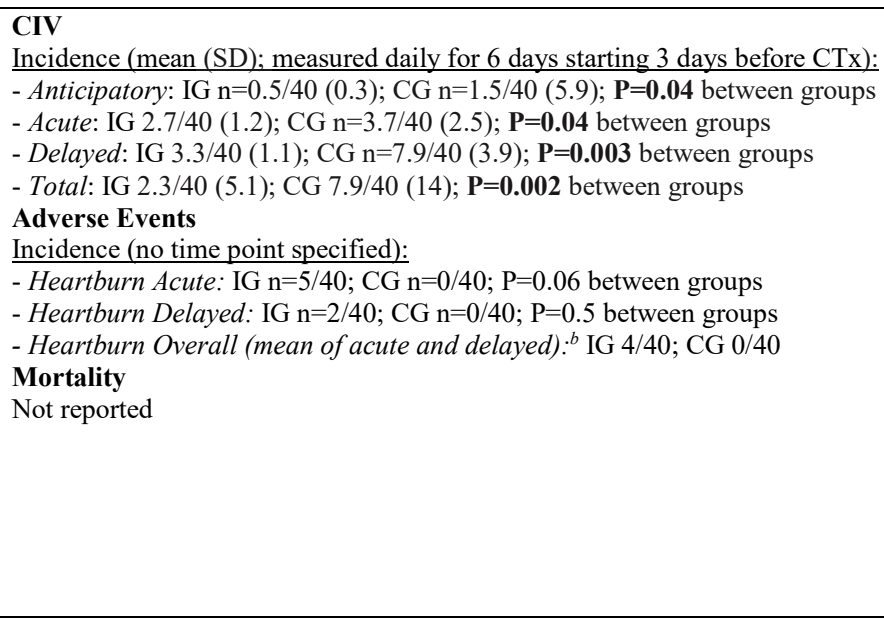 \\
\hline
\end{tabular}

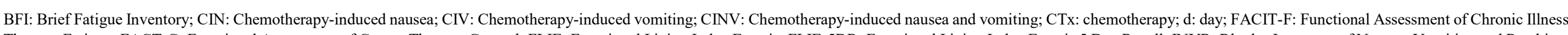

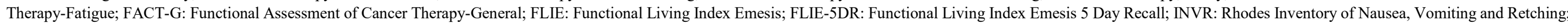

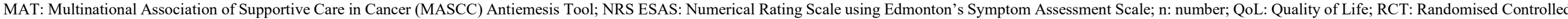
Trial; VAS: Visual analogue scale.

${ }^{a}$ Data tables not published, therefore data unclear.

${ }^{\mathrm{b}}$ This was calculated by review authors to generate a number used for meta analyses.

${ }^{\mathrm{c}}$ Information supplied by author. 
Table 2. Included studies located in the researchers' previous systematic review ( $\mathrm{n}=5$ ), examining the effect of ginger supplementation on chemotherapyinduced nausea and vomiting incidence and chemotherapy-related outcomes.

\begin{tabular}{|c|c|c|c|c|c|c|c|}
\hline Citation & $\begin{array}{l}\text { Study Design } \\
\text { \& Setting }\end{array}$ & Population & CTx Protocol & $\begin{array}{l}\text { Adjuvant } \\
\text { Therapies }\end{array}$ & $\begin{array}{l}\text { Intervention } \\
\text { Group/s (IG) }\end{array}$ & $\begin{array}{l}\text { Comparator } \\
\text { Group/s } \\
\text { (CG) }\end{array}$ & Results \\
\hline $\begin{array}{l}\text { Fahimi } \\
2011 \\
(28)\end{array}$ & $\begin{array}{l}\text { Design: } \\
\begin{array}{l}\text { Double blind } \\
\text { crossover } \\
\text { placebo RCT }\end{array} \\
\text { Country: Iran } \\
\text { Dates: Not } \\
\text { specified }\end{array}$ & 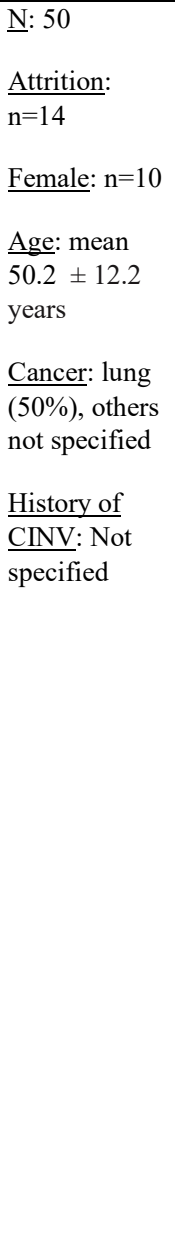 & $\begin{array}{l}\text { Drugs: } \\
\text { Cisplatin with } \\
\text { at least one of: } \\
\text { etoposide, } \\
\text { docetaxel, } \\
\text { gemcitabine, } \\
\text { docetaxel, } \\
\text { vinorelbine, } \\
\text { cyclophospham } \\
\text { ide, } \\
\text { paclitaxel, } \\
\text { doxorubicin, 5- } \\
\text { FU, } \\
\text { pemetrexed } \\
\frac{\text { Emetogenicity: }}{\text { Not specified. }} \\
\underline{\text { Single or multi }} \\
\text { day: Unclear } \\
\frac{\text { Commencing }}{\text { Cycle: Not }} \\
\text { specified } \\
\frac{\text { Cycle length: }}{\text { Not specified }}\end{array}$ & $\begin{array}{l}\text { 5-HT3 } \\
\text { antagonist } \\
\text { (granisetrone) } \\
\text { and } \\
\text { corticostreoid } \\
\text { (hydrocortiso } \\
\text { ne) }\end{array}$ & $\begin{array}{l}\text { n: } 36 \\
\text { Type: } 0.25 \mathrm{~g} \\
\text { capsule, powdered } \\
\text { ginger (Zintoma) } \\
\text { Dose: } 1 \mathrm{~g}(2 \mathrm{x} \\
0.25 \mathrm{~g} \mathrm{BD}) \\
\text { Duration: } 3 \text { days } \\
\text { starting on the day } \\
\text { of CTx then a 3- } \\
\text { week washout } \\
\text { period prior to } \\
\text { cross over }\end{array}$ & $\begin{array}{l}\text { n: } 36 \\
\text { Type: } 0.25 \mathrm{~g} \\
\text { placebo } \\
\text { capsule } \\
\text { composed of } \\
\text { lactose. } \\
\text { Dose: } 1 \mathrm{~g}(2 \mathrm{x} \\
0.25 \mathrm{~g} B \mathrm{BD} \\
\text { Duration: } 3 \\
\text { days starting } \\
\text { on the day of } \\
\text { CTx then a 3- } \\
\text { week washout } \\
\text { period prior } \\
\text { to cross over }\end{array}$ & 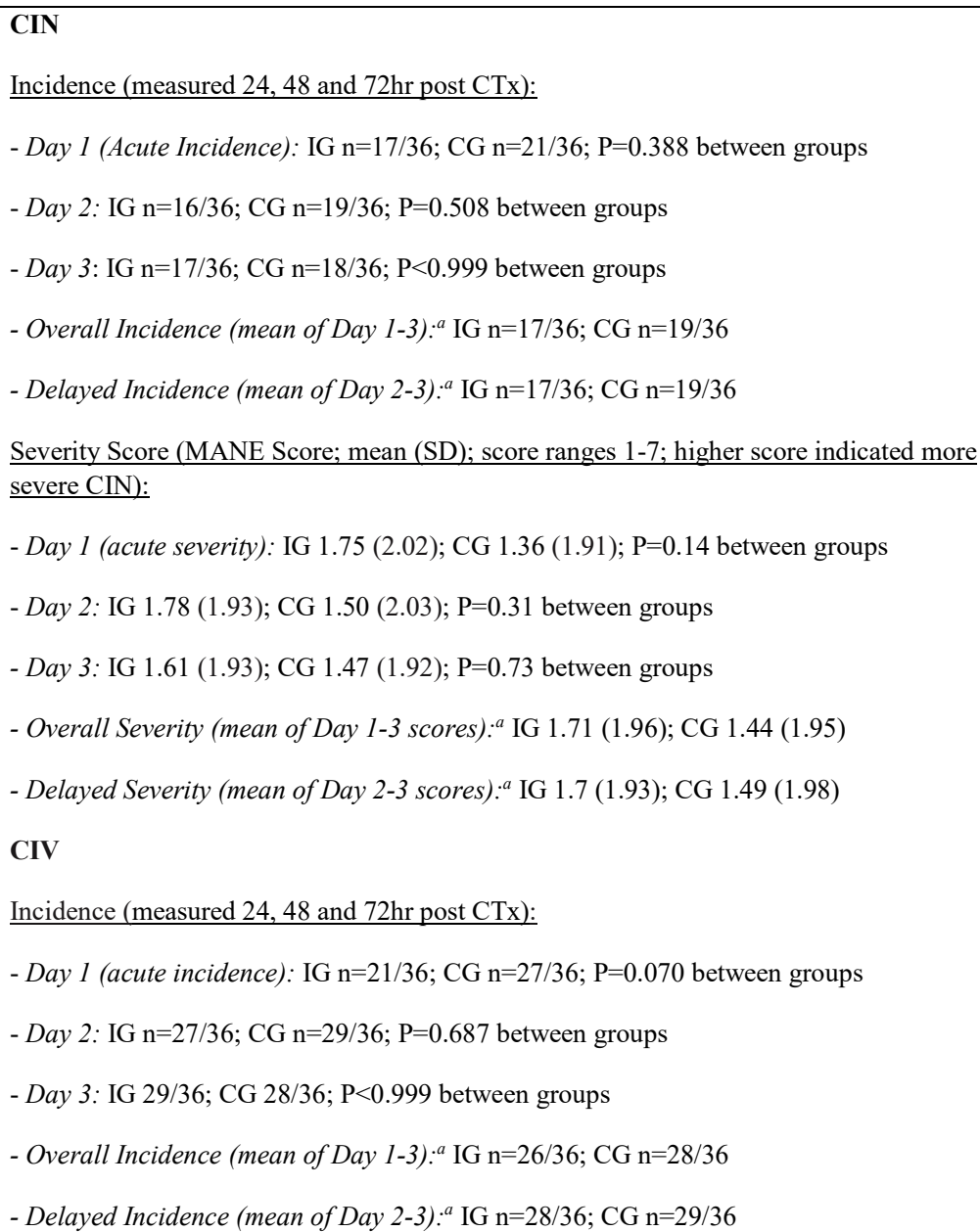 \\
\hline
\end{tabular}




\begin{tabular}{|c|c|c|c|c|c|c|c|}
\hline & & & & & & & $\begin{array}{l}\text { Severity Score (MANE Score; mean (SD); score ranges 1-7; higher score indicated more } \\
\text { severe CIV): } \\
\text { - Day 1: IG } 1.47 \text { (2.18); CG } 0.94 \text { (1.77); } \mathrm{P}=0.14 \text { between groups } \\
\text { - Day 2: IG } 1.03 \text { (1.89); CG } 0.83 \text { (1.84); } \mathrm{P}=0.72 \text { between groups } \\
\text { - Day 3: IG } 0.80 \text { (1.83); CG } 0.92 \text { (1.86); } \mathrm{P}=0.78 \text { between groups } \\
\text { Mortality } \\
\text { Not reported }\end{array}$ \\
\hline $\begin{array}{l}\text { Manusi- } \\
\text { rivithaya } \\
2004 \\
(34)\end{array}$ & $\begin{array}{l}\text { Design: } \\
\text { Double blind } \\
\text { crossover } \\
\text { RCT } \\
\text { Country: } \\
\text { Thailand } \\
\text { Dates: Not } \\
\text { specified }\end{array}$ & $\begin{array}{l}\text { N: } 48 \\
\text { Attrition: } n=5 \\
\text { Female: } n=43 \\
\text { Age: mean } \\
46 \pm 10-14 \\
\text { years } \\
\text { Cancer: ovary } \\
(76 \%), \\
\text { cervical } \\
(23 \%) \\
\text { History of } \\
\text { CINV: Not } \\
\text { specified }\end{array}$ & 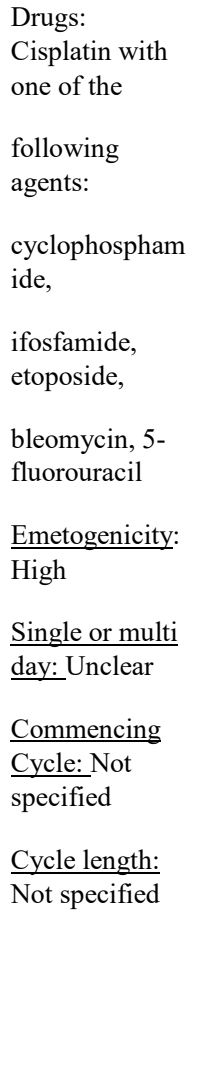 & $\begin{array}{l}\text { Metocloprami } \\
\text { de (Day } 130 \\
\text { min before } \\
\text { CTx), } \\
\text { dexamethason } \\
\text { e and } \\
\text { lorazepam } \\
\text { (Day } 130 \\
\text { min before } \\
\text { and } 6 \text { h after } \\
\text { CTx) }\end{array}$ & $\begin{array}{l}\text { n: } 43 \\
\text { Type: } 0.25 \mathrm{~g} \\
\text { capsule, ginger } \\
\text { root powder } \\
\text { Dose: } 1 \mathrm{~g}(0.25 \mathrm{~g} \\
\text { QID) } \\
\text { Duration: } 5 \text { days } \\
\text { then a 3-4 week } \\
\text { washout period } \\
\text { prior to cross over }\end{array}$ & $\begin{array}{l}\text { n: } 43 \\
\text { Type: } 0.25 \mathrm{~g} \\
\text { placebo } \\
\text { capsule (corn } \\
\text { starch) } \\
\text { Dose: } 1 \mathrm{~g} \\
\text { (0.25g QID) } \\
\text { Duration: } 5 \\
\text { days then a 3- } \\
4 \text { week } \\
\text { washout } \\
\text { period prior } \\
\text { to cross over }\end{array}$ & 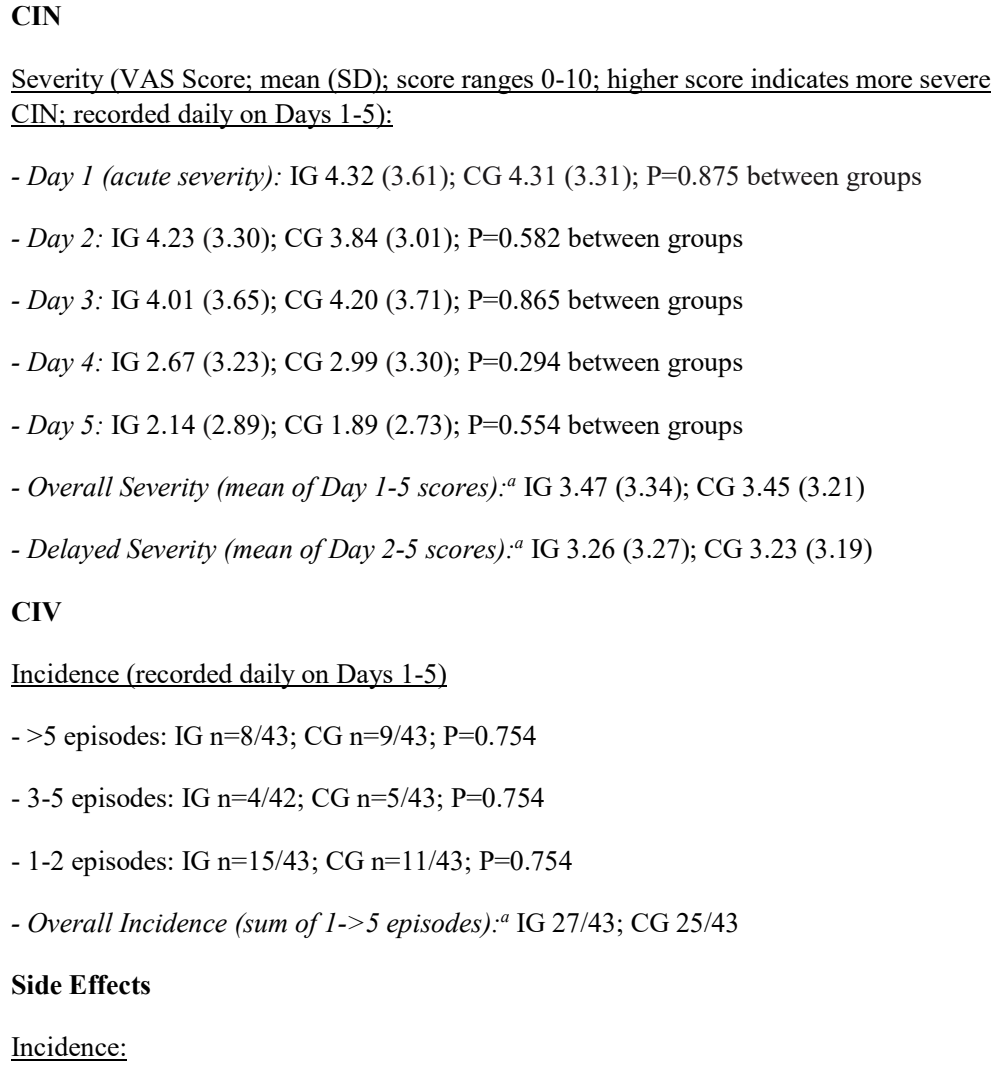 \\
\hline
\end{tabular}




\begin{tabular}{|c|c|c|c|c|c|c|c|}
\hline & & & & & & & $\begin{array}{l}\text { - Any relatable side effects: } \mathrm{IG} \mathrm{n}=12 / 43 ; \mathrm{CG} \mathrm{n}=8 / 43 ; \mathrm{P}=0.503 \text { between groups } \\
\text { - Restlessness: } \mathrm{IG} \mathrm{n}=8 / 46 ; \mathrm{n}=\mathrm{CG} 2 / 46 ; \mathrm{P}=0.109 \text { between groups } \\
\text { - Diarrhoea: } \mathrm{IG} \mathrm{n}=2 / 46 ; \mathrm{CG} \mathrm{n}=6 / 46 ; \mathrm{P}=0.289 \text { between groups } \\
\text { - Constipation: } \mathrm{IG} \mathrm{n}=6 / 46 ; \mathrm{CG} \mathrm{n}=3 / 46 ; \mathrm{P}=0.508 \text { between groups } \\
\text { - Headache: } \mathrm{IG} \mathrm{n}=3 / 46 ; \mathrm{CG} \mathrm{n}=1 / 46 ; \mathrm{P}=0.625 \text { between groups } \\
\text { - Dizziness: } \mathrm{IG} \mathrm{n}=5 / 46 ; \mathrm{CG} \mathrm{n}=6 / 46 ; \mathrm{P}=1.000 \text { between groups } \\
\text { - Heartburn: } \mathrm{IG} \mathrm{n}=3 / 46 ; \mathrm{CG} \mathrm{n}=3 / 46 ; \mathrm{P}=1.000 \text { between groups } \\
\text { - Palpitation: } \mathrm{IG} \mathrm{n}=1 / 46 ; \mathrm{CG} \mathrm{n}=1 / 46 ; \mathrm{P}=1.000 \text { between groups } \\
\text { - Akathisia: } \mathrm{IG} \mathrm{n}=1 / 46 ; \mathrm{CG} \mathrm{n}=0 / 46 \\
\text { - Acute Dystonic Reaction: } \mathrm{CG} \mathrm{n}=1 / 46 ; \mathrm{CG} \mathrm{n}=0 / 46 \\
\text { Mortality } \\
\text { Not reported }\end{array}$ \\
\hline $\begin{array}{l}\text { Panahi } \\
2012 \\
(29)\end{array}$ & $\begin{array}{l}\text { Design: Open } \\
\text { pilot RCT } \\
\text { Country: Iran } \\
\text { Study } \\
\text { Duration } \\
\text { Dates: July } \\
2008 \text { to } \\
\text { November } \\
2009\end{array}$ & 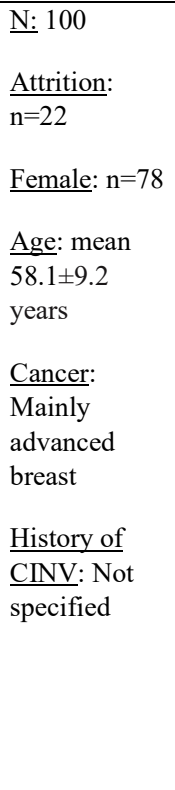 & $\begin{array}{l}\text { Drugs: } \\
\text { Predominately } \\
\text { TEC } \\
\text { (docetaxel, } \\
\text { epirubicin, } \\
\text { cyclophospham } \\
\text { ide) } \\
\text { Emetogenicity: } \\
\text { Moderate or } \\
\text { High } \\
\text { Single or multi } \\
\text { day: Not } \\
\text { specified } \\
\text { Commencing } \\
\text { Cycle: Mainly } \\
\text { Cycle 1 }\end{array}$ & $\begin{array}{l}\text { Standard } \\
\text { antiemetic } \\
\text { regimen } \\
\text { (granisetron } \\
\text { and } \\
\text { dexamethason } \\
\text { e) }\end{array}$ & $\begin{array}{l}\underline{\mathrm{n}}: 37 \\
\text { Type: } 0.5 \mathrm{~g} \\
\text { capsule powdered } \\
\text { ginger root } \\
\text { Dose: } 1.5 \mathrm{~g}(0.5 \mathrm{~g} \\
\text { TID) } \\
\text { Duration: } 5 \text { days } \\
\text { from the day of } \\
\text { CTx. Number of } \\
\text { Cycles } \\
\text { unspecified }\end{array}$ & $\begin{array}{l}\underline{\mathrm{n}:} 41 \\
\frac{\text { Type: Usual }}{\text { care }}\end{array}$ & 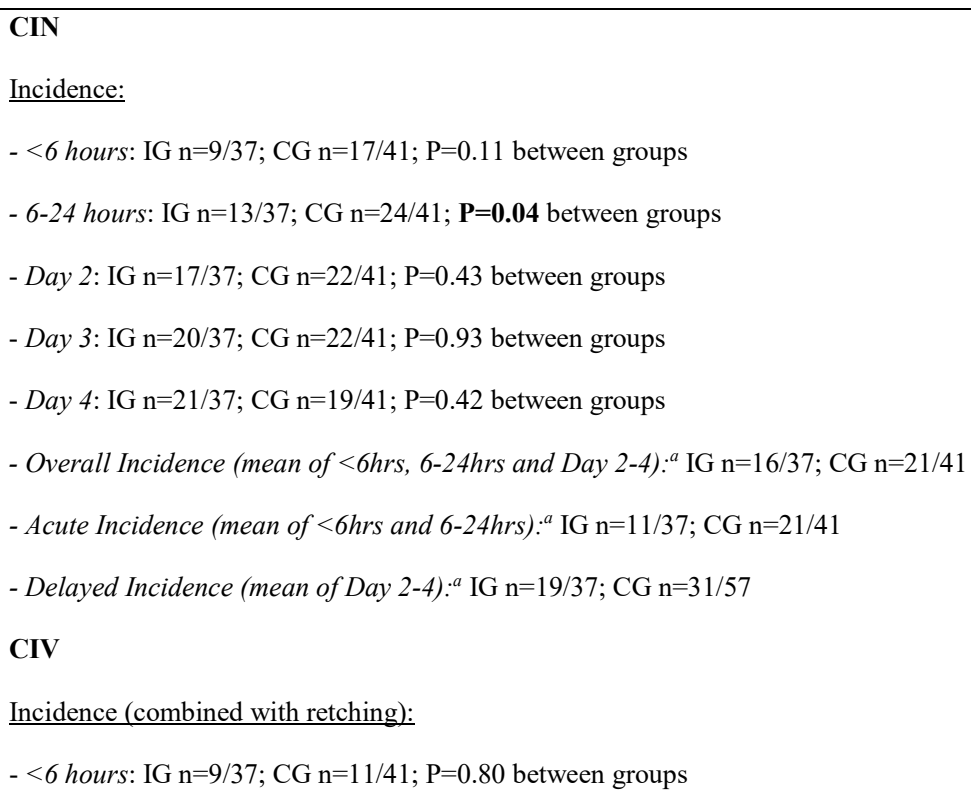 \\
\hline
\end{tabular}




\begin{tabular}{|c|c|c|c|c|c|c|c|}
\hline & & & $\begin{array}{l}\text { Cycle length: } \\
\text { Not specified }\end{array}$ & & & & 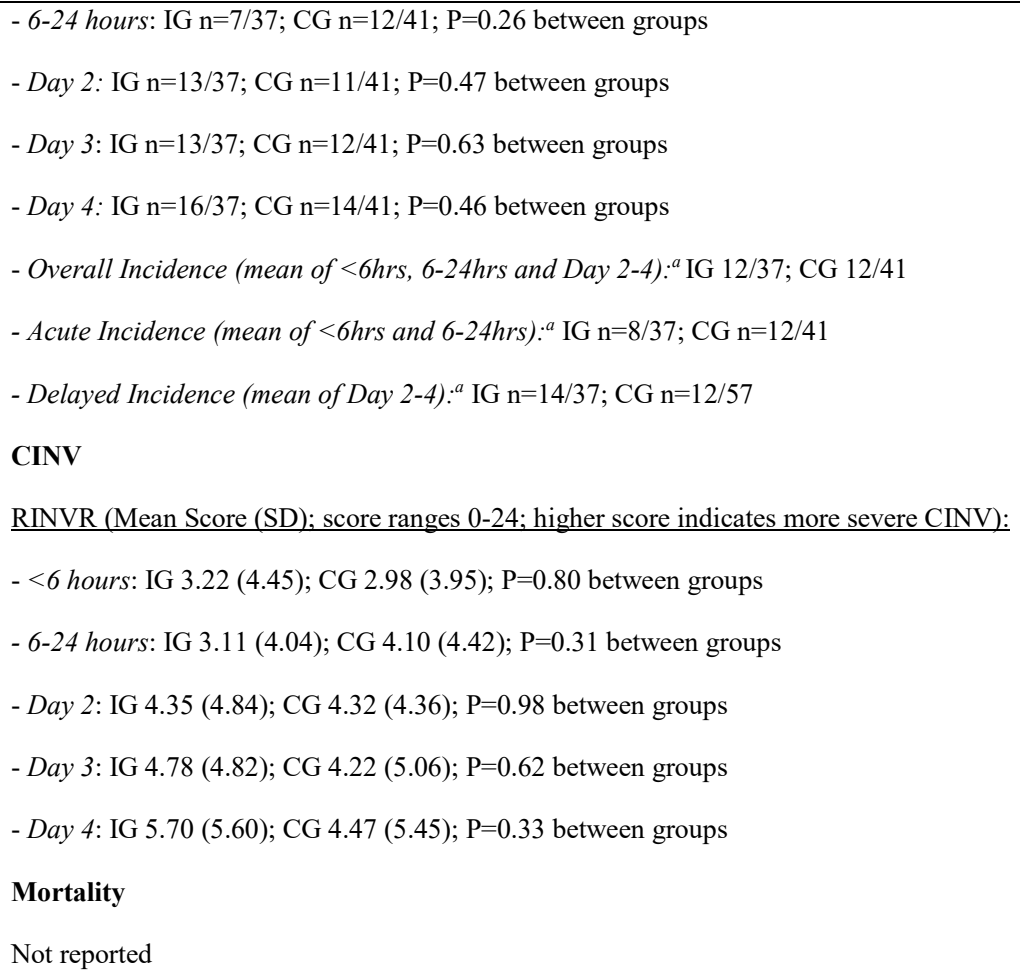 \\
\hline $\begin{array}{l}\text { Ryan } \\
2012 \\
(27)\end{array}$ & $\begin{array}{l}\text { Design: } \\
\begin{array}{l}\text { Double blind, } \\
\text { placebo RCT }\end{array} \\
\text { Country: USA } \\
\text { Recruitment } \\
\text { Dates: June } \\
\text { 2002 to } \\
\text { December } \\
2008\end{array}$ & 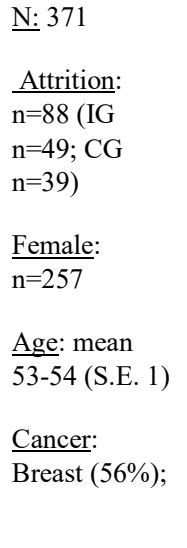 & $\begin{array}{l}\text { Drugs: Not } \\
\text { specified } \\
\text { Emetogenicity: } \\
\text { Any } \\
\frac{\text { Single or multi }}{\text { day: Not }} \\
\text { specified } \\
\frac{\text { Commencing }}{\text { Cycle: } \geq \text { Cycle }} \\
2 \\
\text { Cycle length: } \\
\text { Not specified }\end{array}$ & $\begin{array}{l}\text { 5-HT3 } \\
\text { receptor } \\
\text { antagonist. } \\
\text { dexamathason } \\
\text { e at all CTx } \\
\text { Cycles }\end{array}$ & $\begin{array}{l}\text { n: } 134 \\
\text { Type 1: } 0.25 \mathrm{~g} \\
\text { capsule ginger } \\
\text { liquid extract of } \\
\text { ginger root ( } 8.5 \mathrm{mg} \\
\text { of combined } \\
\text { gingerols, } \\
\text { zingerone and } \\
\text { shogoal) with } \\
\text { olive oil } \\
\text { containing other } \\
\text { unspecified } \\
\text { excipients to } \\
\text { improve } \\
\text { solubilisation and }\end{array}$ & $\begin{array}{l}\text { n: } 149 \\
\text { Type: } 0.25 \mathrm{~g} \\
\text { placebo } \\
\text { capsule with } \\
\text { olive oil and } \\
\text { excipients } \\
\text { Dose: } 1.5 \mathrm{~g} \\
\text { (3x0.25g BD) } \\
\text { Duration: } 6 \\
\text { days, starting } \\
\text { three days } \\
\text { before CTx. }\end{array}$ & 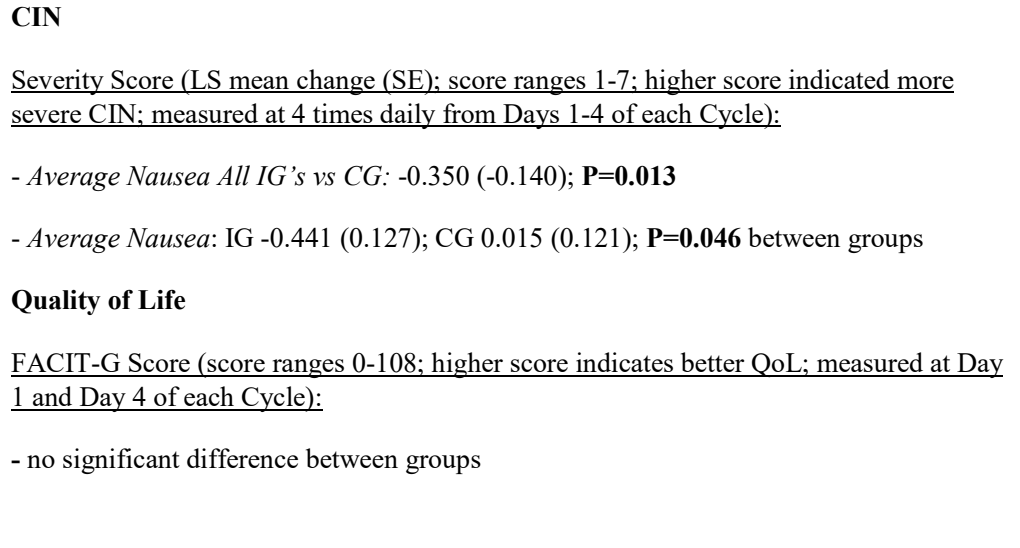 \\
\hline
\end{tabular}




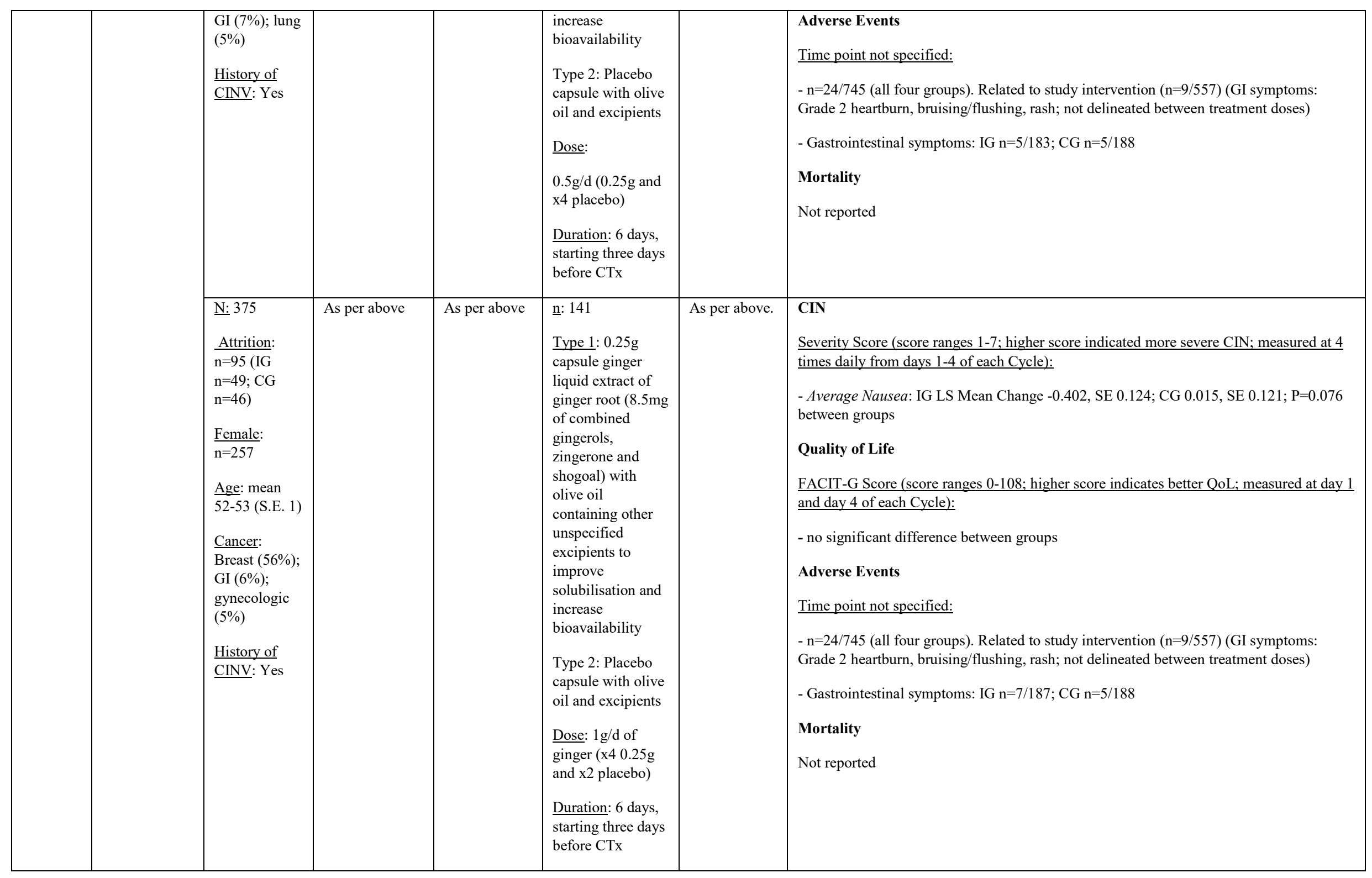




\begin{tabular}{|c|c|c|c|c|c|c|c|}
\hline & & 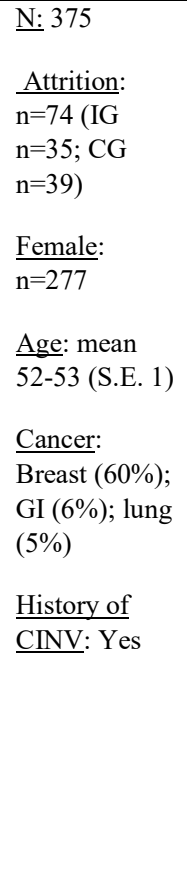 & As per above & As per above & $\begin{array}{l}\text { n: } 152 \\
\text { Type 1: } 0.25 \mathrm{~g} \\
\text { capsule ginger } \\
\text { liquid extract of } \\
\text { ginger root ( } 8.5 \mathrm{mg} \\
\text { of combined } \\
\text { gingerols, } \\
\text { zingerone and } \\
\text { shogoal) with } \\
\text { olive oil } \\
\text { containing other } \\
\text { unspecified } \\
\text { excipients to } \\
\text { improve } \\
\text { solubilisation and } \\
\text { increase } \\
\text { bioavailability } \\
\text { Dose: } 1.5 \mathrm{~g} / \mathrm{d} \text { (x6 } \\
0.25 \mathrm{~g} ; \text { no } \\
\text { placebo). } \\
\text { Duration: } 6 \text { days, } \\
\text { starting three days } \\
\text { before CTx }\end{array}$ & As per above. & $\begin{array}{l}\text { CIN } \\
\text { Severity Score (score ranges 1-7; higher score indicated more severe CIN; measured at } 4 \\
\text { times daily from Days 1-4 of each Cycle): } \\
\text { - Average Nausea: IG C LS Mean Change -0.158, SE 0.120; CG 0.015, SE 0.121; } \\
\mathrm{P}=0.738 \text { between groups } \\
\text { Quality of Life } \\
\text { FACIT-G Score (score ranges } 0-108 \text {; higher score indicates better QoL; measured at day } 1 \\
\text { and day } 4 \text { of each Cycle): } \\
\text { - no significant difference between groups } \\
\text { Adverse Events } \\
\text { Time point not specified: } \\
\text { - n= } 24 / 745 \text { (all four groups). Related to study intervention ( } \mathrm{n}=9 / 557) \text { (GI symptoms: } \\
\text { Grade } 2 \text { heartburn, bruising/flushing, rash; not delineated between treatment doses) } \\
\text { - Gastrointestinal symptoms: IG } \mathrm{n}=9 / 187 ; \text { CG n=5/188 } \\
\text { Mortality } \\
\text { Not reported }\end{array}$ \\
\hline $\begin{array}{l}\text { Zick } \\
2009 \\
(38)\end{array}$ & $\begin{array}{l}\text { Design: } \\
\text { Double blind } \\
\text { Placebo RCT } \\
\text { Country: USA } \\
\underline{\text { Study }} \\
\text { Duration } \\
\frac{\text { Dates: June }}{2003 \text { and May }} \\
2006\end{array}$ & $\begin{array}{l}\underline{\mathrm{N}}: 110 \\
\text { Attrition: } \\
\mathrm{n}=21(\mathrm{IG} \\
\mathrm{n}=10 ; \mathrm{GC} \\
\mathrm{n}=11) \\
\text { Female: } \mathrm{n}=92 \\
\text { Age: mean } \\
\text { 53-55 } \pm 11-12 \\
\text { years } \\
\text { Cancer: Not } \\
\text { specified }\end{array}$ & $\begin{array}{l}\text { Drugs: } 20 \\
\text { different } \\
\text { regimens } \\
\text { specified in } \\
\text { publication } \\
\text { Emetogenicity: } \\
17 \% \text { high; } 65 \% \\
\text { moderate. } \\
\text { Single or multi } \\
\text { day: Single } \\
\text { Commencing } \\
\text { Cycle: } \geq \text { Cycle } \\
2\end{array}$ & $\begin{array}{l}\text { Apripetant, 5- } \\
\text { HT3 receptor } \\
\text { antagonist } \\
\text { (Dolasetron, } \\
\text { Granistron, } \\
\text { Ondansetron } \\
\text { or } \\
\text { Palonosetron) }\end{array}$ & $\begin{array}{l}\text { n: } 53 \\
\text { Type: } 0.25 \mathrm{~g} \\
\text { capsule dry } \\
\text { extract of ginger } \\
\text { root }(10: 1(\mathrm{v} / \mathrm{v}) \\
\text { extraction solvent } \\
\text { (ethanol } \\
50 \%) / \text { root) } \\
\text { standardized to } 15 \\
\mathrm{mg}(5 \%) \text { of total } \\
\text { gingerols. } 5.38 \mathrm{mg} \\
(2.15 \%) 6- \\
\text { gingerol, } 1.80 \mathrm{mg} \\
(0.72 \%) 8- \\
\text { gingerol, } 4.19 \mathrm{mg} \\
(1.78 \%) 10- \\
\text { gingerol, and } 0.92\end{array}$ & $\begin{array}{l}\text { n: } 57 \\
\text { Type: } \\
\text { Placebo } \\
\text { capsules with } \\
\text { lactose } \\
\text { powder } \\
\text { Dose: } 8 / \text { day } \\
\text { Duration: } 3 \\
\text { days starting } \\
\text { on day of } \\
\text { CTx } \\
\text { administratio } \\
\mathrm{n}\end{array}$ & $\begin{array}{l}\text { CIN } \\
\text { Incidence: } \\
\text { - <24hr post } C T x \text { (acute incidence): IG } \mathrm{n}=33 / 53 ; \mathrm{CG} \mathrm{n}=31 / 57 ; \mathrm{P}=0.86 \text { between groups } \\
\text { - 24-48hr post } C T x \text { (delayed incidence): } \mathrm{IG} \mathrm{n}=37 / 53 ; \mathrm{CG} \mathrm{n}=31 / 57 ; \mathrm{P}=0.16 \text { between } \\
\text { groups } \\
\text { - Overall (mean of }<24 h r s \text { and } 24-48 h r s):^{a} \mathrm{IG} \mathrm{n}=35 / 53 ; \mathrm{CG} \mathrm{n}=31 / 57 \\
\text { Severity (MANE Score; mean (SD); score ranges } 1-7 ; \text { higher score indicated more severe } \\
\text { CIN): } \\
-<24 h r \text { post } C T x \text {, no aprepitant: } \mathrm{IG} 3.1(1.2) \mathrm{CG} 2.8(1.3) ; \mathrm{P}=0.47 \\
-<24 h r \text { post } C T x \text {, aprepitant: } \mathrm{IG} 2.8(1.1) ; \mathrm{CG} 3.1(1.5) ; \mathrm{P}=0.55\end{array}$ \\
\hline
\end{tabular}




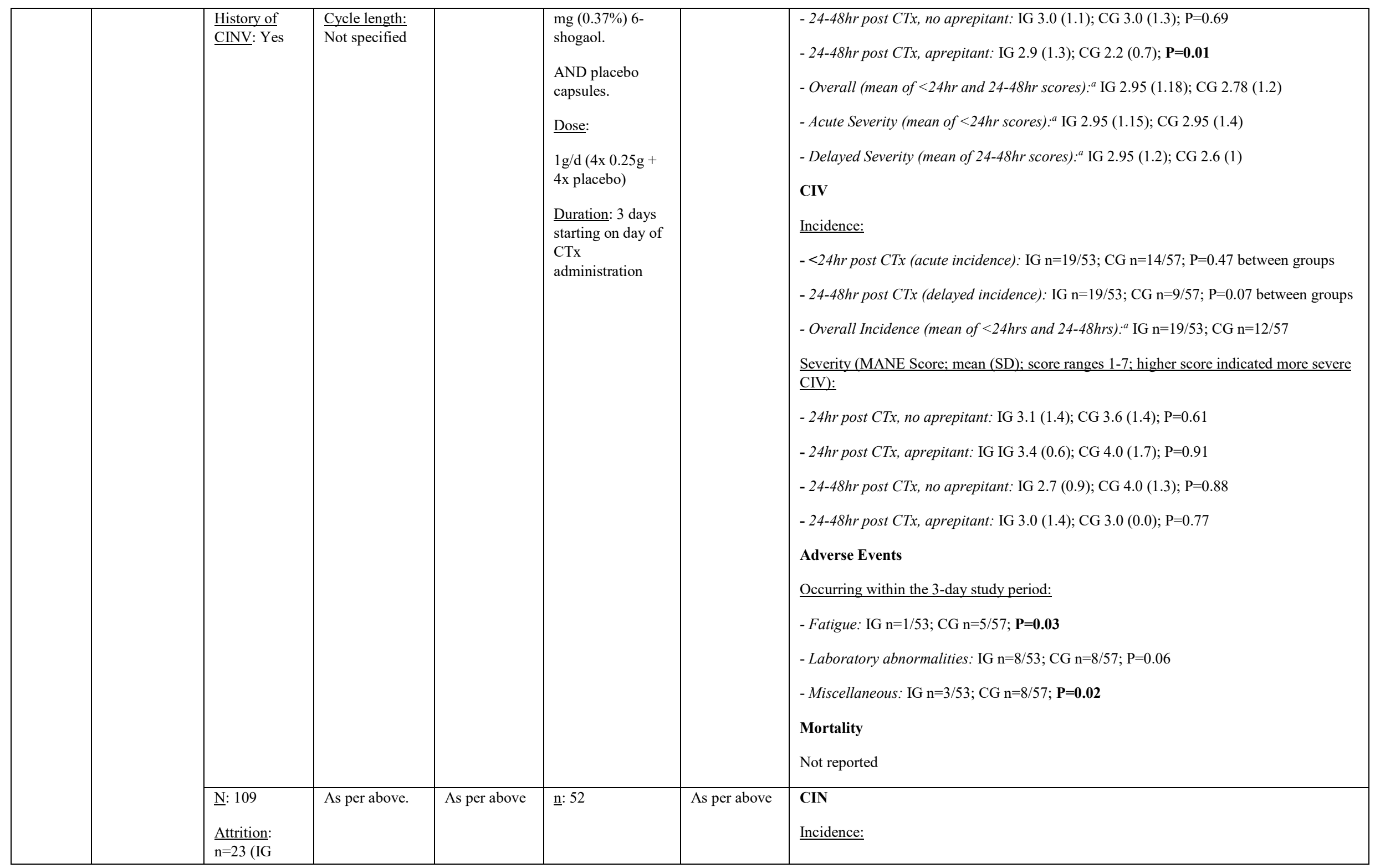




\begin{tabular}{|c|c|c|c|c|c|}
\hline & & $\begin{array}{l}\mathrm{n}=12 ; \mathrm{GC} \\
\mathrm{n}=11) \\
\text { Female: } \mathrm{n}=83 \\
\text { Age: mean } \\
55-58 \pm 11-12 \\
\text { years } \\
\text { Cancer: Not } \\
\text { specified } \\
\text { History of } \\
\text { CINV: Yes }\end{array}$ & $\begin{array}{l}\text { Emetogenicity: } \\
19 \% \text { high; } 63 \% \\
\text { moderate }\end{array}$ & $\begin{array}{l}\text { Type: As per } \\
\text { above, no placebo. } \\
\text { Dose: } 2 \mathrm{~g} / \mathrm{d}(8 \mathrm{x} \\
0.25 \mathrm{~g}) \\
\text { Duration: } 3 \text { days } \\
\text { starting on day of } \\
\text { CTx } \\
\text { administration }\end{array}$ & 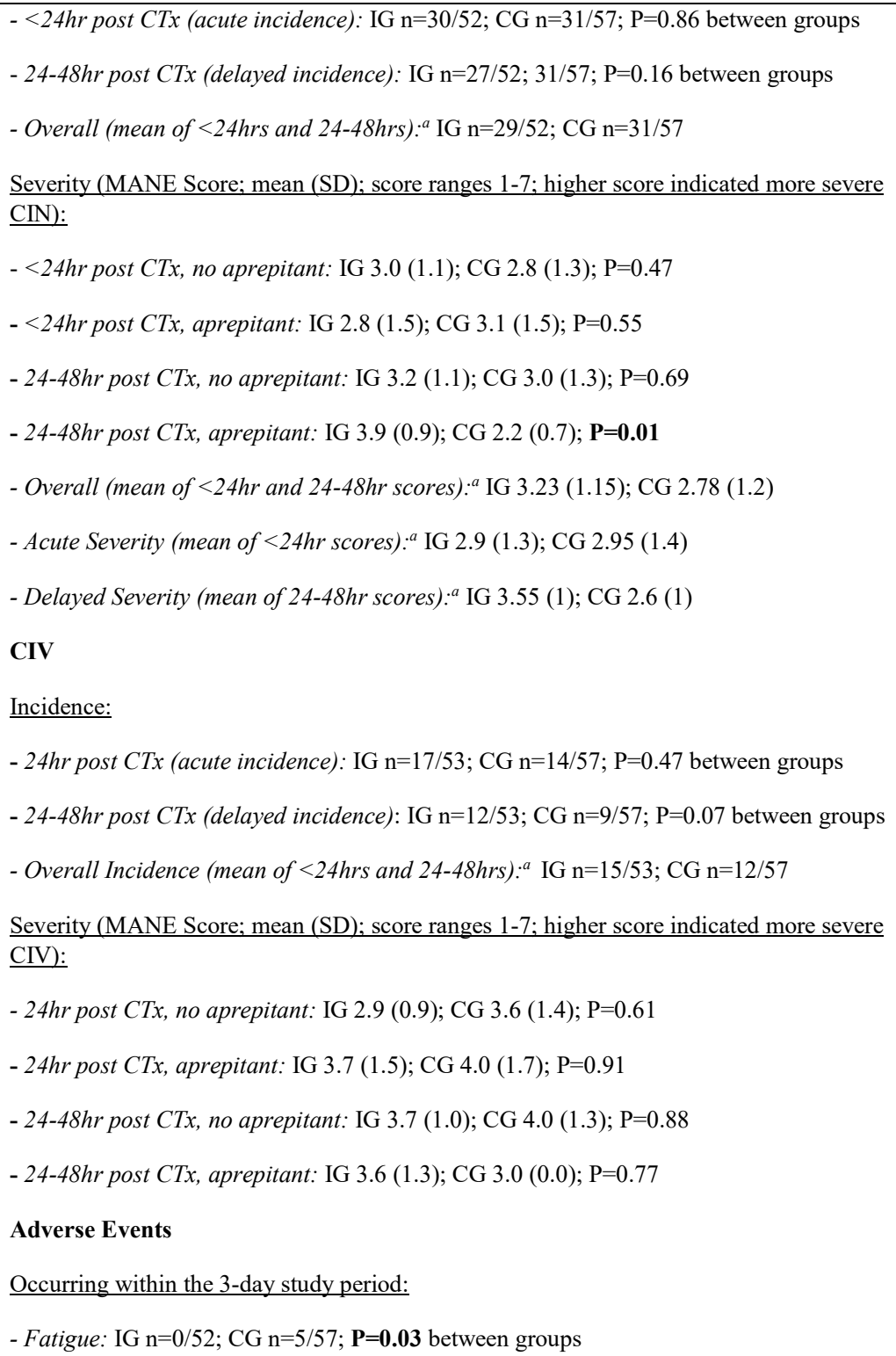 \\
\hline
\end{tabular}




\begin{tabular}{|l|l|l|l|l|l|l|}
\hline & & & & & & - Laboratory abnormalities: IG $\mathrm{n}=1 / 52 ;$ CG $\mathrm{n}=8 / 57 ; \mathrm{P}=0.06$ between groups \\
- Miscellaneous: IG $\mathrm{n}=1 / 52 ;$ CG $\mathrm{n}=8 / 57 ; \mathbf{P}=\mathbf{0 . 0 2}$ between groups \\
Mortality \\
\end{tabular}

CIN: chemotherapy induced nausea; CIV: chemotherapy induced vomiting; CINV: chemotherapy induced nausea and vomiting; CTx: chemotherapy; ESAS: Edmonton's Symptom Assessment Scale; FACT-G: Functional Assessment of Cancer Therapy-General; GI: gastrointestinal; LS Mean: least squares mean; MANE: Morrow Assessment of Nausea and Emesis; N: number; RCT: Randomised Controlled Trial; RINVR: Rhodes Index of Nausea, Vomiting, and Retching; VAS: Visual Analogue Score

${ }^{a}$ This was calculated by review authors to generate a number used for meta analyses. 
Online Supplementary Material 4: Non-significant meta-analyses forest plots examining the efficacy and safety of ginger for ameliorating chemotherapy-induced nausea and vomiting

\begin{tabular}{|c|c|c|c|c|c|c|c|c|c|}
\hline Study or Subgroup & \multicolumn{2}{|c|}{ Ginger } & \multicolumn{2}{|c|}{ Comparator } & Weight & $\begin{array}{c}\text { Odds Ratio } \\
\text { M-H, Random, } 95 \% \mathrm{CI}\end{array}$ & \multicolumn{3}{|c|}{$\begin{array}{c}\text { Odds Ratio } \\
\mathrm{M}-\mathrm{H} \text {, Random, } 95 \% \mathrm{Cl}\end{array}$} \\
\hline Alparslan 2012 & 0 & 15 & 23 & 30 & $1.8 \%$ & $0.01[0.00,0.19]$ & $\longleftarrow$ & & \\
\hline Bossi 2017 & 65 & 121 & 63 & 123 & $18.2 \%$ & $1.11[0.67,1.83]$ & & & \\
\hline Fahimi 2011 & 17 & 36 & 19 & 36 & $11.0 \%$ & $0.80[0.32,2.02]$ & & & \\
\hline Konmun 2017 & 30 & 40 & 37 & 41 & $7.5 \%$ & $0.32[0.09,1.14]$ & & & \\
\hline Li 2018 & 46 & 71 & 45 & 69 & $14.6 \%$ & $0.98[0.49,1.97]$ & & & \\
\hline Panahi 2012 & 16 & 37 & 21 & 41 & $11.5 \%$ & $0.73[0.30,1.77]$ & & & \\
\hline Shokri 2017 & 8 & 20 & 14 & 29 & $8.4 \%$ & $0.71[0.23,2.26]$ & & & \\
\hline Zick 2009 & 35 & 53 & 31 & 57 & $13.3 \%$ & $1.63[0.75,3.53]$ & & & \\
\hline Zick 2009b & 29 & 52 & 31 & 57 & $13.6 \%$ & $1.06[0.50,2.25]$ & & & \\
\hline Total $(95 \% \mathrm{CI})$ & & 445 & & 483 & $100.0 \%$ & $0.84[0.56,1.27]$ & & & \\
\hline Total events & 246 & & 284 & & & & & & \\
\hline $\begin{array}{l}\text { Heterogeneity. } \mathrm{Tau}^{2}= \\
\text { Test for overall effect }\end{array}$ & $\begin{array}{l}0.18 ; C h \\
z=0.81\end{array}$ & $\begin{array}{l}i^{2}=1 \\
(P=\end{array}$ & $\begin{array}{l}51, \mathrm{df}= \\
.42 \mathrm{~J}\end{array}$ & (P & 05); & $=48 \%$ & $\frac{1}{0.1}$ & $\begin{array}{l}1 \\
0.5\end{array}$ & $\begin{array}{ccc}\frac{1}{2} & 5 & 1 \\
\text { Favours Comparator } & 10\end{array}$ \\
\hline
\end{tabular}

Figure 1. Ginger supplementation of any dose or duration had no association with likelihood of overall nausea (OR: $0.84,95 \%$ CI: $0.56-1.27 ; \mathrm{P}=0.42 ; \mathrm{n}=8$ studies; $\mathrm{n}=9$ interventions; $\mathrm{n}=928$ participants; $\mathrm{I}^{2}=48 \%$ ).

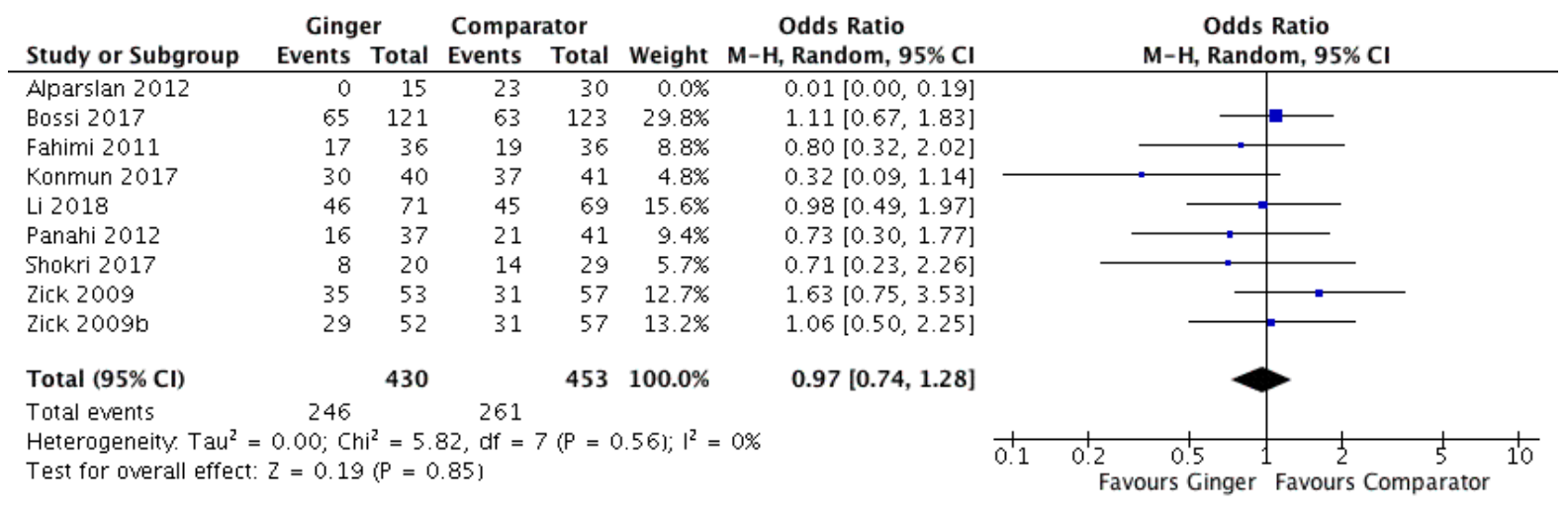

Figure 2. Ginger supplementation of any dose or duration had no association with likelihood of overall nausea (OR: $0.97,95 \% \mathrm{CI}$ : $0.74-1.28 ; \mathrm{P}=0.82 ; \mathrm{n}=7$ studies; $\mathrm{n}=8$ interventions; $\mathrm{n}=883$ participants; $\mathrm{I}^{2}=0 \%$; GRADE level: moderate). Sensitivity analysis: studies with high risk of bias ( $>70 \%$ of Cochrane Risk of Bias domains rated as unclear or high risk of bias) deselected; sensitivity analysis according to dose $(\leq />1 \mathrm{~g} /$ day) or duration $(\leq />3$ days) did not result in significant findings. 


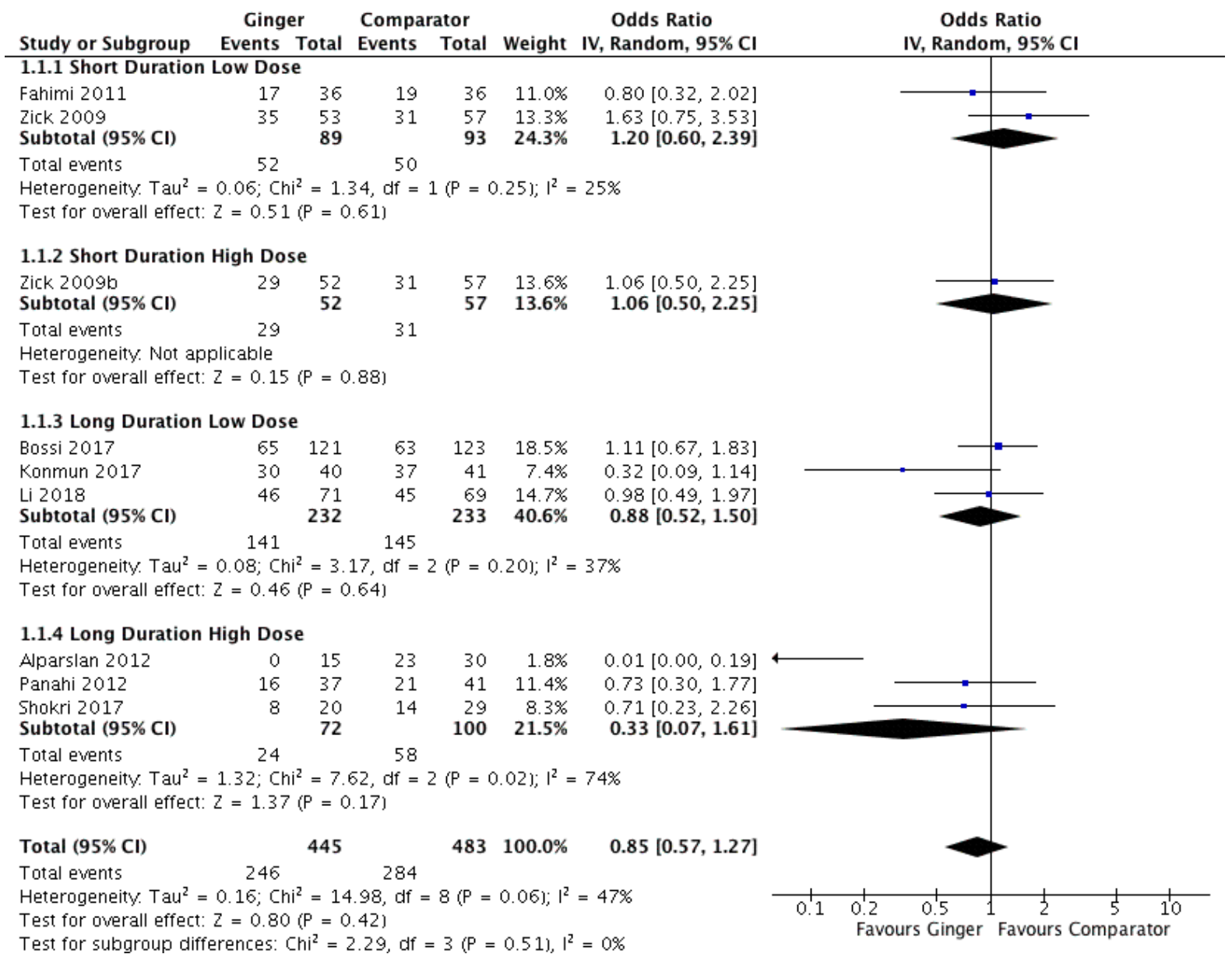

Figure 3. Ginger supplementation of any dose or duration had no association with likelihood of overall nausea with subgroup analysis using the four categories outlined in the meta-analysis method of varied duration and dosage (OR: $0.85,95 \%$ CI: $0.57-1.27 ; \mathrm{P}=0.51 ; \mathrm{n}=7$ studies; $\mathrm{n}=8$ interventions; $n=928$ participants; $\mathrm{I}^{2}=47 \%$ ). 


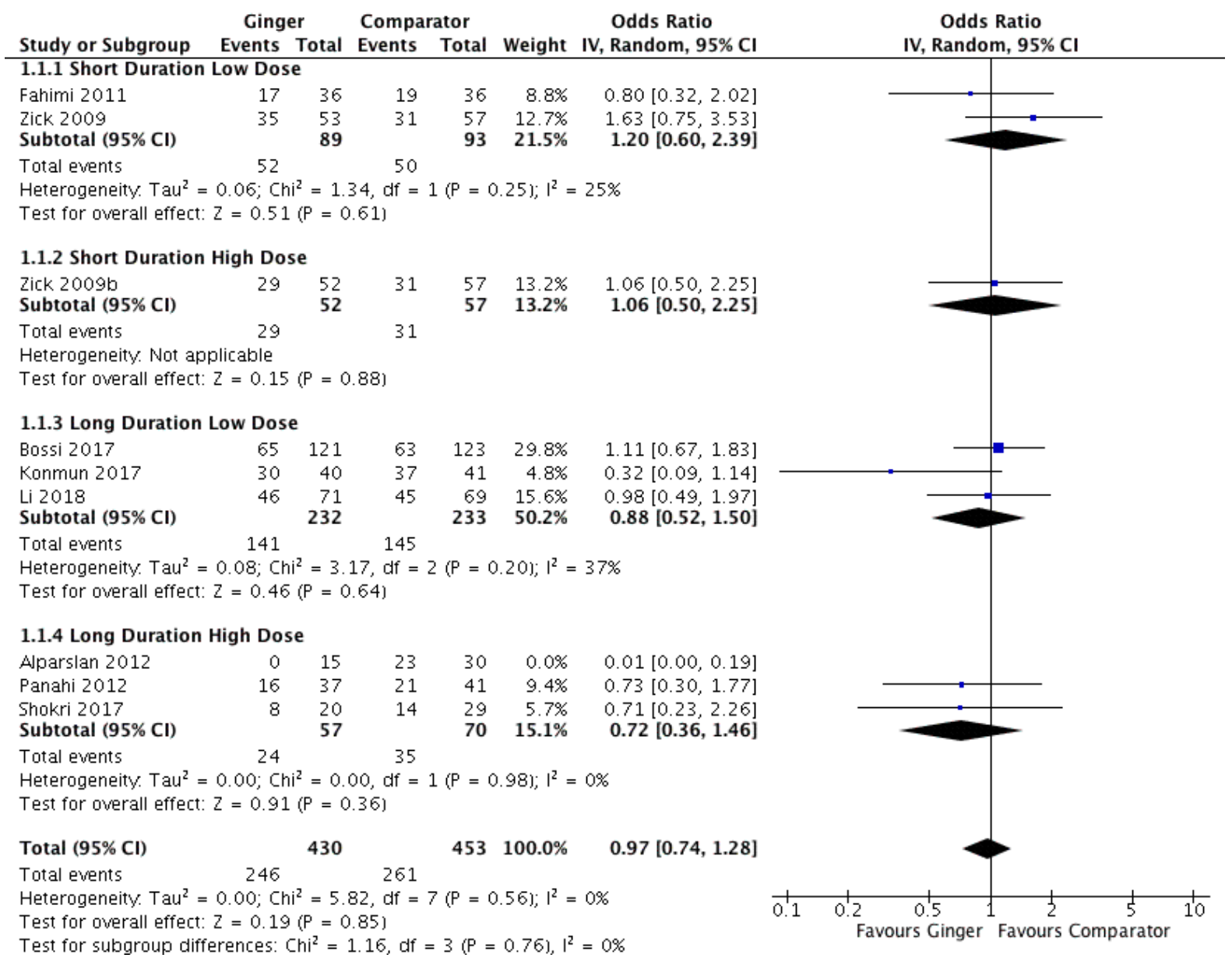

Figure 4. Ginger supplementation of any dose or duration had no association with likelihood of overall nausea with subgroup analysis using the four categories outlined in the meta-analysis method of varied duration and dosage (OR: 0.97, 95\% CI: 0.74-1.28; $\mathrm{P}=0.76 ; \mathrm{n}=7$ studies; $\mathrm{n}=8$ interventions; $\mathrm{n}=883$ participants; $\mathrm{I}^{2}=0 \%$ ). Sensitivity analysis: studies with high risk of bias ( $>70 \%$ of Cochrane Risk of Bias domains rated as unclear or high risk of bias) deselected. 


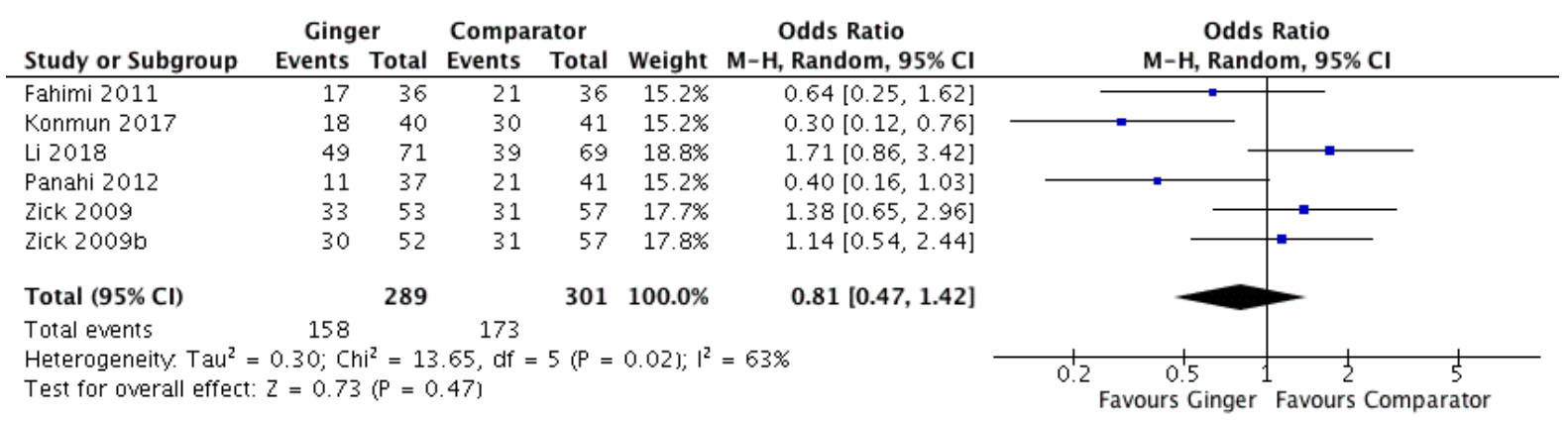

Figure 5. Ginger supplementation of any dose or duration had no association with likelihood of acute nausea (OR: $0.81,95 \%$ CI: 0.47-1.42; $\mathrm{P}=0.47 ; \mathrm{n}=5$ studies; $\mathrm{n}=6$ interventions; $\mathrm{n}=590$ participants; $I^{2}=63 \%$; GRADE level: very low). Sensitivity analysis according to dose $(\leq />1 \mathrm{~g} /$ day) or duration ( $\leq>>3$ days $)$ did not result in significant findings.

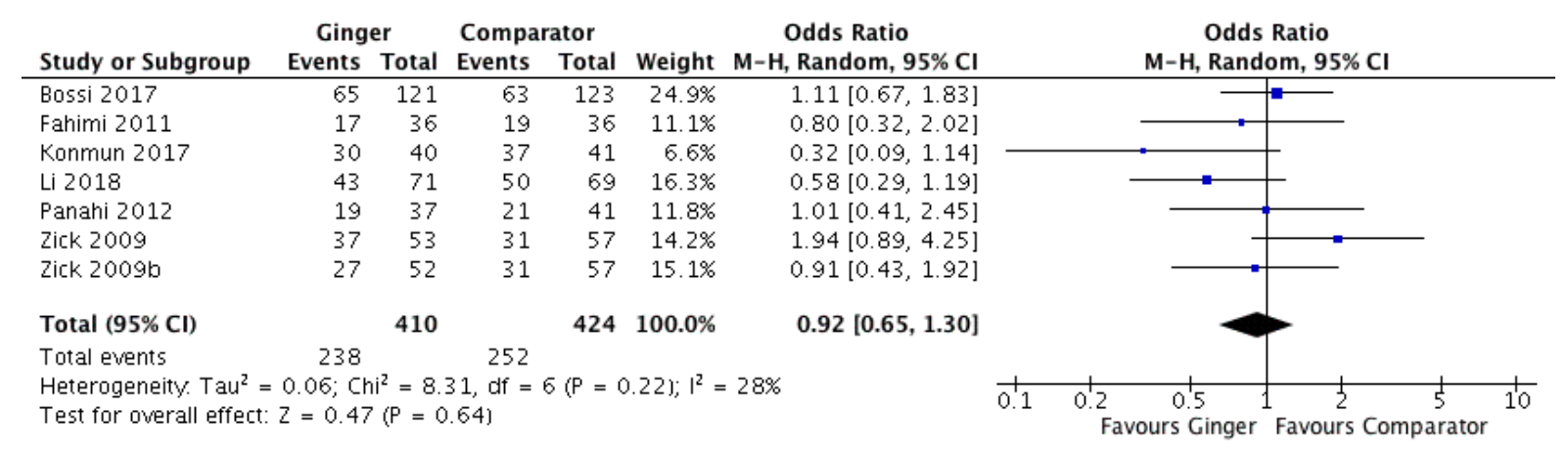

Figure 6. Ginger supplementation of any dose or duration had no association with likelihood of delayed nausea (OR: 0.92, 95\% CI: 0.65-1.30; $\mathrm{P}=0.64 ; \mathrm{n}=6$ studies; $\mathrm{n}=7$ interventions; $\mathrm{n}=834$ participants; $\mathrm{I}^{2}=28 \%$; GRADE level: moderate). Sensitivity analysis according to dose $(\leq />1 \mathrm{~g} /$ day) or duration $(\leq />3$ days $)$ did not result in significant findings. 


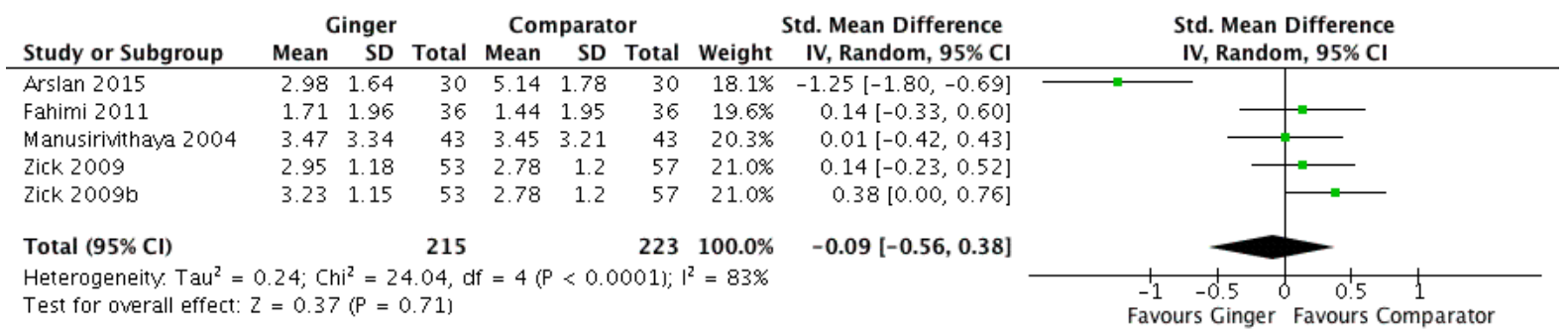

Figure 7. Ginger supplementation of any dose or duration had no association with overall nausea severity (SMD: -0.09 , 95\% CI: $-0.56-0.38 ; \mathrm{P}=0.71 ; \mathrm{n}=4$ studies; $\mathrm{n}=5$ interventions; $\mathrm{n}=438$ participants; $\mathrm{I}^{2}=83 \%$ ).

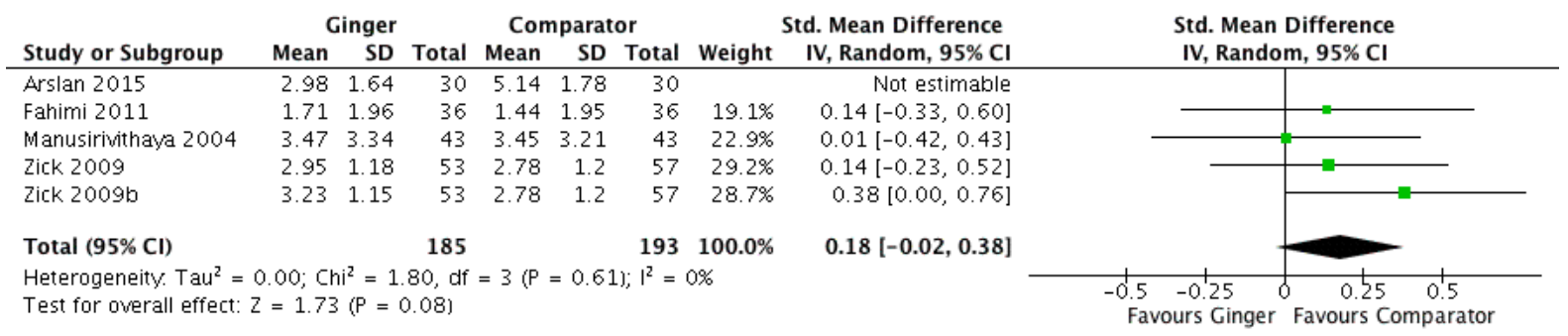

Figure 8. Ginger supplementation of any dose for any duration had no association with overall nausea severity (SMD: $0.18,95 \%$ CI: $-0.02-0.38 ; \mathrm{P}=0.08 ; \mathrm{n}=3$ studies; $\mathrm{n}=4$ interventions; $\mathrm{n}=378$ participants; $\mathrm{I}^{2}=0 \%$; GRADE level: low). Sensitivity analysis: studies with high risk of bias ( $>70 \%$ of Cochrane Risk of Bias domains rated as unclear or high risk of bias) deselected; sensitivity analysis according to dose $(\leq />1 \mathrm{~g} /$ day) or duration $(\leq />3$ days) did not result in significant findings. 


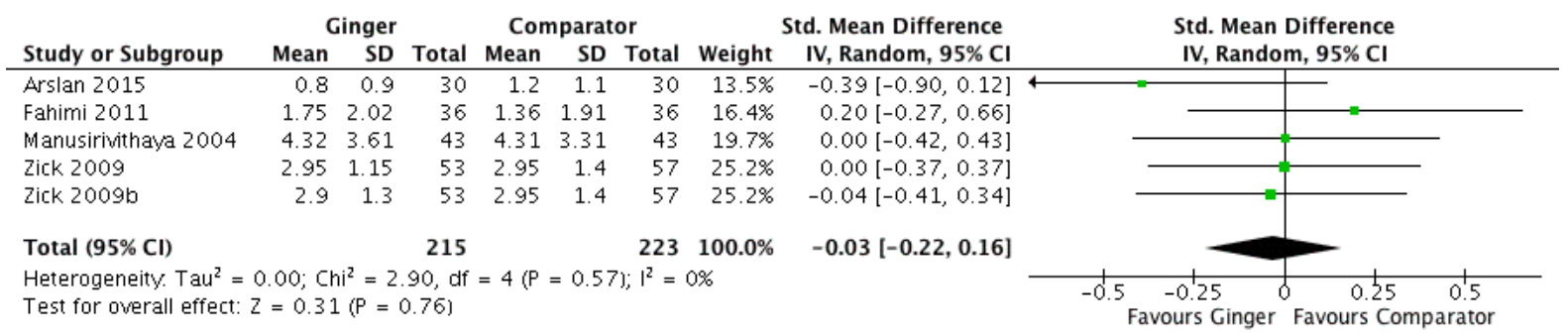

Figure 9. Ginger supplementation of any dose or duration had no association with acute nausea severity (SMD: $-0.03,95 \%$ CI: $-0.22-0.16 ; \mathrm{P}=0.76 ; \mathrm{n}=4$ studies; $\mathrm{n}=5$ interventions; $\mathrm{n}=438$ participants; $\mathrm{I}^{2}=0 \%$ ).

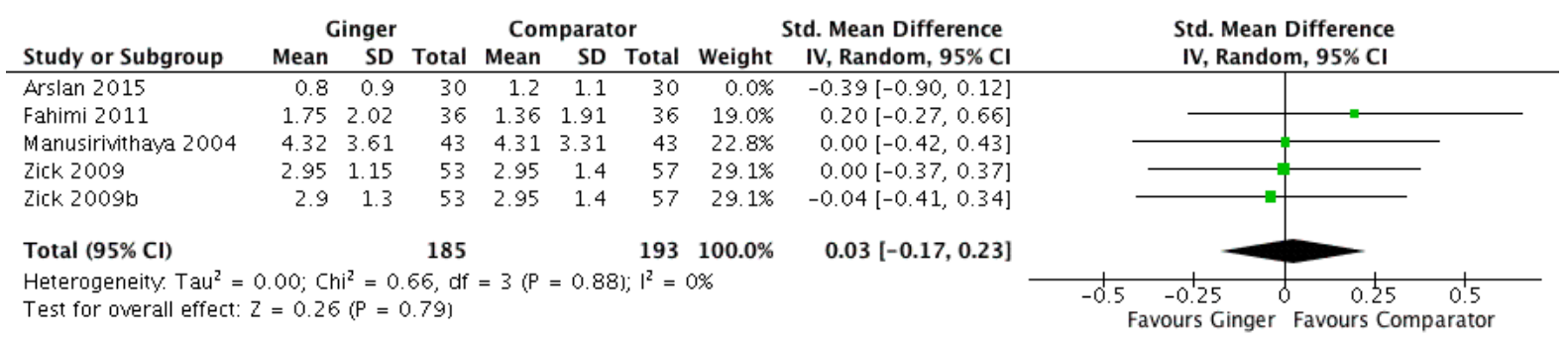

Figure 10. Ginger supplementation of any dose or duration had no association with acute nausea severity (SMD: 0.03, 95\% CI: $-0.17-0.23 ; \mathrm{P}=0.79 ; \mathrm{n}=3$ studies; $\mathrm{n}=4$ interventions; $\mathrm{n}=378$ participants; $\mathrm{I}^{2}=0 \%$; GRADE level: low). Sensitivity analysis: studies with high risk of bias ( $>70 \%$ of Cochrane Risk of Bias domains rated as unclear or high risk of bias) deselected; sensitivity analysis according to dose $(\leq />1 \mathrm{~g} /$ day) or duration $(\leq />3$ days) did not result in significant findings. 


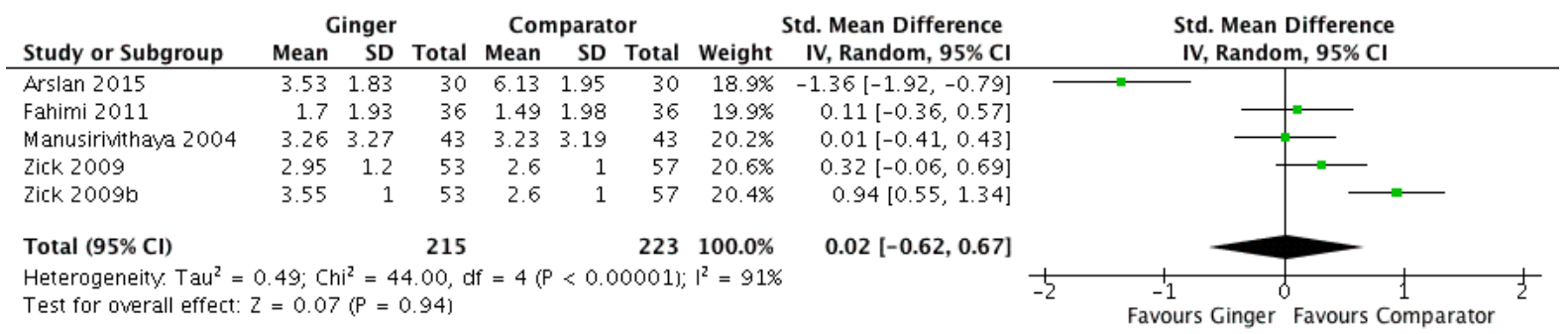

Figure 11. Ginger supplementation of any dose or duration had no association with delayed nausea severity (SMD: $0.02,95 \%$ CI: $-0.62-0.67 ; \mathrm{P}=0.94 ; \mathrm{n}=4$ studies; $\mathrm{n}=5$ interventions; $\mathrm{n}=438$ participants; $\mathrm{I}^{2}=91 \%$ ).

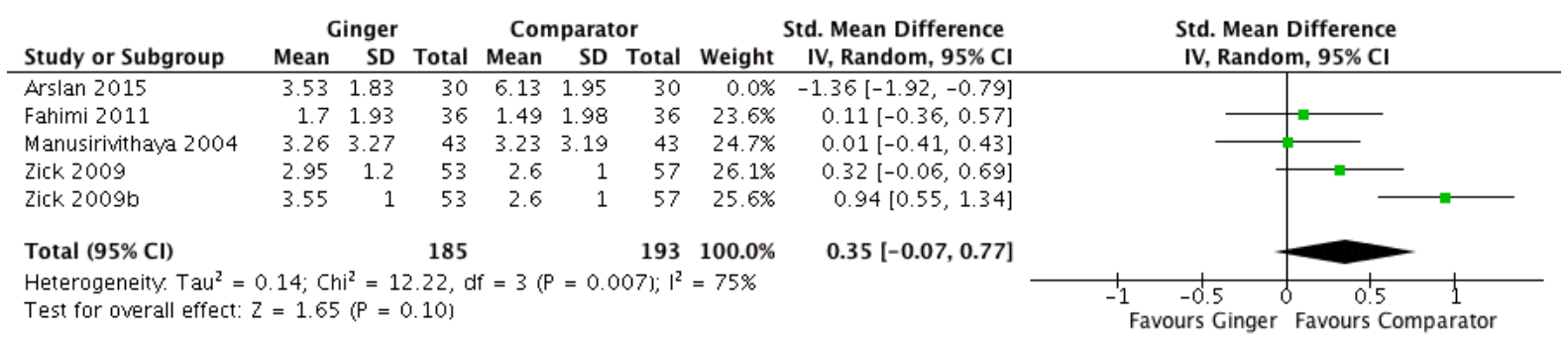

Figure 12. Ginger supplementation of any dose or duration had no association with delayed nausea severity (SMD: 0.35, 95\% CI: $-0.07-0.77 ; \mathrm{P}=0.10 ; \mathrm{n}=3$ studies; $\mathrm{n}=4$ interventions; $\mathrm{n}=378$ participants; $\mathrm{I}^{2}=75 \%$; GRADE level: very low). Sensitivity analysis: studies with high risk of bias ( $>70 \%$ of Cochrane Risk of Bias domains rated as unclear or high risk of bias) deselected. 


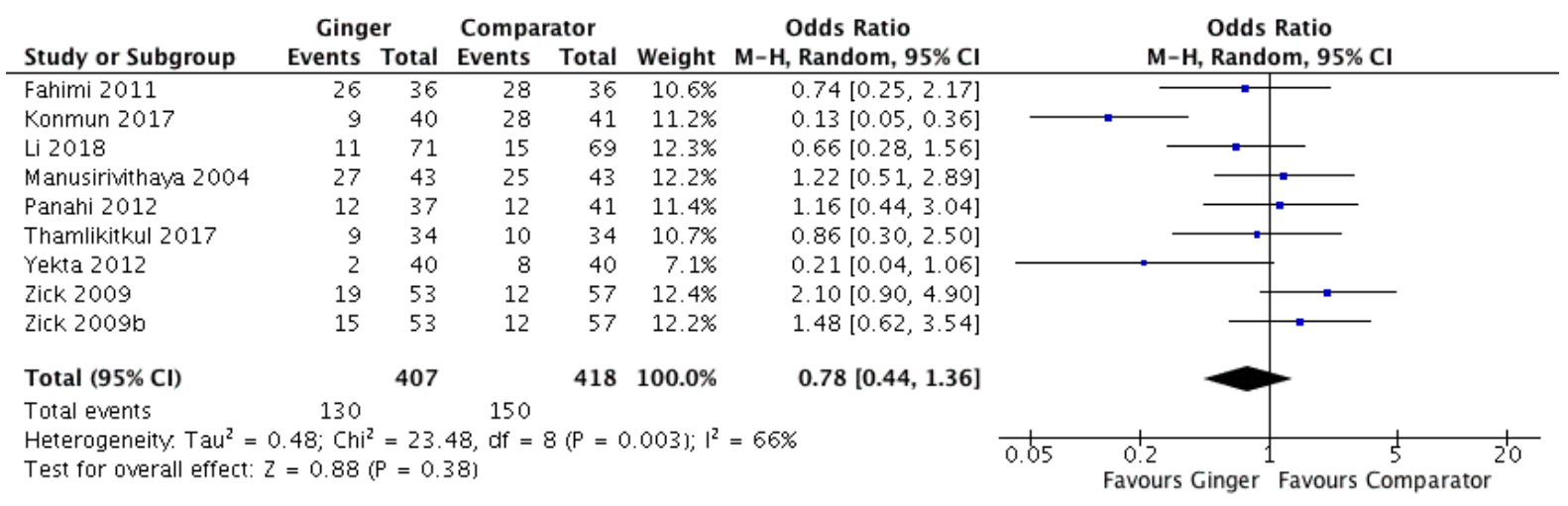

Figure 13. Ginger supplementation of any dose or duration had no association with likelihood of overall vomiting (OR: $0.78,95 \% \mathrm{CI}$ : $0.44-1.36$; $\mathrm{P}=0.38 ; \mathrm{n}=8$ studies; $\mathrm{n}=9$ interventions; $\mathrm{n}=825$ participants; $\mathrm{I}^{2}=66 \%$; GRADE level: very low). 


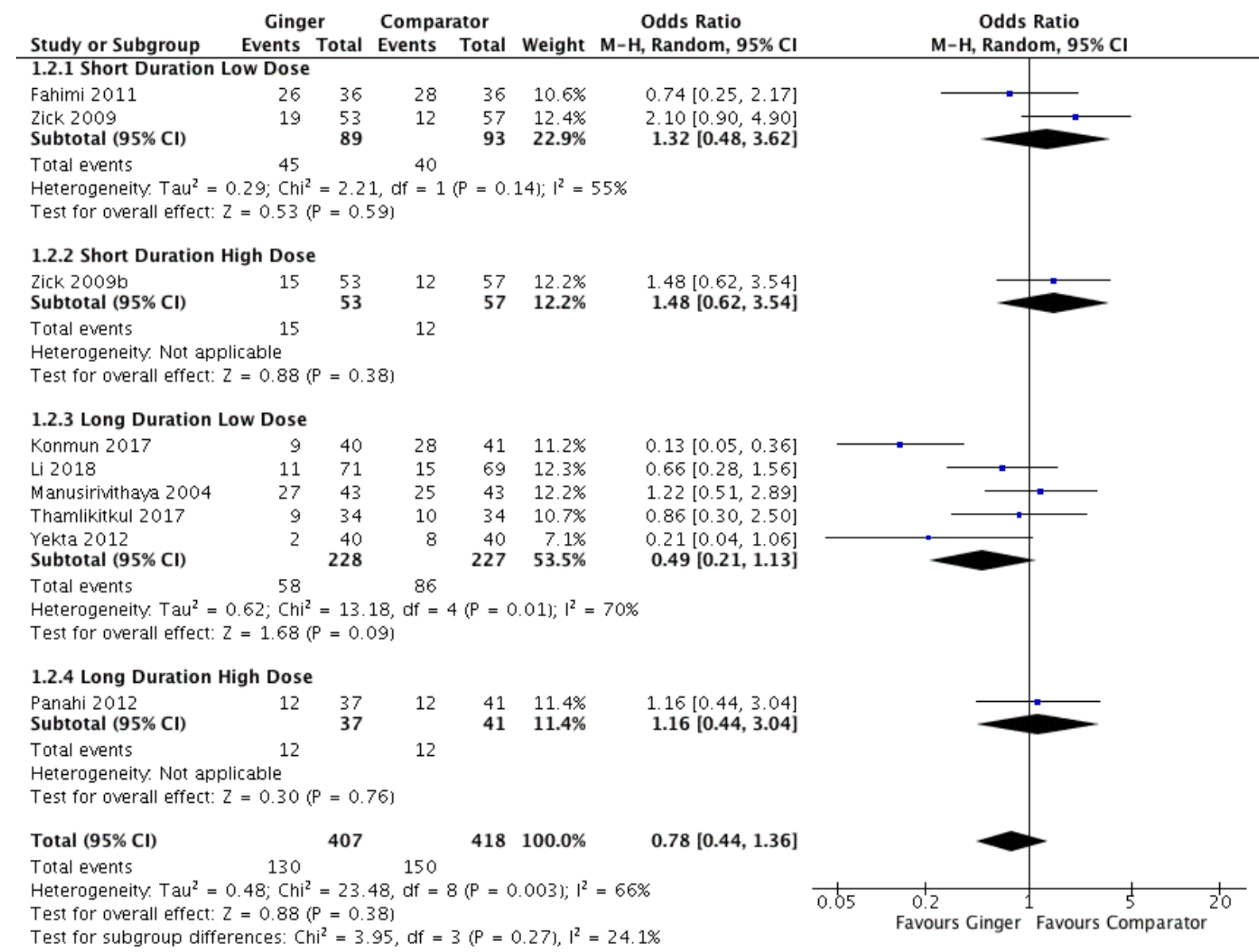

Figure 14. Ginger supplementation of varying dose and duration had no association with likelihood of overall vomiting with subgroup analysis (OR: $0.78,95 \% \mathrm{CI}$ : $0.44-1.36 ; \mathrm{P}=0.27$; $\mathrm{n}=8$ studies; $\mathrm{n}=9$ interventions; $\mathrm{n}=825$ participants; $\mathrm{I}^{2}=66 \%$ ). 


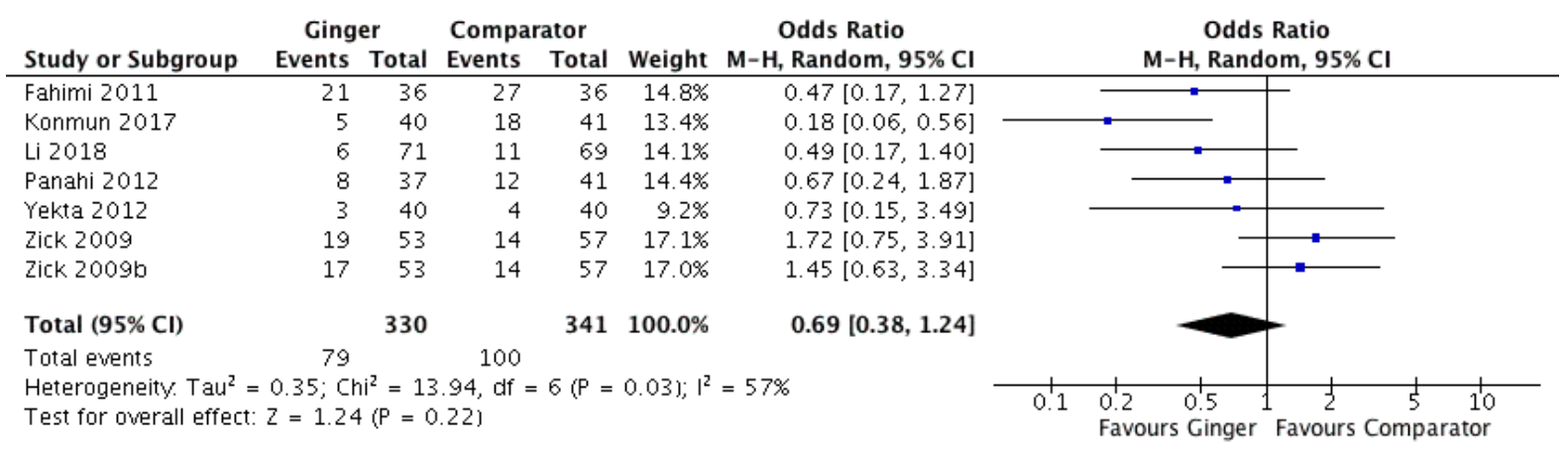

Figure 15. Ginger supplementation of any dose or duration had no association with likelihood of acute vomiting (OR: $0.69,95 \%$ CI: $0.38-1.24 ; \mathrm{P}=0.22 ; \mathrm{n}=6$ studies; $\mathrm{n}=7$ interventions; $\mathrm{n}=671$ participants; $\mathrm{I}^{2}=57 \%$; GRADE level: very low).

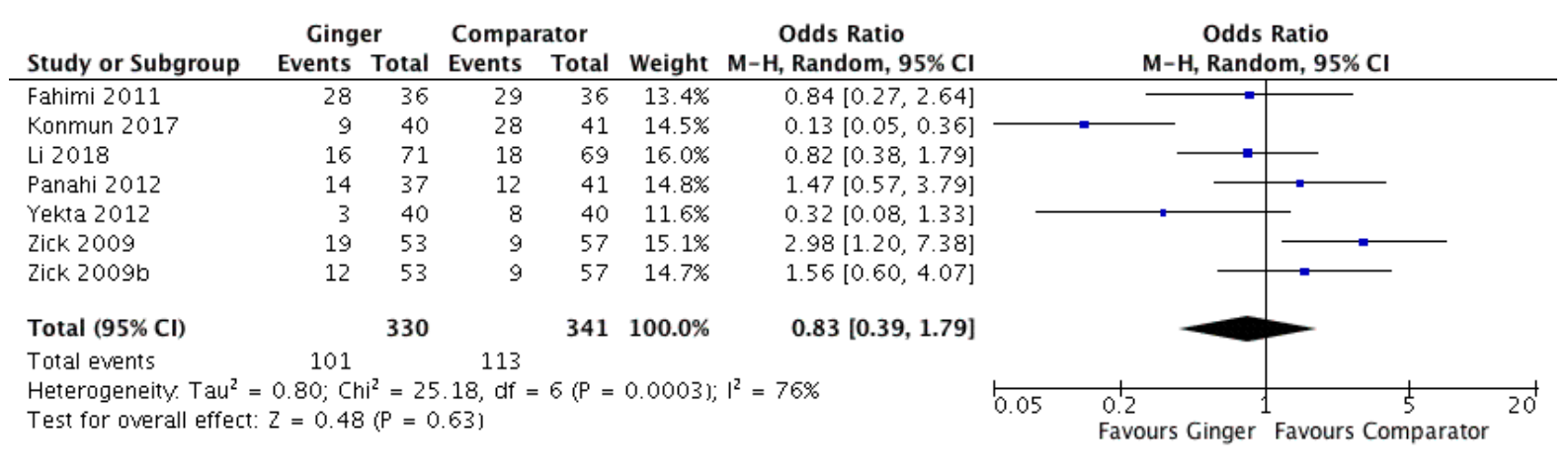

Figure 16. Ginger supplementation of any dose or duration had no association with likelihood of delayed vomiting (OR: $0.83,95 \%$ CI: $0.39-1.79 ; \mathrm{P}=0.63 ; \mathrm{n}=6$ studies; $\mathrm{n}=7$ interventions; $n=671$ participants; $I^{2}=76 \%$; GRADE level: very low). 
Ginger Comparator Odds Ratio Odds Ratio

Study or Subgroup Events Total Events Total Weight $\mathrm{M}-\mathrm{H}, \mathrm{Random}, 95 \% \mathrm{CI}$ 1.19.1 Short Duration Low Dose

Fahimi 2011

Zick 2009

Subtotal $(95 \% \mathrm{CI})$

$28 \quad 36$

29

$0.84[0.27,2.64]$

Total events

89

$93 \quad 28.5 \%$

$2.98[1.20,7.38]$

Heterogeneity. $\mathrm{Tau}^{2}=0.52 ; \mathrm{Chi}^{2}=2.88, \mathrm{df}=1(\mathrm{P}=0.09) ; \mathrm{I}^{2}=65 \%$

Test for overall effect: $Z=0.81(P=0.42)$

\subsubsection{Short Duration High Dose}

$\begin{array}{lllllll}\text { Zick } 2009 \mathrm{~b} & 12 & 53 & 9 & 57 & 14.7 \% & 1.56[0.60,4.07 \\ \text { Subtotal }(\mathbf{9 5} \% \mathrm{Cl}) & & 53 & & \mathbf{5 7} & \mathbf{1 4 . 7 \%} & \mathbf{1 . 5 6}[\mathbf{0 . 6 0}, \mathbf{4 . 0 7} \\ \text { Total events } & 12 & & 9 & & & \end{array}$

Total events

Heterogeneity. Not applicable

Test for overall effect: $Z=0.91(P=0.36)$

1.19.3 Long Duration Low Dose

Konmun 2017

Li 2018

Yekta 2012

$\begin{array}{lllll}9 & 40 & 28 & 41 & 14.5 \%\end{array}$

$\begin{array}{lllll}16 & 71 & 18 & 69 & 16.0 \%\end{array}$

Subtotal $(95 \% \mathrm{Cl})$

340

40
151

Total events $28 \quad 54$

Heterogeneity. $\mathrm{Tau}^{2}=0.82 ; \mathrm{Chi}^{2}=8.06, \mathrm{df}=2(\mathrm{P}=0.02) ; \mathrm{I}^{2}=75 \%$

Test for overall effect: $Z=1.76(P=0.08)$

\subsubsection{Long Duration High Dose}

Panahi 2012

Subtotal $(95 \% \mathrm{Cl})$

$14 \quad 37$

Total events

37
37

14

Heterogeneity. Not applicable

Test for overall effect: $Z=0.80(P=0.42)$

$\begin{array}{lllll}\text { Total }(95 \% \mathrm{Cl}) & 330 & 341 & 100.0 \% & 0.83[0.39,1.79]\end{array}$

Total events $101 \quad 113$

Heterogeneity. $\mathrm{Tau}^{2}=0.80 ; \mathrm{Chi}^{2}=25.18, \mathrm{df}=6(\mathrm{P}=0.0003) ; \mathrm{I}^{2}=76 \%$

Test for overall effect: $Z=0.48(P=0.63)$

Test for subgroup differences: $\mathrm{Chi}^{2}=4.96$, df $=3(\mathrm{P}=0.17), \mathrm{I}^{2}=39.6 \%$

$0.13[0.05,0.36]$

$0.82[0.38,1.79]$

$0.32[0.08,1.33]$

$0.34[0.10,1.13]$

M-H, Random, 95\% Cl

Figure 17. Ginger supplementation of varying dose and duration had no association with

likelihood of delayed vomiting with subgroup analysis (OR: $0.83,95 \%$ CI: 0.39-1.79; $\mathrm{P}=0.17$;

$\mathrm{n}=6$ studies; $\mathrm{n}=7$ interventions; $\mathrm{n}=671$ participants; $\mathrm{I}^{2}=76 \%$ ). 


\begin{tabular}{|c|c|c|c|c|c|c|c|c|c|c|}
\hline \multirow[b]{2}{*}{ Study or Subgroup } & \multicolumn{3}{|c|}{ Ginger } & \multicolumn{3}{|c|}{ Comparator } & \multirow[b]{2}{*}{ Weight } & \multirow{2}{*}{$\begin{array}{l}\text { Std. Mean Difference } \\
\text { IV, Random, } 95 \% \mathrm{CI}\end{array}$} & \multirow{2}{*}{\multicolumn{2}{|c|}{$\begin{array}{l}\text { Std. Mean Difference } \\
\text { IV, Random, } 95 \% \mathrm{CI}\end{array}$}} \\
\hline & Mean & SD & Total & Mean & SD & Total & & & & \\
\hline Li 2018 & 72.79 & 14 & 71 & 72.45 & 13.93 & 69 & $37.0 \%$ & $0.02[-0.31,0.36]$ & & \\
\hline Marx 2017 & 81.2 & 17.2 & 24 & 71.6 & 14.1 & 27 & $29.4 \%$ & $0.60[0.04,1.17]$ & & r \\
\hline Konmun 2017 & 86.21 & 13.6 & 46 & 72.36 & 18.9 & 42 & $33.6 \%$ & $0.84[0.40,1.28]$ & & \\
\hline Total $(95 \% \mathrm{CI})$ & & & 141 & & & 138 & $100.0 \%$ & $0.47[-0.07,1.01]$ & & \\
\hline \multicolumn{9}{|c|}{$\begin{array}{l}\text { Heterogeneity. } \mathrm{Tau}^{2}=0.18 ; \mathrm{Chi}^{2}=9.29, \mathrm{df}=2(\mathrm{P}=0.010) ; \mathrm{I}^{2}=78 \% \\
\text { Test for overall effect: } Z=1.69(\mathrm{P}=0.09)\end{array}$} & $\begin{array}{ll}-1 & -0.5 \\
\text { Favours Comparator }\end{array}$ & $\begin{array}{cc}1 & 0.5 \\
\text { Favours Ginger }\end{array}$ \\
\hline
\end{tabular}

Figure 18. Ginger supplementation of any dose for any duration had no association with quality of life (SMD: $0.47,95 \% \mathrm{CI}$ : $-0.07-1.01 ; \mathrm{P}=0.09 ; \mathrm{n}=3$ studies; $\mathrm{n}=3$ interventions; $\mathrm{I}^{2}=78 \%$; $\mathrm{n}=279$ participants; GRADE level: low).

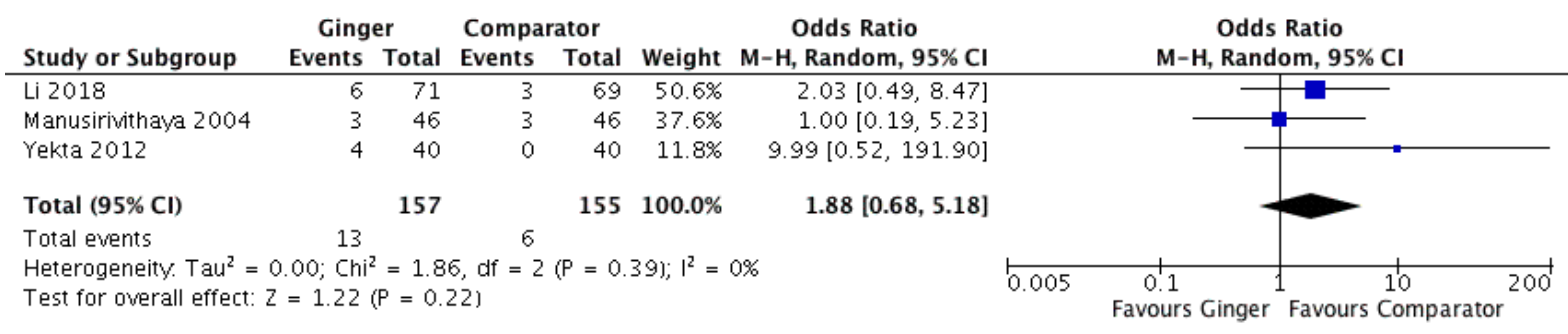

Figure 19. Ginger supplementation of any dose for any duration had no association with likelihood of heartburn (OR: $1.88,95 \%$ CI: $0.68-5.18 ; \mathrm{P}=0.22 ; \mathrm{n}=3$ studies; $\mathrm{n}=3$ interventions; $\mathrm{n}=312$ participants; $\mathrm{I}^{2}=0 \%$; GRADE level: low). 


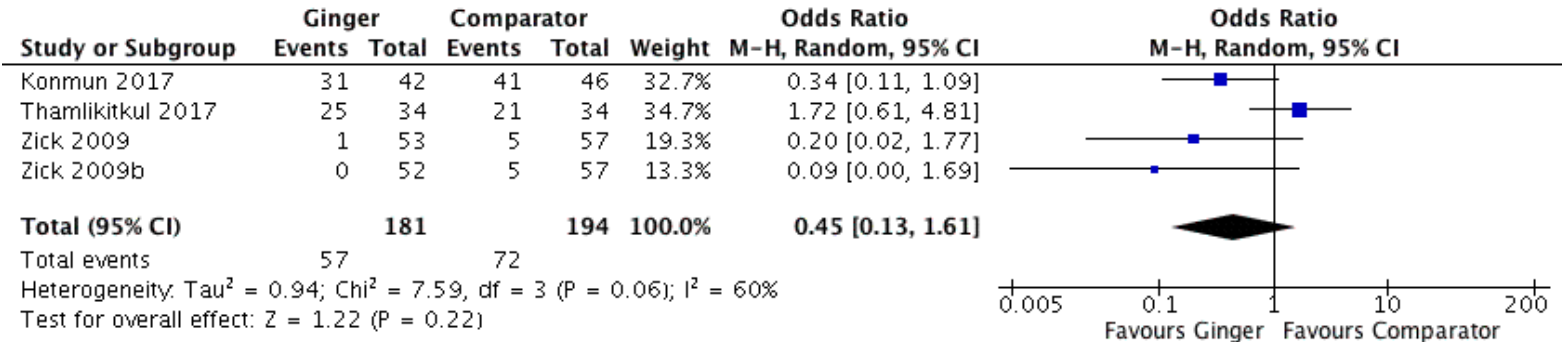

Figure 20. Ginger supplementation of any dose for any duration had no association with likelihood of fatigue (OR: $0.45,95 \%$ CI: $0.13-1.61 ; \mathrm{P}=0.22 ; \mathrm{n}=3$ studies; $\mathrm{n}=4$ interventions; $n=375$ participants; $I^{2}=60 \%$ ). 


\section{Online Supplementary Material 4: GRADE Assessment}

Table 1. GRADE assessment of ginger supplementation compared to placebo or standard care for chemotherapy-induced nausea and vomiting and related outcomes.

\begin{tabular}{|c|c|c|c|c|c|c|c|c|c|c|c|}
\hline \multicolumn{7}{|c|}{ Certainty assessment } & \multicolumn{2}{|c|}{ № of patients } & \multicolumn{2}{|c|}{ Effect } & \multirow[b]{2}{*}{ Certainty } \\
\hline № of studies & $\begin{array}{l}\text { Study } \\
\text { design }\end{array}$ & $\begin{array}{l}\text { Risk of } \\
\text { bias }\end{array}$ & Inconsistency & Indirectness & Imprecision & $\begin{array}{c}\text { Other } \\
\text { considerations }\end{array}$ & Ginger & Placebo & $\begin{array}{c}\text { Relative } \\
(95 \% \\
\text { CI })\end{array}$ & $\begin{array}{l}\text { Absolute } \\
(95 \% \text { CI) }\end{array}$ & \\
\hline \multicolumn{12}{|c|}{ Nausea Overall Incidence - Sensitivity Analysis (low risk of bias only, any duration, any dose) - Figure 2 of Online Supplementary Material 4} \\
\hline $\begin{array}{l}8 \\
\text { interventions } \\
(\mathrm{n}=7 \\
\text { studies })\end{array}$ & $\begin{array}{l}\text { randomised } \\
\text { trials }\end{array}$ & $\begin{array}{l}\text { not } \\
\text { serious }\end{array}$ & not serious & not serious & serious $^{\mathrm{d}}$ & none & $\begin{array}{l}246 / 430 \\
(57.2 \%)\end{array}$ & $\begin{array}{l}261 / 453 \\
(57.6 \%)\end{array}$ & $\begin{array}{c}\text { OR } 0.97 \\
(0.74 \text { to } \\
1.28)\end{array}$ & $\begin{array}{c}7 \text { fewer per } \\
\mathbf{1 , 0 0 0} \\
\text { (from } 75 \\
\text { fewer to } 59 \\
\text { more) }\end{array}$ & $\begin{array}{c}\oplus \oplus \oplus \bigcirc \\
\text { MODERATE }\end{array}$ \\
\hline \multicolumn{12}{|c|}{ Nausea Acute Incidence (any duration, any dose) - Figure 5 of Online Supplementary Material 4} \\
\hline $\begin{array}{l}6 \\
\text { interventions } \\
(\mathrm{n}=5 \\
\text { studies })\end{array}$ & $\begin{array}{l}\text { randomised } \\
\text { trials }\end{array}$ & $\begin{array}{l}\text { serious } \\
\mathrm{b}\end{array}$ & serious $^{c}$ & not serious & serious ${ }^{d}$ & none & $\begin{array}{l}158 / 289 \\
(54.7 \%)\end{array}$ & $\begin{array}{l}173 / 301 \\
(57.5 \%)\end{array}$ & $\begin{array}{c}\text { OR } 0.81 \\
(0.47 \text { to } \\
1.42)\end{array}$ & $\begin{array}{c}\mathbf{5 2} \text { fewer } \\
\text { per } \mathbf{1 , 0 0 0} \\
\text { (from } 186 \\
\text { fewer to } 83 \\
\text { more) }\end{array}$ & $\begin{array}{c}\oplus \bigcirc \bigcirc \bigcirc \\
\text { VERY LOW }\end{array}$ \\
\hline \multicolumn{12}{|c|}{ Nausea Delayed Incidence (any duration, any dose) - Figure 6 of Online Supplementary Material 4} \\
\hline $\begin{array}{l}7 \\
\text { interventions } \\
(n=6 \\
\text { studies })\end{array}$ & $\begin{array}{l}\text { randomised } \\
\text { trials }\end{array}$ & $\begin{array}{l}\text { not } \\
\text { serious }\end{array}$ & not serious & not serious & serious $^{\mathrm{d}}$ & none & $\begin{array}{l}238 / 410 \\
(58.0 \%)\end{array}$ & $\begin{array}{l}252 / 424 \\
(59.4 \%)\end{array}$ & $\begin{array}{c}\text { OR } 0.92 \\
(0.65 \text { to } \\
1.30)\end{array}$ & $\begin{array}{c}\mathbf{2 0} \text { fewer } \\
\text { per } \mathbf{1 , 0 0 0} \\
\text { (from } 107 \\
\text { fewer to } 61 \\
\text { more) }\end{array}$ & $\begin{array}{c}\oplus \oplus \oplus \bigcirc \\
\text { MODERATE }\end{array}$ \\
\hline
\end{tabular}




\begin{tabular}{|c|c|c|c|c|c|c|c|c|c|c|c|}
\hline \multicolumn{7}{|c|}{ Certainty assessment } & \multicolumn{2}{|c|}{ № of patients } & \multicolumn{2}{|c|}{ Effect } & \multirow[b]{2}{*}{ Certainty } \\
\hline № of studies & $\begin{array}{l}\text { Study } \\
\text { design }\end{array}$ & $\begin{array}{c}\text { Risk of } \\
\text { bias }\end{array}$ & Inconsistency & Indirectness & Imprecision & $\begin{array}{c}\text { Other } \\
\text { considerations }\end{array}$ & Ginger & Placebo & $\begin{array}{c}\text { Relative } \\
\text { (95\% } \\
\text { CI) }\end{array}$ & $\begin{array}{l}\text { Absolute } \\
\text { (95\% CI) }\end{array}$ & \\
\hline $\begin{array}{l}4 \\
\text { interventions } \\
(\mathrm{n}=3 \\
\text { studies })\end{array}$ & $\begin{array}{l}\text { randomised } \\
\text { trials }\end{array}$ & $\begin{array}{l}\text { not } \\
\text { serious }\end{array}$ & not serious & not serious & $\begin{array}{l}\text { very serious } \\
\text { a }\end{array}$ & none & 142 & 150 & - & $\begin{array}{c}\text { SMD 0.18 } \\
\text { higher } \\
(0.02 \text { lower } \\
\text { to } 0.38 \\
\text { higher })\end{array}$ & $\begin{array}{c}\oplus \oplus \bigcirc \bigcirc \\
\text { LOW }\end{array}$ \\
\hline \multicolumn{12}{|c|}{ Nausea Acute Severity - Sensitivity Analysis (low risk of bias only, any duration, any dose) - Figure 10 of Online Supplementary Material 4} \\
\hline $\begin{array}{l}4 \\
\text { interventions } \\
(\mathrm{n}=3 \\
\text { studies })\end{array}$ & $\begin{array}{l}\text { randomised } \\
\text { trials }\end{array}$ & $\begin{array}{l}\text { not } \\
\text { serious }\end{array}$ & not serious & not serious & $\begin{array}{l}\text { very serious } \\
\text { a }\end{array}$ & none & 185 & 193 & - & $\begin{array}{c}\text { SMD 0.03 } \\
\text { SD higher } \\
\text { (0.17 lower } \\
\text { to } 0.23 \\
\text { higher) }\end{array}$ & $\begin{array}{c}\oplus \oplus \bigcirc \bigcirc \\
\text { LOW }\end{array}$ \\
\hline \multicolumn{12}{|c|}{ Nausea Delayed Severity - Sensitivity Analysis (low risk of bias only, any duration, any dose) - Figure 12 of Online Supplementary Material 4} \\
\hline $\begin{array}{l}4 \\
\text { interventions } \\
(\mathrm{n}=3 \\
\text { studies })\end{array}$ & $\begin{array}{l}\text { randomised } \\
\text { trials }\end{array}$ & $\begin{array}{l}\text { not } \\
\text { serious }\end{array}$ & serious $^{\mathrm{c}}$ & not serious & $\begin{array}{l}\text { very serious } \\
\text { a }\end{array}$ & none & 185 & 193 & - & $\begin{array}{c}\text { SMD 0.35 } \\
\text { SD higher } \\
\text { (0.07 lower } \\
\text { to } 0.77 \\
\text { higher })\end{array}$ & $\begin{array}{c}\oplus \bigcirc \bigcirc \bigcirc \\
\text { VERY LOW }\end{array}$ \\
\hline \multicolumn{12}{|c|}{ Vomiting Overall Incidence (any duration, any dose) - Figure 13 of Online Supplementary Material 4} \\
\hline $\begin{array}{l}9 \\
\text { interventions } \\
(\mathrm{n}=8 \\
\text { studies })\end{array}$ & $\begin{array}{l}\text { randomised } \\
\text { trials }\end{array}$ & $\begin{array}{l}\text { not } \\
\text { serious }\end{array}$ & serious $^{\mathrm{c}}$ & not serious & $\begin{array}{l}\text { very serious } \\
d\end{array}$ & none & $\begin{array}{l}130 / 407 \\
(31.9 \%)\end{array}$ & $\begin{array}{l}150 / 418 \\
(35.9 \%)\end{array}$ & $\begin{array}{c}\text { OR 0.78 } \\
(0.44 \text { to } \\
1.36)\end{array}$ & $\begin{array}{c}\mathbf{5 5} \text { fewer } \\
\text { per } \mathbf{1 , 0 0 0} \\
\text { (from } 163 \\
\text { fewer to } 74 \\
\text { more) }\end{array}$ & $\begin{array}{c}\oplus \bigcirc \bigcirc \bigcirc \\
\text { VERY LOW }\end{array}$ \\
\hline
\end{tabular}




\begin{tabular}{|c|c|c|c|c|c|c|c|c|c|c|c|}
\hline \multicolumn{7}{|c|}{ Certainty assessment } & \multicolumn{2}{|c|}{ № of patients } & \multicolumn{2}{|c|}{ Effect } & \multirow[b]{2}{*}{ Certainty } \\
\hline № of studies & $\begin{array}{l}\text { Study } \\
\text { design }\end{array}$ & $\begin{array}{c}\text { Risk of } \\
\text { bias }\end{array}$ & Inconsistency & Indirectness & Imprecision & $\begin{array}{c}\text { Other } \\
\text { considerations }\end{array}$ & Ginger & Placebo & $\begin{array}{c}\text { Relative } \\
(95 \% \\
\text { CI) }\end{array}$ & $\begin{array}{l}\text { Absolute } \\
(95 \% \text { CI) }\end{array}$ & \\
\hline $\begin{array}{l}7 \\
\text { interventions } \\
(\mathrm{n}=6 \\
\text { studies })\end{array}$ & $\begin{array}{l}\text { randomised } \\
\text { trials }\end{array}$ & $\begin{array}{l}\text { serious } \\
\mathrm{q}\end{array}$ & serious ${ }^{\mathrm{e}}$ & not serious & $\begin{array}{l}\text { very serious } \\
\text { d }\end{array}$ & none & $\begin{array}{c}79 / 330 \\
(23.9 \%)\end{array}$ & $\begin{array}{l}100 / 341 \\
(29.3 \%)\end{array}$ & $\begin{array}{c}\text { OR 0.69 } \\
(0.38 \text { to } \\
1.24)\end{array}$ & $\begin{array}{c}\mathbf{7 1} \text { fewer } \\
\text { per } \mathbf{1 , 0 0 0} \\
\text { (from } 157 \\
\text { fewer to } 47 \\
\text { more) }\end{array}$ & $\begin{array}{c}\oplus \bigcirc \bigcirc \bigcirc \\
\text { VERY LOW }\end{array}$ \\
\hline \multicolumn{12}{|c|}{ Vomiting Acute Incidence - Subgroup Analysis ( $>3$-days duration, $\leq 1 \mathrm{~g} /$ day) - Figure 3 of Manuscript } \\
\hline $\begin{array}{l}3 \\
\text { interventions } \\
(\mathrm{n}=3 \\
\text { studies })\end{array}$ & $\begin{array}{l}\text { randomised } \\
\text { trials }\end{array}$ & $\begin{array}{l}\text { not } \\
\text { serious }\end{array}$ & not serious & not serious & serious $^{\text {a }}$ & none & $\begin{array}{l}14 / 151 \\
(9.3 \%)\end{array}$ & $\begin{array}{c}33 / 150 \\
(22.0 \%)\end{array}$ & $\begin{array}{c}\text { OR } 0.37 \\
(0.17 \text { to } \\
0.81)\end{array}$ & $\begin{array}{l}126 \text { fewer } \\
\text { per } \mathbf{1 , 0 0 0} \\
\text { (from } 174 \\
\text { fewer to } 34 \\
\text { fewer) }\end{array}$ & $\begin{array}{c}\oplus \oplus \oplus \bigcirc \\
\text { MODERATE }\end{array}$ \\
\hline \multicolumn{12}{|c|}{ Vomiting Delayed Incidence (any duration, any dose) - Figure 16 of Online Supplementary Material 4} \\
\hline $\begin{array}{l}7 \\
\text { interventions } \\
(\mathrm{n}=6 \\
\text { studies })\end{array}$ & $\begin{array}{l}\text { randomised } \\
\text { trials }\end{array}$ & $\begin{array}{l}\text { serious } \\
\mathrm{s}\end{array}$ & serious ${ }^{\mathrm{e}}$ & not serious & serious ${ }^{a}$ & none & $\begin{array}{l}101 / 330 \\
(30.6 \%)\end{array}$ & $\begin{array}{l}113 / 341 \\
(33.1 \%)\end{array}$ & $\begin{array}{c}\text { OR } \mathbf{0 . 8 3} \\
(0.39 \text { to } \\
1.79)\end{array}$ & $\begin{array}{c}1 \text { fewer per } \\
\mathbf{1 , 0 0 0} \\
\text { (from } 2 \\
\text { fewer to } 0 \\
\text { fewer) }\end{array}$ & $\begin{array}{c}\oplus \bigcirc \bigcirc \bigcirc \\
\text { VERY LOW }\end{array}$ \\
\hline \multicolumn{12}{|c|}{ Quality of Life Score (any duration, any dose) - Figure 18 of Online Supplementary Material 4} \\
\hline $\begin{array}{l}3 \\
\text { interventions } \\
(\mathrm{n}=3 \\
\text { studies })\end{array}$ & $\begin{array}{l}\text { randomised } \\
\text { trials }\end{array}$ & $\begin{array}{l}\text { not } \\
\text { serious }\end{array}$ & serious $^{c}$ & not serious & serious $^{a}$ & none & 117 & 111 & - & $\begin{array}{l}\text { SMD 0.47 } \\
\text { SD higher } \\
(0.07 \text { lower } \\
\text { to } 1.01 \\
\text { higher })\end{array}$ & $\begin{array}{l}\oplus \oplus \bigcirc \bigcirc \\
\text { LOW }\end{array}$ \\
\hline
\end{tabular}




\begin{tabular}{|c|c|c|c|c|c|c|c|c|c|c|c|}
\hline \multicolumn{7}{|c|}{ Certainty assessment } & \multicolumn{2}{|c|}{ № of patients } & \multicolumn{2}{|c|}{ Effect } & \multirow[b]{2}{*}{ Certainty } \\
\hline № of studies & $\begin{array}{l}\text { Study } \\
\text { design }\end{array}$ & $\begin{array}{c}\text { Risk of } \\
\text { bias }\end{array}$ & Inconsistency & Indirectness & Imprecision & $\begin{array}{c}\text { Other } \\
\text { considerations }\end{array}$ & Ginger & Placebo & $\begin{array}{c}\text { Relative } \\
\text { (95\% } \\
\text { CI) }\end{array}$ & $\begin{array}{l}\text { Absolute } \\
\text { (95\% CI) }\end{array}$ & \\
\hline $\begin{array}{l}2 \\
\text { interventions } \\
\text { ( } \mathrm{n}=1 \text { study) }\end{array}$ & $\begin{array}{l}\text { randomised } \\
\text { trials }\end{array}$ & $\begin{array}{l}\text { not } \\
\text { serious }\end{array}$ & not serious & not serious & $\begin{array}{l}\text { very serious } \\
\text { a }\end{array}$ & none & $\begin{array}{l}1 / 105 \\
(1.0 \%)\end{array}$ & $\begin{array}{l}10 / 114 \\
(8.8 \%)\end{array}$ & $\begin{array}{c}\text { OR 0.15 } \\
(0.03 \text { to } \\
0.87)\end{array}$ & $\begin{array}{c}74 \text { fewer } \\
\text { per } \mathbf{1 , 0 0 0} \\
\text { (from } 85 \\
\text { fewer to } 65 \\
\text { more) }\end{array}$ & $\begin{array}{c}\oplus \oplus \bigcirc \bigcirc \\
\text { LOW }\end{array}$ \\
\hline \multicolumn{12}{|c|}{ Any Adverse Effects Incidence (any duration, any dose) - Figure 5 of Manuscript } \\
\hline $\begin{array}{l}5 \\
\text { interventions } \\
(\mathrm{n}=3 \\
\text { studies })\end{array}$ & $\begin{array}{l}\text { randomised } \\
\text { trials }\end{array}$ & $\begin{array}{l}\text { not } \\
\text { serious }\end{array}$ & not serious & not serious & serious $^{\text {a }}$ & none & $\begin{array}{l}96 / 725 \\
(13.2 \%)\end{array}$ & $\begin{array}{l}58 / 733 \\
(7.9 \%)\end{array}$ & $\begin{array}{c}\text { OR 2.03 } \\
(1.39 \text { to } \\
2.99)\end{array}$ & $\begin{array}{c}\mathbf{6 9} \text { more } \\
\text { per } 1,000 \\
\text { (from } 28 \\
\text { more to } 125 \\
\text { more) }\end{array}$ & $\begin{array}{c}\oplus \oplus \oplus \bigcirc \\
\text { MODERATE }\end{array}$ \\
\hline \multicolumn{12}{|c|}{ Heartburn Incidence (any duration, any dose) - Figure 19 of Online Supplementary Material 4} \\
\hline $\begin{array}{l}3 \\
\text { interventions } \\
(\mathrm{n}=3 \\
\text { studies })\end{array}$ & $\begin{array}{l}\text { randomised } \\
\text { trials }\end{array}$ & $\begin{array}{l}\text { not } \\
\text { serious }\end{array}$ & not serious & not serious & $\begin{array}{l}\text { very serious } \\
\text { a }\end{array}$ & none & $\begin{array}{l}13 / 157 \\
(8.3 \%)\end{array}$ & $\begin{array}{l}6 / 155 \\
(3.9 \%)\end{array}$ & $\begin{array}{c}\text { OR 1.88 } \\
(0.68 \text { to } \\
5.18)\end{array}$ & $\begin{array}{c}32 \text { more } \\
\text { per } \mathbf{1 , 0 0 0} \\
\text { (from } 12 \\
\text { fewer to } \\
134 \text { more) }\end{array}$ & $\begin{array}{c}\oplus \oplus \bigcirc \bigcirc \\
\text { LOW }\end{array}$ \\
\hline
\end{tabular}

CI: Confidence interval; OR: Odds ratio; SMD: Standardised mean difference

Explanations

a. Wide CI; small sample size and small number of events.

b. 2/6 studies rated 'unclear' and 1/6 'high' for random sequence generation bias; 4/6 'unclear' and 1/6 'high' for allocation concealment bias.

c. Large variation in effect size; minimal overlap in confidence intervals; I2 high; $\mathrm{P}<0.05$.

d. Moderately wide CI's

e. 5/5 studies rated 'unclear' risk of bias and 1/5 'high' for allocation concealment, however, unlikely to largely affect outcomes.

f. 1/4 studies rated 'unclear' and 1/4 studies rated 'high' for random sequence generation bias; 2/4 rated 'unclear' and 1/4 rated 'high' for allocation concealment bias. 
g. 1/4 studies rated 'unclear' and 1/4 'high' for random sequence generation bias; 3/4 'unclear' and 1/4 'high' for allocation concealment bias. 
